REPRESENTATION THEORY

An Electronic Journal of the American Mathematical Society

Volume 12, Pages 131-169 (March 10, 2008)

S 1088-4165(08)00325-7

\title{
GEOMETRIC BRAID GROUP ACTION ON DERIVED CATEGORIES OF COHERENT SHEAVES
}

\author{
SIMON RICHE, \\ WITH A JOINT APPENDIX WITH ROMAN BEZRUKAVNIKOV
}

\begin{abstract}
In this paper we give, for semi-simple groups without factors of type $\mathbf{G}_{2}$, a geometric construction of a braid group action on $\mathcal{D}^{b} \operatorname{Coh}(\widetilde{\mathfrak{g}})$ extending the action constructed by Bezrukavnikov, Mirković and Rumynin in the context of localization in positive characteristic. It follows that this action extends to characteristic zero, where it also has some nice representationtheoretic interpretations. The argument uses a presentation of the affine braid group analogous to the "Bernstein presentation" of the corresponding Hecke algebra (this presentation was suggested by Lusztig; it is worked out in the appendix, written jointly with Roman Bezrukavnikov).
\end{abstract}

\section{INTRODUCTION}

0.1. Let $G$ be a connected, semi-simple, simply-connected algebraic group over an algebraically closed field $\mathbb{k}$, and let $\mathfrak{g}=\operatorname{Lie}(G)$. In BMR2], Bezrukavnikov, Mirković and Rumynin have constructed an action of the extended affine braid group associated to $G$ on the category $\mathcal{D}^{b} \operatorname{Coh}_{\mathcal{B}_{\chi}^{(1)}}\left(\widetilde{\mathfrak{g}}^{(1)}\right)$, when $\operatorname{char}(\mathbb{k})$ is greater than the Coxeter number $h$ of $G$ (here $\chi \in \mathfrak{g}^{*}$ is nilpotent, and $\mathcal{B}_{\chi}$ is the corresponding Springer fiber). Their construction relies on deep results relating the modules over $\mathcal{U} \mathfrak{g}$ (the enveloping algebra of $\mathfrak{g}$ ), $\mathcal{D}$-modules on the flag variety of $G$, and coherent sheaves on $\widetilde{\mathfrak{g}}^{(1)}$. In this paper we show that, when $G$ has no factor of type $\mathbf{G}_{2}$, this action can be defined geometrically, without any reference to representation theory. In particular, we obtain that the action can also be defined when $\operatorname{char}(\mathbb{k}) \leq h$ (except for $\operatorname{char}(\mathbb{k})=2$ in the non-simply-laced case), including characteristic 0 . We also obtain that similar actions can be defined on various other categories, such as $\mathcal{D}^{b} \operatorname{Coh}(\widetilde{\mathfrak{g}}), \mathcal{D}^{b} \operatorname{Coh}(\widetilde{\mathcal{N}}), \mathcal{D}^{b} \operatorname{Coh}^{G}(\widetilde{\mathfrak{g}})$ or $\mathcal{D}^{b} \operatorname{Coh}^{G}(\widetilde{\mathcal{N}})$. For $\mathbb{k}=\mathbb{C}$, this action is related to Ginzburg's interpretation of the equivariant K-theory of the Steinberg variety, and to Springer representations of the finite Weyl group on the homology of Springer fibers.

0.2. More precisely, let $G$ be a connected, semi-simple, simply-connected algebraic group over $\mathbb{k}$, let $T$ be a maximal torus of $G, \mathbb{X}$ the character group of $T, R$ the root system of $(G, T), W$ its Weyl group, and $\Phi$ a basis of $R$. The extended affine Weyl group $W_{\text {aff }}^{\prime}:=W \ltimes \mathbb{X}$ has a natural "length function" $\ell$, although it is not a Coxeter group in general (see 1.1). The extended affine braid group $B_{\text {aff }}^{\prime}$ is by

Received by the editors March 12, 2007 and, in revised form, July 23, 2007.

2000 Mathematics Subject Classification. Primary 14M15; Secondary 20F55, 18 E30.

(C)2008 American Mathematical Society 
definition the group with presentation:

$$
\begin{array}{ll}
\text { Generators: } & T_{w}\left(w \in W_{\text {aff }}^{\prime}\right) ; \\
\text { Relations: } & T_{v} T_{w}=T_{v w} \text { if } \ell(v w)=\ell(v)+\ell(w) .
\end{array}
$$

This definition is similar to the "Iwahori-Matsumoto presentation" of the corresponding Hecke algebra $\mathcal{H}$. If $x \in \mathbb{X}$, write $x=x_{1}-x_{2}$ with $x_{1}, x_{2}$ dominant weights. Then $\theta_{x}:=T_{x_{1}}\left(T_{x_{2}}\right)^{-1}$ depends only on $x$. If $\alpha, \beta \in \Phi$, we denote by $n_{\alpha, \beta}$ the order of $s_{\alpha} s_{\beta}$ in $W$. Our first step, obtained as a joint work with Roman Bezrukavnikor 1 (see the Appendix), is a second presentation of $B_{\mathrm{aff}}^{\prime}$, which is an analogue of the "Bernstein presentation" of $\mathcal{H}$. The idea of this presentation is due to Lusztig (see e.g. L2]). It is given by:

Generators: $T_{s_{\alpha}}(\alpha \in \Phi), \theta_{x}(x \in \mathbb{X})$;

Relations: $\quad$ (1) $T_{s_{\alpha}} T_{s_{\beta}} \cdots=T_{s_{\beta}} T_{s_{\alpha}} \cdots\left(n_{\alpha, \beta}\right.$ elements on each side);

(2) $\theta_{x} \theta_{y}=\theta_{x+y}$;

(3) $T_{s_{\alpha}} \theta_{x}=\theta_{x} T_{s_{\alpha}}$ if $\left\langle x, \alpha^{\vee}\right\rangle=0$;

(4) $\theta_{x}=T_{s_{\alpha}} \theta_{x-\alpha} T_{s_{\alpha}}$ if $\left\langle x, \alpha^{\vee}\right\rangle=1$.

Let $\mathfrak{g}=\operatorname{Lie}(G)$. Let $B$ be the Borel subgroup of $G$ containing $T$ such that the roots of $\mathfrak{g} / \mathfrak{b}$ are the positive ones, where $\mathfrak{b}$ is the Lie algebra of $B$. Let $\mathfrak{n}$ be the nilpotent radical of $\mathfrak{b}$. Let $\widetilde{\mathcal{N}}:=T^{*}(G / B)$ be the cotangent bundle of $G / B$, and $\widetilde{\mathfrak{g}}$ be the "extended" cotangent bundle

$$
\widetilde{\mathfrak{g}}:=\left\{(X, g B) \in \mathfrak{g}^{*} \times G / B \mid X_{\mid g \cdot \mathfrak{n}}=0\right\} .
$$

Our main result is the construction of a weak action of $B_{\text {aff }}^{\prime}$ on the category $\mathcal{D}^{b} \operatorname{Coh}(\widetilde{\mathfrak{g}})$, by convolution. Using the preceding presentation, to construct this action it is sufficient to define kernels associated to the generators $T_{s_{\alpha}}$ and $\theta_{x}$, and to verify relations (1) to (4) for these kernels. The kernel associated with $T_{s_{\alpha}}$ is $\mathcal{O}_{S_{\alpha}}$ for some closed subvariety $S_{\alpha} \subset \widetilde{\mathfrak{g}} \times \widetilde{\mathfrak{g}}$ (see 1.4 for a precise definition), and the kernel associated with $\theta_{x}$ is $\Delta_{*} \mathcal{O}_{\mathfrak{\mathfrak { g }}}(x)$ where $\Delta: \widetilde{\mathfrak{g}} \hookrightarrow \widetilde{\mathfrak{g}} \times \widetilde{\mathfrak{g}}$ is the diagonal embedding. Relations (2), (3) and (4) for these kernels are easy to prove.

The most difficult relations to prove are the "finite braid relations", i.e., relations (1). For this proof we have to assume that $G$ has no factor of type $\mathbf{G}_{2}$, and to perform a case-by-case analysis, depending on whether $\alpha$ and $\beta$ generate a root system of type $\mathbf{A}_{1} \times \mathbf{A}_{1}, \mathbf{A}_{2}$ or $\mathbf{B}_{2}$ (see sections 2 and 3 ). Our proof involves the study of Demazure-like "resolutions" $3 \widetilde{\mathcal{Z}}_{\left(s_{1}, s_{2}, \cdots, s_{n}\right)} \rightarrow S_{w}$. Here $w$ is the element of $W$ corresponding to the finite braid relation under consideration, $S_{w}$ is a vector fibration over the $G$-orbit closure $\mathcal{X}_{w} \subset(G / B) \times(G / B)$ associated with $w$, and $\widetilde{\mathcal{Z}}_{\left(s_{1}, s_{2}, \cdots, s_{n}\right)}$ is a vector fibration over the Demazure resolution of $\mathcal{X}_{w}$ associated with the reduced decomposition $w=s_{1} s_{2} \cdots s_{n}$.

Finally, we obtain (see Theorem 1.4.1) that if $G$ has no factor of type $\mathbf{G}_{2}$, and $\operatorname{char}(\mathbb{k}) \neq 2$ if $R$ is not simply-laced, there exists an action of $B_{\text {aff }}^{\prime}$ on $\mathcal{D}^{b} \operatorname{Coh}(\widetilde{\mathfrak{g}})$ such that:

(i) The action of $\theta_{x}$ is given by the convolution with kernel $\Delta_{*} \mathcal{O}_{\mathfrak{\mathfrak { g }}}(x)$;

(ii) The action of $T_{s_{\alpha}}$ is given by the convolution with kernel $\mathcal{O}_{S_{\alpha}}$.

\footnotetext{
${ }^{1}$ After this paper was submitted, Valerio Toledano Laredo pointed out to us that this presentation is also proved in Macdonald's book [M]. Our proof is different.

${ }^{2} \mathrm{See} 1.3$

${ }^{3}$ These are not really resolutions of singularities, as the variety $\widetilde{\mathcal{Z}}_{\left(s_{1}, s_{2}, \cdots, s_{n}\right)}$ is singular. But we show that they share some properties with resolutions of singularities.
} 
We expect this result to hold also when $G$ has a factor of type $\mathbf{G}_{2}$. We plan to come back to this question in a future publication, possibly following a less computational approach.

The proof of this result occupies sections 1.2 to 3 . In the remaining three sections we study the compatibility of this action with the inclusion $\widetilde{\mathcal{N}} \hookrightarrow \widetilde{\mathfrak{g}}$, and with some representation-theoretic constructions.

First, in section 4 we show that one can similarly define an action of $B_{\mathrm{aff}}^{\prime}$ on the category $\mathcal{D}^{b} \operatorname{Coh}(\widetilde{\mathcal{N}})$, such that the following diagram is commutative for any $b \in B_{\text {aff }}^{\prime}$, where $i: \widetilde{\mathcal{N}} \hookrightarrow \widetilde{\mathfrak{g}}$ denotes the natural embedding:

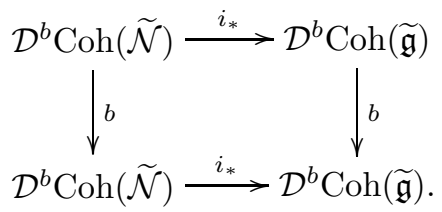

In section $\left[5\right.$ we show that the action of $B_{\text {aff }}^{\prime}$ on $\mathcal{D}^{b} \operatorname{Coh}(\mathfrak{g})$, or rather the similar action on $\mathcal{D}^{b} \operatorname{Coh}\left(\widetilde{\mathfrak{g}}^{(1)}\right)$ (the supscript ${ }^{(1)}$ denotes the Frobenius twist), extends the action on $\mathcal{D}^{b} \operatorname{Coh}_{\mathcal{B}_{\chi}^{(1)}}\left(\widetilde{\mathfrak{g}}^{(1)}\right)$ considered in BMR2]. Hence, as a consequence of our results in section 4 , the action by intertwining functors on $\mathcal{D}^{b} \operatorname{Mod}_{(\lambda, \chi)}^{\mathrm{fg}}(\mathcal{U} \mathfrak{g})$ of BMR2 factors through an action on $\mathcal{D}^{b} \operatorname{Mod}_{\chi}^{\mathrm{fg}}\left((\mathcal{U} \mathfrak{g})^{\lambda}\right)$ (see 5.1 for notations).

Finally, in section [6 we explain the relation between our results for $\mathbb{k}=\mathbb{C}$ and some classical constructions. In particular, the action on $\mathcal{D}^{b} \operatorname{Coh}(\tilde{\mathcal{N}})$ gives a categorical framework for Ginzburg's isomorphism between the equivariant Ktheory of the Steinberg variety and the extended affine Hecke algebra $\mathcal{H}$, and for Lusztig's construction of irreducible $\mathcal{H}$-modules over $\mathbb{C}$. Also, the action induced on the Grothendieck group of $\mathcal{D}^{b} \operatorname{Coh}_{\mathcal{B}_{\chi}}(\widetilde{\mathcal{N}})$ gives Springer's representations of $W$ on the homology of $\mathcal{B}_{\chi}$.

0.3. To finish this introduction, let us say a few words on the importance of this braid group action. First, its importance was emphasized in Bezrukavnikov's talk at ICM 2006: This action "encodes" the exotic t-structure on $\mathcal{D}^{b} \operatorname{Coh}(\widetilde{\mathfrak{g}})$ and $\mathcal{D}^{b} \operatorname{Coh}(\widetilde{\mathcal{N}})$. In positive characteristic, this t-structure comes from the equivalence with representations of $\mathcal{U} \mathfrak{g}$. It also has an interesting interpretation in characteristic zero (see B2 for details). In fact, our construction will be a step in the proof, by Bezrukavnikov and Mirković, of Lusztig's conjecture relating irreducible $\mathcal{U} \mathfrak{g}$-modules to elements of the canonical basis in the Borel-Moore homology of a Springer fiber ([L3], L4). Similar actions also appear in Gukov and Witten's work on gauge theory and geometric Langlands program (see [GW]), and in Bridgeland's study of stability conditions on triangulated categories (see B2] for details on this point). Finally, we will use this construction in a forthcoming paper to study a certain Koszul duality for modular representations of $\mathfrak{g}$ (see $[\underline{\mathrm{R}}]$ ). For this application, which was our main motivation, we have to assume that the extended Dynkin diagram of each simple factor of $G$ has at least two special points; this excludes factors of type $\mathbf{E}_{8}, \mathbf{F}_{4}$ and $\mathbf{G}_{2}$. Hence, with this application in view, the case of type $\mathbf{G}_{2}$ is not needed.

0.4. Notations. Let $\mathbb{k}$ be an algebraically closed field. Let $R$ be a root system, $W$ its Weyl group, and $G$ the corresponding connected, semi-simple, simply-connected 
algebraic group over $\mathbb{k}$. Let $B$ be a Borel subgroup, $T \subset B$ a maximal torus, $U$ the unipotent radical of $B, B^{+}$the Borel subgroup opposite to $B$, and $U^{+}$its unipotent radical. Let $\mathfrak{g}, \mathfrak{b}, \mathfrak{t}, \mathfrak{n}, \mathfrak{b}^{+}, \mathfrak{n}^{+}$be their respective Lie algebras. Let $R^{+} \subset R$ be the roots in $\mathfrak{n}^{+}$, and $\Phi$ the corresponding set of simple roots. If $\alpha$ is a root, we denote by $U_{\alpha} \subset G$ the corresponding one-parameter subgroup. Let $\mathcal{B}:=G / B$ be the flag variety of $G$, and $\widetilde{\mathcal{N}}:=T^{*} \mathcal{B}$ its cotangent bundle. Geometrically, we have

$$
\tilde{\mathcal{N}}=\left\{(X, g B) \in \mathfrak{g}^{*} \times \mathcal{B} \mid X_{\mid g \cdot \mathfrak{b}}=0\right\} .
$$

We also introduce the "extended" cotangent bundle

$$
\widetilde{\mathfrak{g}}:=\left\{(X, g B) \in \mathfrak{g}^{*} \times \mathcal{B} \mid X_{\mid g \cdot \mathfrak{n}}=0\right\} .
$$

For each positive root $\alpha$, we choose isomorphisms of algebraic groups $u_{\alpha}: \mathbb{k} \stackrel{\sim}{\rightarrow} U_{\alpha}$ and $u_{-\alpha}: \mathbb{k} \stackrel{\sim}{\rightarrow} U_{-\alpha}$ such that for all $t \in T$ we have

$$
t \cdot u_{\alpha}(x) \cdot t^{-1}=u_{\alpha}(\alpha(t) x) \quad \text { and } \quad t \cdot u_{-\alpha}(x) \cdot t^{-1}=u_{-\alpha}\left(\alpha(t)^{-1} x\right),
$$

and such that these morphisms extend to a morphism of algebraic groups $\psi_{\alpha}$ : $\mathrm{SL}(2, \mathbb{k}) \rightarrow G$ such that

$$
\psi_{\alpha}\left(\begin{array}{cc}
1 & x \\
0 & 1
\end{array}\right)=u_{\alpha}(x), \quad \psi_{\alpha}\left(\begin{array}{ll}
1 & 0 \\
x & 1
\end{array}\right)=u_{-\alpha}(x) .
$$

Then we define

$$
n_{\alpha}:=\psi_{\alpha}\left(\begin{array}{cc}
0 & 1 \\
-1 & 0
\end{array}\right) .
$$

This is an element of $N_{G}(T)$ representing the reflection $s_{\alpha} \in W$. We also define $e_{\alpha}:=d\left(u_{\alpha}\right)_{0}(1), e_{-\alpha}:=d\left(u_{-\alpha}\right)_{0}(1)$ and $h_{\alpha}:=\left[e_{\alpha}, e_{-\alpha}\right]$. Let $\mathfrak{s l}(2, \alpha)$ be the image of $\mathfrak{s l}(2, \mathbb{k})$ under $d \psi_{\alpha}$, i.e., the subalgebra with basis $\left\{e_{\alpha}, e_{-\alpha}, h_{\alpha}\right\}$. One has the following well-known formulae for the adjoint action of $G$ on $\mathfrak{g}$, that can be checked in $\mathfrak{s l}(2, \mathbb{k})$ :

$$
\left\{\begin{array}{c}
u_{\alpha}(x) \cdot e_{-\alpha}=e_{-\alpha}+x h_{\alpha}-x^{2} e_{\alpha} \\
n_{\alpha} u_{\alpha}(x) \cdot e_{-\alpha}=x^{2} e_{-\alpha}-x h_{\alpha}-e_{\alpha} \\
u_{\alpha}(x) \cdot h_{\alpha}=h_{\alpha}-2 x e_{\alpha}
\end{array}\right.
$$

Acknowledgements. This work is part of the author's Ph.D. thesis, under the joint supervision of Roman Bezrukavnikov and Patrick Polo. The research on this project was started during a visit to MIT, supported by the École Normale Supérieure de Paris. The author thanks both institutions for their support and hospitality. He deeply thanks Roman Bezrukavnikov for his patience and generosity in explaining his beautiful work and for his useful remarks, and Patrick Polo for his help and encouragement.

\section{Action of the BRAid group by COnVOlution}

1.1. A Bernstein-type presentation of the braid group. Let us introduce some notation concerning Weyl groups and braid groups. We denote by $\mathbb{Y}:=\mathbb{Z} R$ the root lattice of $R$, and by $\mathbb{X}$ its weight lattice. Let $W_{\text {aff }}:=W \ltimes \mathbb{Y}$ be the affine Weyl group, and $W_{\text {aff }}^{\prime}:=W \ltimes \mathbb{X}$ the extended affine Weyl group. We denote by $t_{x} \in W_{\text {aff }}^{\prime}$ the translation corresponding to $x \in \mathbb{X}$. Let $S:=\left\{s_{\alpha}, \alpha \in \Phi\right\}$ be the usual generators of $W$. Let also $S_{\text {aff }} \subset W_{\text {aff }}$ be the usual set of generators of $W_{\text {aff }}$; that is, $S_{\text {aff }}$ contains $S$ together with additional reflections corresponding to the 
highest coroot of each irreducible component of $R$. Then $(W, S)$ and $\left(W_{\text {aff }}, S_{\text {aff }}\right)$ are Coxeter systems. We denote by $\ell$ their length function.

Let $A_{0}=\left\{\lambda \in \mathbb{X} \otimes_{\mathbb{Z}} \mathbb{R} \mid \forall \alpha \in R^{+}, 0<\left\langle\lambda, \alpha^{\vee}\right\rangle<1\right\}$ be the fundamental alcove. If $\Omega \subset W_{\text {aff }}^{\prime}$ is the stabilizer of $A_{0}$ for the standard action on $\mathbb{X} \otimes_{\mathbb{Z}} \mathbb{R}$, we have $W_{\text {aff }}^{\prime} \cong W_{\text {aff }} \rtimes \Omega$. We can use this isomorphism to extend $\ell$ to $W_{\text {aff }}^{\prime}$, setting $\ell(\omega)=0$ for $\omega \in \Omega$. Then, for $w \in W$ and $x \in \mathbb{X}$ ([IM, prop. 1.23]):

$$
\ell\left(w \cdot t_{x}\right)=\sum_{\substack{\alpha \in R^{+} \\ w \alpha \in R^{+}}}\left|\left\langle x, \alpha^{\vee}\right\rangle\right|+\sum_{\substack{\alpha \in R^{+}, w \alpha \in R^{-}}}\left|1+\left\langle x, \alpha^{\vee}\right\rangle\right| .
$$

Now, let us recall the definition of the braid group associated with a Coxeter group $H$, with length $\ell_{H}$. By definition, the braid group $B_{H}$ is the group with generators the $\left\{T_{v}, v \in H\right\}$ and relations $T_{u v}=T_{u} T_{v}$ if $\ell_{H}(u v)=\ell_{H}(u)+\ell_{H}(v)$. In particular, we have the braid group $B_{0}$ associated with $W$, and the affine braid group $B_{\text {aff }}$ associated with $W_{\text {aff }}$. The group $W_{\text {aff }}^{\prime}$ is not a Coxeter group, but we have defined a length function $\ell$ on it. Hence we can use the same recipe to define the extended affine braid group $B_{\text {aff }}^{\prime}$. There are natural inclusions

$$
B_{0} \subset B_{\text {aff }} \subset B_{\text {aff }}^{\prime} \text {. }
$$

Moreover, there is a natural isomorphism $B_{\text {aff }}^{\prime} \cong B_{\text {aff }} \rtimes \Omega$.

There is a canonical section $C: W_{\text {aff }}^{\prime} \rightarrow B_{\text {aff }}^{\prime}$ (which sends $W_{\text {aff into }} B_{\text {aff }}$ and $W$ into $B_{0}$ ) of the canonical morphism $B_{\text {aff }}^{\prime} \rightarrow W_{\text {aff }}^{\prime}$, defined by $C(w):=T_{w}$ (this is not a group morphism). From now on we will no long use the notation $T_{w}$, except when $w=s_{\alpha} \in S$; moreover, in this case, we will simplify $T_{s_{\alpha}}$ in $T_{\alpha}$. We denote by $n_{\alpha, \beta}$ the order of $s_{\alpha} s_{\beta}$ in $W$, for $\alpha, \beta \in \Phi$.

If $\lambda$ and $\mu$ are dominant weights, $\ell\left(t_{\lambda} t_{\mu}\right)=\ell\left(t_{\lambda}\right)+\ell\left(t_{\mu}\right)$; see (1.1.1). Hence

$$
C\left(t_{\lambda} t_{\mu}\right)=C\left(t_{\lambda}\right) C\left(t_{\mu}\right) \text {. }
$$

Let $x \in \mathbb{X}$. We write $x=x_{1}-x_{2}$ with $x_{1}$ and $x_{2}$ dominant weights. Then we set $\theta_{x}:=C\left(t_{x_{1}}\right) C\left(t_{x_{2}}\right)^{-1}$. This does not depend on the chosen decomposition, due to formula (1.1.2). In the Appendix, we prove:

Theorem 1.1.3. $B_{\text {aff }}^{\prime}$ admits a presentation with generators $\left\{T_{\alpha}, \alpha \in \Phi\right\} \cup\left\{\theta_{x}, x \in\right.$ $\mathbb{X}\}$ and relations:

(1) $T_{\alpha} T_{\beta} \cdots=T_{\beta} T_{\alpha} \cdots\left(n_{\alpha, \beta}\right.$ elements on each side);

(2) $\theta_{x} \theta_{y}=\theta_{x+y}$

(3) $T_{\alpha} \theta_{x}=\theta_{x} T_{\alpha}$ if $\left\langle x, \alpha^{\vee}\right\rangle=0$, i.e., $s_{\alpha}(x)=x$;

(4) $\theta_{x}=T_{\alpha} \theta_{x-\alpha} T_{\alpha}$ if $\left\langle x, \alpha^{\vee}\right\rangle=1$, i.e., $s_{\alpha}(x)=x-\alpha$.

This theorem is an analogue of the well-known result of J. Bernstein concerning the corresponding Hecke algebra. We call relations (1) "finite braid relations".

1.2. Convolution. By a variety we mean a reduced, separated scheme of finite type over $\mathbb{k}$ (in particular, we do not assume it is irreducible). If $X$ is a variety, we identify the derived category $\mathcal{D}^{b} \operatorname{Coh}(X)$ with the full subcategory of $\mathcal{D}^{b} \mathrm{QCoh}(X)$ whose objects have coherent cohomology sheaves ([SGA6, II.2.2.2.1]; see also [Bo, VI.2.11] for a sketch of a more elementary proof, following P. Deligne).

If $X$ is a scheme and $i: Z \hookrightarrow X$ a closed subscheme, for simplicity we sometimes write $\mathcal{O}_{Z}$ for $i_{*} \mathcal{O}_{Z}$. We will also sometimes write simply $\left(-\otimes_{X}-\right)$ for $\left(-\otimes_{\mathcal{O}_{X}}-\right)$, and similarly for the derived tensor product. 
Let $X, Y$ be varieties. We denote by $p_{X}: X \times Y \rightarrow X$ and $p_{Y}: X \times Y \rightarrow Y$ the projections. We define the full subcategory

$$
\mathcal{D}_{\text {prop }}^{b} \operatorname{Coh}(X \times Y) \subset \mathcal{D}^{b} \operatorname{Coh}(X \times Y)
$$

as follows: An object of $\mathcal{D}^{b} \operatorname{Coh}(X \times Y)$ belongs to $\mathcal{D}_{\text {prop }}^{b} \operatorname{Coh}(X \times Y)$ if its cohomology sheaves are supported on a closed subscheme $Z \subset X \times Y$ such that the restrictions to $Z$ of $p_{X}$ and $p_{Y}$ are proper. Any $\mathcal{F} \in \mathcal{D}_{\text {prop }}^{b} \operatorname{Coh}(X \times Y)$ gives rise to a functor

$$
F_{X \rightarrow Y}^{\mathcal{F}}:\left\{\begin{array}{ccc}
\mathcal{D}^{b} \operatorname{Coh}(X) & \rightarrow & \mathcal{D}^{b} \operatorname{Coh}(Y) \\
\mathcal{M} & \mapsto & R\left(p_{Y}\right)_{*}\left(\mathcal{F} \otimes^{L}{ }_{X \times Y} p_{X}^{*} \mathcal{M}\right)
\end{array}\right.
$$

(use [H1, II.2.2, II.4.3]). The assignment $\mathcal{F} \mapsto F_{X \rightarrow Y}^{\mathcal{F}}$ is functorial.

Now let $X, Y$ and $Z$ be varieties. We define the convolution product

$$
\text { * : } \mathcal{D}_{\text {prop }}^{b} \operatorname{Coh}(Y \times Z) \times \mathcal{D}_{\text {prop }}^{b} \operatorname{Coh}(X \times Y) \rightarrow \mathcal{D}_{\text {prop }}^{b} \operatorname{Coh}(X \times Z)
$$

by the formula

$$
\mathcal{G} * \mathcal{F}:=R\left(p_{X, Z}\right)_{*}\left(\left(p_{X, Y}\right)^{*} \mathcal{F} \stackrel{L}{\otimes}_{X \times Y \times Z}\left(p_{Y, Z}\right)^{*} \mathcal{G}\right),
$$

where $p_{X, Z}, p_{X, Y}, p_{Y, Z}$ are the natural projections from $X \times Y \times Z$. The following easy result is classical. It can be proved using flat base change ([H1, II.5.12]) and the projection formula ([H1, II.5.6]).

Lemma 1.2.1. Let $\mathcal{F} \in \mathcal{D}_{\text {prop }}^{b} \operatorname{Coh}(X \times Y), \mathcal{G} \in \mathcal{D}_{\text {prop }}^{b} \operatorname{Coh}(Y \times Z)$. Then

$$
F_{Y \rightarrow Z}^{\mathcal{G}} \circ F_{X \rightarrow Y}^{\mathcal{F}} \cong F_{X \rightarrow Z}^{\mathcal{G} * \mathcal{F}} .
$$

In particular, if $X=Y$, the product $*$ endows $\mathcal{D}_{\text {prop }}^{b} \operatorname{Coh}(X \times X)$ with the structure of a monoid, with identity $\Delta_{*} \mathcal{O}_{X}$ (where $\Delta: X \rightarrow X \times X$ is the diagonal embedding). Moreover, $F_{X \rightarrow X}^{(-)}$is a morphism of monoids from this monoid to the monoid of triangulated functors from $\mathcal{D}^{b} \operatorname{Coh}(X)$ to itself.

Assume now that $X$ and $Y$ are non-singular varieties (so that every coherent sheaf has a finite resolution by locally free sheaves of finite type, see for instance [H2, ex. III.6.9]), and let $f: X \rightarrow Y$ be a proper morphism. Let $\Gamma_{f} \subset X \times Y$ be the graph of $f$ (a closed subscheme), and let $\Gamma_{f}^{\prime} \subset Y \times X$ be the image of $\Gamma_{f}$ under the "swap" morphism $X \times Y \rightarrow Y \times X$. Then there exist natural isomorphisms of functors

$$
R f_{*} \cong F_{X \rightarrow Y}^{\mathcal{O}_{\Gamma_{f}}} \text { and } \quad L f^{*} \cong F_{Y \rightarrow X}^{\mathcal{O}_{\Gamma_{f}^{\prime}}}
$$

Hence we have $L f^{*} \circ R f_{*} \cong F_{X \rightarrow X}^{\mathcal{O}_{\Gamma_{f}^{\prime}} * \mathcal{O}_{\Gamma_{f}}}$, with

$$
\mathcal{O}_{\Gamma_{f}^{\prime}} * \mathcal{O}_{\Gamma_{f}} \cong R\left(p_{X, X}\right)_{*}\left(\mathcal{O}_{\Gamma_{f} \times X} \stackrel{L}{\otimes} X \times Y \times X \mathcal{O}_{X \times \Gamma_{f}^{\prime}}\right) .
$$

We also have $\mathrm{Id} \cong F_{X \rightarrow X}^{\Delta * \mathcal{O}_{X}}$.

We denote by $\delta X \subset X \times Y \times X$ the closed subscheme which is the image of $X$ under $x \mapsto(x, f(x), x)$. The following result follows from classical results in the theory of Fourier-Mukai transforms (see [Ca, 5.1], [KT, 4.2]): 
Lemma 1.2.2. The adjunction morphism $L f^{*} \circ R f_{*} \rightarrow \mathrm{Id}$ is induced by the following morphism in $\mathcal{D}_{\text {prop }}^{b} \operatorname{Coh}(X \times X)$ :

$$
\begin{aligned}
R\left(p_{X, X}\right)_{*}\left(\mathcal{O}_{\Gamma_{f} \times X} \stackrel{L}{\otimes}_{X \times Y \times X} \mathcal{O}_{X \times \Gamma_{f}^{\prime}}\right) \rightarrow R\left(p_{X, X}\right)_{*}\left(\mathcal{O}_{\left(\Gamma_{f} \times X\right) \cap\left(X \times \Gamma_{f}^{\prime}\right)}\right) & \\
& \stackrel{\text { res }}{\longrightarrow} R\left(p_{X, X}\right)_{*}\left(\mathcal{O}_{\delta X}\right) \cong \Delta_{*} \mathcal{O}_{X}
\end{aligned}
$$

where the second morphism is induced by restriction, and the first one by the natural

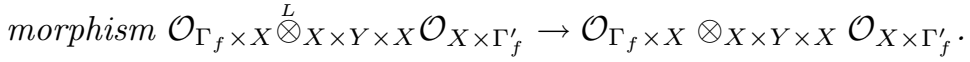

We will also need the following lemma:

Lemma 1.2.3. Let $\mathcal{F} \in \mathcal{D}_{\text {prop }}^{b} \operatorname{Coh}(X \times X)$. Then $\mathcal{O}_{\Gamma_{f}} * \mathcal{F} \cong R(\operatorname{Id} \times f)_{*}(\mathcal{F})$.

Proof. We denote by $p_{i, j}$ the natural projections from $X \times X \times Y$ to $X \times X$ or $X \times Y$, and by $\Delta: X \rightarrow X \times X$ the diagonal embedding. Then we have

$$
\begin{aligned}
& \mathcal{O}_{\Gamma_{f}} * \mathcal{F}=R\left(p_{1,3}\right)_{*}\left(p_{1,2}^{*} \mathcal{F} \stackrel{L}{\otimes}_{X \times X \times Y} p_{2,3}^{*} \mathcal{O}_{\Gamma_{f}}\right), \\
& p_{2,3}^{*} \mathcal{O}_{\Gamma_{f}} \cong R(\operatorname{Id} \times \operatorname{Id} \times f)_{*}(\operatorname{Id} \times \Delta)_{*} \mathcal{O}_{X \times X} .
\end{aligned}
$$

Now, by the projection formula, $\mathcal{O}_{\Gamma_{f}} * \mathcal{F}$ is isomorphic to

$$
R\left(p_{1,3}\right)_{*} R(\operatorname{Id} \times \operatorname{Id} \times f)_{*}(\operatorname{Id} \times \Delta)_{*}\left(L(\operatorname{Id} \times \Delta)^{*} L(\operatorname{Id} \times \operatorname{Id} \times f)^{*}\left(p_{1,2}\right)^{*} \mathcal{F}\right) .
$$

The result follows, since $\left(p_{1,3}\right) \circ(\operatorname{Id} \times \operatorname{Id} \times f) \circ(\operatorname{Id} \times \Delta)=(\operatorname{Id} \times f)$ and $\left(p_{1,2}\right) \circ(\operatorname{Id} \times$ $\operatorname{Id} \times f) \circ(\operatorname{Id} \times \Delta)=\operatorname{Id}_{X \times X}$.

1.3. Action of a group on a category. By an action of a group $A$ on a category $\mathcal{C}$ we mean a weak action, i.e., a group morphism from $A$ to the isomorphism classes of auto-equivalences of the category $\mathcal{C}$ (see BMR2, $[\mathrm{KT}$ ). We will not consider the problem of the compatibility of the isomorphisms of functors corresponding to products of elements of $A$. If $\mathcal{C}=\mathcal{D}^{b} \operatorname{Coh}(X)$ for a variety $X$, to define such an action it is sufficient to construct a morphism of monoids from $A$ to the monoid of isomorphism classes in $\mathcal{D}_{\text {prop }}^{b} \operatorname{Coh}(X \times X)$, endowed with the convolution product $*$.

We will be interested in the case $A=B_{\text {aff }}^{\prime}$ and $X=\widetilde{\mathfrak{g}}$ or $\widetilde{\mathcal{N}}$. Using the presentation of $B_{\text {aff }}^{\prime}$ given in Theorem 1.1.3, to construct the action we only have to define the kernels corresponding to the generators $T_{\alpha}$ and $\theta_{x}$, and to show that they satisfy relations (1) to (4) in $\mathcal{D}_{\text {prop }}^{b} \operatorname{Coh}(X \times X)$, up to isomorphism.

1.4. Construction of kernels. In this paragraph we construct the kernel for the action of $T_{\alpha}$. First, let us introduce some notation. If $X \stackrel{p}{\rightarrow} \mathcal{B}$ is a scheme over $\mathcal{B}$ (resp. if $Y \stackrel{q}{\rightarrow} \mathcal{B} \times \mathcal{B}$ is a scheme over $\mathcal{B} \times \mathcal{B}$ ), and $x, y \in \mathbb{X}$, we denote by $\mathcal{O}_{X}(x)$ (resp. $\left.\mathcal{O}_{Y}(x, y)\right)$ the line bundle $p^{*} \mathcal{O}_{\mathcal{B}}(x)$ (resp. $q^{*}\left(\mathcal{O}_{\mathcal{B}}(x) \otimes \mathcal{O}_{\mathcal{B}}(y)\right)$ ). If $\mathcal{F} \in \mathcal{D}^{b} \operatorname{Coh}(X)\left(\right.$ resp. $\left.\mathcal{D}^{b} \operatorname{Coh}(Y)\right)$, we denote by $\mathcal{F}(x)$ (resp. $\left.\mathcal{F}(x, y)\right)$ the tensor product $\mathcal{F} \otimes_{\mathcal{O}_{X}} \mathcal{O}_{X}(x)$ (resp. $\mathcal{F} \otimes_{\mathcal{O}_{Y}} \mathcal{O}_{Y}(x, y)$ ). We use similar notation for schemes over $\mathcal{B} \times \mathcal{B} \times \mathcal{B}$. If $X \stackrel{a}{\rightarrow} Y$ is a scheme over $Y$, and if $Z \subset Y$ is a locally closed subscheme, we write $\left.X\right|_{Z}$ for the inverse image $a^{-1}(Z)$. Similarly, if $\mathcal{F}$ is a sheaf on $X$, we write $\left.\mathcal{F}\right|_{Z}$ for the restriction of $\mathcal{F}$ to $\left.X\right|_{Z}$.

Let us fix $\alpha \in \Phi$. We denote by $P_{\alpha}$ the standard (i.e. containing $B$ ) parabolic subgroup of $G$ of type $\{\alpha\}$ (see e.g. [Sp, 8.4]), $\mathfrak{p}_{\alpha}$ its Lie algebra, $\mathfrak{p}_{\alpha}^{\mathrm{u}}$ the nilpotent radical of $\mathfrak{p}_{\alpha}$, and $\mathcal{P}_{\alpha}:=G / P_{\alpha}$ the corresponding flag variety. We define

$$
\tilde{\mathfrak{g}}_{\alpha}:=\left\{\left(X, g P_{\alpha}\right) \in \mathfrak{g}^{*} \times \mathcal{P}_{\alpha} \mid X_{\mid g \cdot \mathfrak{p}_{\alpha}^{\mathrm{u}}}=0\right\} .
$$

The projection $\pi_{\alpha}: \mathcal{B} \rightarrow \mathcal{P}_{\alpha}$ induces a morphism $\widetilde{\pi}_{\alpha}: \widetilde{\mathfrak{g}} \rightarrow \widetilde{\mathfrak{g}}_{\alpha}$. 
Let us consider the scheme $\widetilde{\mathfrak{g}} \times_{\widetilde{\mathfrak{g}}_{\alpha}} \widetilde{\mathfrak{g}}$. It is reduced, and it can be described as a variety induced from $B$ to $G$. More precisely, define

$$
\mathscr{R}_{\alpha}:=\left\{(X, g B) \in \mathfrak{g}^{*} \times\left(P_{\alpha} / B\right) \mid X_{\mid \mathfrak{n}+g \cdot \mathfrak{n}}=0\right\} .
$$

We have a natural isomorphism

$$
G \times{ }^{B} \mathscr{R}_{\alpha} \cong \widetilde{\mathfrak{g}} \times_{\widetilde{\mathfrak{g}}_{\alpha}} \widetilde{\mathfrak{g}}
$$

To study the variety $\mathscr{R}_{\alpha}$, we introduce some coordinates. On $\mathfrak{g}^{*}$ we use the coordinates $\left\{e_{\gamma}, \gamma \in R\right\} \cup\left\{h_{\beta}, \beta \in \Phi\right\}$; see 0.4. Consider the open covering $P_{\alpha} / B=\left(U_{\alpha} B / B\right) \cup\left(n_{\alpha} U_{\alpha} B / B\right)$. The morphism $u_{\alpha}$ induces isomorphisms $\mathbb{k} \stackrel{\sim}{\rightarrow}$ $U_{\alpha} \stackrel{\sim}{\rightarrow} U_{\alpha} B / B$ and $\mathbb{k} \stackrel{\sim}{\rightarrow} n_{\alpha} U_{\alpha} \stackrel{\sim}{\rightarrow} n_{\alpha} U_{\alpha} B / B$. We will use the coordinate $t$ on $\mathbb{k}$. Then $\left.\mathscr{R}_{\alpha}\right|_{\left(U_{\alpha} B / B\right)}$ is the set of $(X, t) \in \mathfrak{g}^{*} \times \mathbb{k}$ such that $X$ vanishes on $e_{\gamma}$ for $\gamma \in R^{-}$and on $u_{\alpha}(t) \cdot e_{-\alpha}=e_{-\alpha}+t h_{\alpha}-t^{2} e_{\alpha}$ (see (0.4.1) ). Similarly, $\left.\mathscr{R}_{\alpha}\right|_{\left(n_{\alpha} U_{\alpha} B / B\right)}$ is the set of $(X, t) \in \mathfrak{g}^{*} \times \mathbb{k}$ such that $X$ vanishes on $e_{\gamma}$ for $\gamma \in R^{-}$and on $n_{\alpha} u_{\alpha}(t) \cdot e_{-\alpha}=-e_{\alpha}-t h_{\alpha}+t^{2} e_{-\alpha}$. These are affine varieties, with respective coordinate rings

$$
\begin{aligned}
& \mathbb{k}\left[\left.\mathscr{R}_{\alpha}\right|_{U_{\alpha} B / B}\right] \cong \mathbb{k}\left[h_{\beta}, e_{\gamma}, t, \beta \in \Phi, \gamma \in R^{+}\right] /\left(t\left(h_{\alpha}-t e_{\alpha}\right)\right), \\
& \mathbb{k}\left[\left.\mathscr{R}_{\alpha}\right|_{n_{\alpha} U_{\alpha} B / B}\right] \cong \mathbb{k}\left[h_{\beta}, e_{\gamma}, t, \beta \in \Phi, \gamma \in R^{+}\right] /\left(e_{\alpha}+t h_{\alpha}\right) .
\end{aligned}
$$

In particular, $\mathscr{R}_{\alpha}$ has two irreducible components: one is

$$
\mathscr{D}_{\alpha}:=(\mathfrak{g} / \mathfrak{n})^{*} \times(B / B) \subset \mathfrak{g}^{*} \times\left(P_{\alpha} / B\right),
$$

and the other one is $\mathscr{S}_{\alpha}$, the closure of the complement of $\mathscr{D}_{\alpha}$ in $\mathscr{R}_{\alpha}$. We have the geometric description

$$
\mathscr{S}_{\alpha}=\left\{(X, g B) \in \mathfrak{g}^{*} \times\left(P_{\alpha} / B\right) \mid X_{\mid \mathfrak{n}+g \cdot \mathfrak{n}}=0 \text { and } X\left(h_{\alpha}\right)=0 \text { if } g B=B\right\} .
$$

Hence $\widetilde{\mathfrak{g}} \times_{\tilde{\mathfrak{g}}_{\alpha}} \widetilde{\mathfrak{g}}$ has two irreducible components: $\Delta_{\mathfrak{\mathfrak { g }}}:=G \times{ }^{B} \mathscr{D}_{\alpha}$, which is the diagonal embedding of $\widetilde{\mathfrak{g}}$, and $S_{\alpha}:=G \times{ }^{B} \mathscr{S}_{\alpha}$. Geometrically,

$$
S_{\alpha}=\left\{\begin{array}{l|l}
(X, g B, h B) \in \mathfrak{g}^{*} \times\left(\mathcal{B} \times \mathcal{P}_{\alpha} \mathcal{B}\right) & \begin{array}{l}
X_{\mid g \cdot \mathfrak{n}+h \cdot \mathfrak{n}}=0 \\
\text { and } X\left(g \cdot h_{\alpha}\right)=0 \text { if } g B=h B
\end{array}
\end{array}\right\} .
$$

This second component is a vector bundle over $\mathcal{B} \times_{\mathcal{P}_{\alpha}} \mathcal{B}$, of $\operatorname{rank} \operatorname{dim}(\mathfrak{g} / \mathfrak{n})-1$.

Finally, let us define the closed subscheme $S_{\alpha}^{\prime}$ of $\tilde{\mathcal{N}} \times \widetilde{\mathcal{N}}$ by setting

$$
S_{\alpha}^{\prime}:=S_{\alpha} \cap(\tilde{\mathcal{N}} \times \tilde{\mathcal{N}})
$$

We will see in section 4 that this intersection is a reduced scheme, hence a variety. $S_{\alpha}^{\prime}$ is affine over $\mathcal{B} \times \mathcal{P}_{\alpha} \mathcal{B}$, and it is the induced variety of the subvariety $\mathscr{S}_{\alpha}^{\prime}$ of $\mathfrak{g}^{*} \times\left(P_{\alpha} / B\right)$ defined by

$$
\mathscr{S}_{\alpha}^{\prime}=\left\{(X, g B) \in \mathfrak{g}^{*} \times\left(P_{\alpha} / B\right) \mid X_{\mid \mathfrak{b}+g \cdot \mathfrak{b}}=0\right\} .
$$

The main result of this paper is the following:

Theorem 1.4.1. Assume $G$ has no factor of type $\mathbf{G}_{2}$, and $\operatorname{char}(\mathbb{k}) \neq 2$ if $R$ is not simply-laced. There exists an action of $B_{\text {aff }}^{\prime}$ on $\mathcal{D}^{b} \operatorname{Coh}(\widetilde{\mathfrak{g}})\left(\right.$ resp. $\left.\mathcal{D}^{b} \operatorname{Coh}(\widetilde{\mathcal{N}})\right)$ for which:

(i) The action of $\theta_{x}$ is given by the convolution with kernel $\Delta_{*}\left(\mathcal{O}_{\tilde{\mathfrak{g}}}(x)\right.$ ) (resp. $\left.\Delta_{*}\left(\mathcal{O}_{\widetilde{\mathcal{N}}}(x)\right)\right)$ for $x \in \mathbb{X}$, where $\Delta$ is the diagonal embedding.

(ii) The action of $T_{\alpha}$ is given by the convolution with kernel $\mathcal{O}_{S_{\alpha}}\left(\right.$ resp. $\left.\mathcal{O}_{S_{\alpha}^{\prime}}\right)$ for $\alpha \in \Phi$. 
Moreover, the action of $\left(T_{\alpha}\right)^{-1}$ is the convolution with kernel $\mathcal{O}_{S_{\alpha}}(-\rho, \rho-\alpha)$ (respectively $\mathcal{O}_{S_{\alpha}^{\prime}}(-\rho, \rho-\alpha)$ ).

These actions correspond under the functor $i_{*}: \mathcal{D}^{b} \operatorname{Coh}(\widetilde{\mathcal{N}}) \rightarrow \mathcal{D}^{b} \operatorname{Coh}(\widetilde{\mathfrak{g}})$, where $i: \widetilde{\mathcal{N}} \hookrightarrow \widetilde{\mathfrak{g}}$ is the closed embedding.

The proof of this result occupies most of the rest of the paper. It is clear that the kernels $\Delta_{*}\left(\mathcal{O}_{\widetilde{\mathfrak{g}}}(x)\right)$ (respectively $\Delta_{*}\left(\mathcal{O}_{\widetilde{\mathcal{N}}}(x)\right)$ ) are invertible, and satisfy relation (2) of Theorem 1.1.3 In 1.5 we show that the kernels $\mathcal{O}_{S_{\alpha}}$ for $\alpha \in \Phi$ are also invertible, with inverse $\mathcal{O}_{S_{\alpha}}(-\rho, \rho-\alpha)$. Then, in 1.6 and sections 2 and 3 we show that these kernels satisfy relations (1), (3) and (4) of Theorem 1.1.3, This will prove the assertions concerning the action on $\mathcal{D}^{b} \operatorname{Coh}(\widetilde{\mathfrak{g}})$. In section 4 we explain how one can deduce the assertions concerning the action on $\mathcal{D}^{b} \operatorname{Coh}(\widetilde{\mathcal{N}})$.

1.5. Action of the inverse of the generators. In this paragraph we fix a simple root $\alpha \in \Phi$. The following lemma is very easy, but useful. This result also appears in [L3, 7.19].

Lemma 1.5.1. Let $\lambda \in \mathbb{X}$, such that $\left\langle\lambda, \alpha^{\vee}\right\rangle=0$. The line bundle $\mathcal{O}_{\mathcal{B} \times \mathcal{P}_{\alpha} \mathcal{B}}(\lambda,-\lambda)$ is trivial.

Proof. We have $\mathcal{O}_{\mathcal{B} \times \mathcal{P}_{\alpha} \mathcal{B}}(\lambda,-\lambda) \cong \mathcal{O}_{\mathcal{B} \times \mathcal{P}_{\alpha} \mathcal{B}}(\lambda, 0) \otimes \mathcal{O}_{\mathcal{B} \times \mathcal{P}_{\alpha} \mathcal{B}}(0,-\lambda)$. Moreover, if $p: \mathcal{B} \times_{\mathcal{P}_{\alpha}} \mathcal{B} \rightarrow \mathcal{P}_{\alpha}$ denotes the natural morphism, $\mathcal{O}_{\mathcal{B} \times \mathcal{P}_{\alpha} \mathcal{B}}(\lambda, 0) \cong p^{*} \mathcal{O}_{\mathcal{P}_{\alpha}}(\lambda)$ and $\mathcal{O}_{\mathcal{B} \times \mathcal{P}_{\alpha} \mathcal{B}}(0,-\lambda) \cong p^{*} \mathcal{O}_{\mathcal{P}_{\alpha}}(-\lambda)$. The result follows.

Let us remark in particular that if $\left\langle\lambda, \alpha^{\vee}\right\rangle=\left\langle\mu, \alpha^{\vee}\right\rangle$, then $\mathcal{O}_{\mathcal{B} \times \mathcal{P}_{\alpha} \mathcal{B}}(\lambda, \mu) \cong$ $\mathcal{O}_{\mathcal{B} \times \mathcal{P}_{\alpha} \mathcal{B}}(\mu, \lambda)$. We deduce that $\mathcal{O}_{\mathcal{B} \times \mathcal{P}_{\alpha} \mathcal{B}}(-\rho, \rho-\alpha) \cong \mathcal{O}_{\mathcal{B} \times \mathcal{P}_{\alpha} \mathcal{B}}(\rho-\alpha,-\rho)$, and that $\mathcal{O}_{S_{\alpha}}(-\rho, \rho-\alpha) \cong \mathcal{O}_{S_{\alpha}}(\rho-\alpha,-\rho), \mathcal{O}_{S_{\alpha}^{\prime}}(-\rho, \rho-\alpha) \cong \mathcal{O}_{S_{\alpha}^{\prime}}(\rho-\alpha,-\rho)$.

We will use the following result several times: any finite collection of points of $\mathcal{B}$ is contained in a $B$-translate of $U^{+} B / B$. This follows easily from the fact that if $g_{i} \in G(i=1, \ldots, n)$, then the intersection $\left(\bigcap_{i=1}^{n} g_{i} B U^{+}\right) \cap\left(B U^{+}\right)$is not empty, as an intersection of dense open sets.

Proposition 1.5.2. There exist isomorphisms in $\mathcal{D}_{\text {prop }}^{b} \operatorname{Coh}(\widetilde{\mathfrak{g}} \times \mathfrak{\mathfrak { g }})$ :

$$
\mathcal{O}_{S_{\alpha}} *\left(\mathcal{O}_{S_{\alpha}}(-\rho, \rho-\alpha)\right) \cong \Delta_{*} \mathcal{O}_{\tilde{\mathfrak{g}}} \cong\left(\mathcal{O}_{S_{\alpha}}(-\rho, \rho-\alpha)\right) * \mathcal{O}_{S_{\alpha}} .
$$

Proof. We have $\left(p_{1,2}\right)^{*} \mathcal{O}_{S_{\alpha}} \cong \mathcal{O}_{S_{\alpha} \times \tilde{\mathfrak{g}}}$ and $\left(p_{2,3}\right)^{*} \mathcal{O}_{S_{\alpha}} \cong \mathcal{O}_{\tilde{\mathfrak{g}} \times S_{\alpha}}$. First, let us show that the tensor product

$$
\mathcal{O}_{S_{\alpha} \times \tilde{\mathfrak{g}}} \stackrel{L}{\otimes} \widetilde{\mathfrak{g}}^{3} \mathcal{O}_{\tilde{\mathfrak{g}} \times S_{\alpha}}
$$

is concentrated in degree 0 . As each of these varieties over $\mathcal{B}^{3}$ is the induced variety (from $B$ to $G$ ) of its restriction to $(B / B) \times \mathcal{B}^{2}$, we only have to consider the situation over $(B / B) \times \mathcal{B}^{2}$. By $B$-equivariance, we can even restrict to $(B / B) \times\left(U^{+} B / B\right)^{2} \cong$ $\left(U^{+}\right)^{2}$ (see the remark above).

Let us choose some coordinates on $\left.\widetilde{\mathfrak{g}}^{3}\right|_{(B / B) \times\left(U^{+} B / B\right)^{2}}$. We have isomorphisms $\left.\widetilde{\mathfrak{g}}\right|_{U^{+B / B}} \stackrel{\sim}{\longrightarrow}\left(\mathfrak{b}^{+}\right)^{*} \times\left(U^{+} B / B\right)$ (induced by restriction), and $\left.\widetilde{\mathfrak{g}}\right|_{B / B} \cong\left(\mathfrak{b}^{+}\right)^{*}$. Hence on the fibers, isomorphic to $\left(\left(\mathfrak{b}^{+}\right)^{*}\right)^{3}$, we choose coordinates $e_{\gamma}^{(j)}, h_{\beta}^{(j)}\left(\gamma \in R^{+}\right.$, $\beta \in \Phi, j \in\{1,2,3\})$ which are copies of the elements of the basis of $\mathfrak{g}$ defined in 0.4. The multiplication induces an isomorphism $U_{(\alpha)}^{+} \times U_{\alpha} \stackrel{\sim}{\rightarrow} U^{+}$, where $U_{(\alpha)}^{+}$is the product of the $U_{\gamma}$ for $\gamma \in R^{+}-\{\alpha\}$ (this is the unipotent radical of the parabolic subgroup opposite to $P_{\alpha}$ ). Hence, $u_{\alpha}$ and multiplication induce an isomorphism 
$U_{(\alpha)}^{+} \times \mathbb{k} \stackrel{\sim}{\rightarrow} U^{+}$. Using this, we choose coordinates $\left(u^{(j)}, t^{(j)}\right)$ on $U^{+}$, considered as the base of the $j$-th copy of $\widetilde{\mathfrak{g}}(j=2,3)$.

Then $\left.\left(S_{\alpha} \times \widetilde{\mathfrak{g}}\right)\right|_{(B / B) \times(U+B / B)^{2}}$ is defined in $\left.(\widetilde{\mathfrak{g}})^{3}\right|_{(B / B) \times\left(U^{+} B / B\right)^{2}}$ by the equations $u^{(2)}=1, h_{\beta}^{(1)}=h_{\beta}^{(2)}(\beta \in \Phi), e_{\gamma}^{(1)}=e_{\gamma}^{(2)}\left(\gamma \in R^{+}\right)$and $h_{\alpha}^{(1)}-t^{(2)} e_{\alpha}^{(1)}=0$ (see 1.4). It is clear that these equations form a regular sequence in $\mathbb{k}\left[\left.\tilde{\mathfrak{g}}^{3}\right|_{(B / B) \times\left(U^{+} B / B\right)^{2}}\right]$. Similarly, $\left.\left(\tilde{\mathfrak{g}} \times S_{\alpha}\right)\right|_{(B / B) \times\left(U^{+} B / B\right)^{2}}$ is defined by the equations $u^{(2)}=u^{(3)}, h_{\beta}^{(2)}=$ $h_{\beta}^{(3)}(\beta \in \Phi), e_{\gamma}^{(2)}=e_{\gamma}^{(3)}\left(\gamma \in R^{+}\right)$and $u^{(3)} \cdot\left(h_{\alpha}^{(3)}-\left(t^{(2)}+t^{(3)}\right) e_{\alpha}^{(3)}\right)=0$. Now the union of these two sequences is again a regular sequence, and defines a reduced scheme. Hence the derived tensor product (1.5.3) is concentrated in degree 0, and equals the sheaf of functions on the subvariety $V_{\alpha}:=\left(S_{\alpha} \times \widetilde{\mathfrak{g}}\right) \cap\left(\widetilde{\mathfrak{g}} \times S_{\alpha}\right)$ of $\widetilde{\mathfrak{g}}^{3}$. Now we compute

$$
R\left(p_{1,3}\right)_{*}\left(\mathcal{O}_{V_{\alpha}}(\rho-\alpha,-\rho, 0)\right) \text { and } R\left(p_{1,3}\right)_{*}\left(\mathcal{O}_{V_{\alpha}}(0,-\rho, \rho-\alpha)\right) .
$$

The following result will be proved later:

Lemma 1.5.5. The variety $V_{\alpha}$ has two irreducible components: $V_{\alpha}^{1}$, which is the restriction of $V_{\alpha}$ to the partial diagonal $\Delta_{\mathcal{B}}^{1,3} \subset \mathcal{B}^{3}$, and $V_{\alpha}^{2}$, which has the following geometric description:

$$
V_{\alpha}^{2}=\left\{\left(X, g_{1} B, g_{2} B, g_{3} B\right) \in \mathfrak{g}^{*} \times\left(\mathcal{B} \times \mathcal{P}_{\alpha} \mathcal{B} \times \mathcal{P}_{\alpha} \mathcal{B}\right) \mid X_{\mid g_{1} \cdot(\mathfrak{n}+\mathfrak{s l}(2, \alpha))}=0\right\} .
$$

Moreover, there exist exact sequences of sheaves

$$
\begin{aligned}
& \mathcal{O}_{V_{\alpha}^{1}} \hookrightarrow \mathcal{O}_{V_{\alpha}}(\rho-\alpha,-\rho, 0) \rightarrow \mathcal{O}_{V_{\alpha}^{2}}(\rho-\alpha,-\rho, 0), \\
& \mathcal{O}_{V_{\alpha}^{1}} \hookrightarrow \mathcal{O}_{V_{\alpha}}(0,-\rho, \rho-\alpha) \rightarrow \mathcal{O}_{V_{\alpha}^{2}}(0,-\rho, \rho-\alpha) .
\end{aligned}
$$

It follows that to compute the direct images (1.5.4) we only have to compute $R\left(p_{1,3}\right)_{*}\left(\mathcal{O}_{V_{\alpha}^{1}}\right), R\left(p_{1,3}\right)_{*}\left(\mathcal{O}_{V_{\alpha}^{2}}(\rho-\alpha,-\rho, 0)\right)$ and $R\left(p_{1,3}\right)_{*}\left(\mathcal{O}_{V_{\alpha}^{2}}(0,-\rho, \rho-\alpha)\right)$. But $R\left(p_{1,3}\right)_{*}\left(\mathcal{O}_{V_{\alpha}^{2}}(\rho-\alpha,-\rho, 0)\right)=R\left(p_{1,3}\right)_{*}\left(\mathcal{O}_{V_{\alpha}^{2}}(0,-\rho, \rho-\alpha)\right) \stackrel{\alpha}{=} 0$ because $p_{1,3}$ is a locally trivial fibration of fiber $\mathbb{P}_{\mathbb{k}}^{1}$ on $V_{\alpha}^{2}$, and the sheaf on this fiber is $\mathcal{O}_{\mathbb{P}^{1}}(-1)$. To conclude, we only have to show that $R\left(p_{1,3}\right)_{*}\left(\mathcal{O}_{V_{\alpha}^{1}}\right) \cong \Delta_{*} \mathcal{O}_{\widetilde{\mathfrak{g}}}$.

By local triviality we only have to consider the morphism

$$
q_{1,3}:\left.V_{\alpha}\right|_{(B / B) \times\left(P_{\alpha} / B\right) \times(B / B)} \rightarrow(\mathfrak{g} / \mathfrak{n})^{*} .
$$

Then define $M:=\left(\mathfrak{s l}(2, \alpha) /\left(\mathbb{k} e_{-\alpha}\right)\right)^{*}$, and choose a vector subspace $M^{\prime} \subset \mathfrak{g} / \mathfrak{n}$ such that $\mathfrak{g} / \mathfrak{n} \cong M^{*} \oplus M^{\prime}$. Let $E=\{(D, x) \in \mathbb{P}(M) \times M \mid x \in D\}$ be the tautological line bundle on $\mathbb{P}(M)$. Then the morphism $q_{1,3}$ identifies with the product of $\operatorname{Id}_{\left(M^{\prime}\right)^{*}}$ and the canonical projection $f: E \rightarrow M$. Hence we only have to show that $R f_{*} \mathcal{O}_{E} \cong \mathcal{O}_{M}$. As $M$ is affine we only have to consider the global sections; but the direct image of $\mathcal{O}_{E}$ under the canonical projection to $\mathbb{P}(M)$ is $\bigoplus_{m \geq 0} \mathcal{O}_{\mathbb{P}(M)}(m)$, whose global sections are $S\left(M^{*}\right)$.

This completes the proof of Proposition 1.5.2, assuming Lemma 1.5.5.

Proof of Lemma 1.5.5. Consider the subvariety $\mathscr{V}_{\alpha}$ of $\mathfrak{g}^{*} \times\left(P_{\alpha} / B\right) \times\left(P_{\alpha} / B\right)$ :

$$
\begin{aligned}
\mathscr{V}_{\alpha}:=\left\{(X, g B, h B) \in \mathfrak{g}^{*} \times\left(P_{\alpha} / B\right) \times\left(P_{\alpha} / B\right) \mid X_{\mid \mathfrak{n}+g \cdot \mathfrak{n}+h \cdot \mathfrak{n}}\right. & =0, \\
X\left(h_{\alpha}\right)=0 & \text { if } \left.g B=B \text { and } X\left(g \cdot h_{\alpha}\right)=0 \text { if } g B=h B\right\} .
\end{aligned}
$$

We have an isomorphism $V_{\alpha} \cong G \times{ }^{B} \mathscr{V}_{\alpha}$. On $\left(P_{\alpha} / B\right)^{2}$ we use the open covering $\left(P_{\alpha} / B\right)^{2}=\left(U_{\alpha} B / B\right)^{2} \cup\left(n_{\alpha} U_{\alpha} B / B\right)^{2} \cup\left[\left(U_{\alpha} B / B\right) \times\left(n_{\alpha} U_{\alpha} B / B\right)\right] \cup\left[\left(n_{\alpha} U_{\alpha} B / B\right) \times\right.$ $\left(U_{\alpha} B / B\right)$. Each of these open sets is isomorphic to $\mathbb{k}^{2}$, via $u_{\alpha}$. We use the 
coordinates $t^{(1)}$ and $t^{(2)}$ on $\left(P_{\alpha} / B\right)^{2}$, and $\left\{e_{\gamma}, \gamma \in R, h_{\beta}, \beta \in \Phi\right\}$ on $\mathfrak{g}^{*}$. The change of coordinates on the intersection $\left(U_{\alpha} B / B\right) \cap\left(n_{\alpha} U_{\alpha} B / B\right)$ is given by $t \mapsto-\frac{1}{t}$ (this can be checked in $\mathrm{SL}(2, \mathbb{k}))$.

The restriction $\left.\mathscr{V}_{\alpha}\right|_{\left(U_{\alpha} B / B\right)^{2}}$ is defined in $\mathfrak{g}^{*} \times \mathbb{k}^{2}$ by the equations $e_{\gamma}=0(\gamma \in$ $R^{-}$), $h_{\alpha}-t^{(1)} e_{\alpha}=0$ and $h_{\alpha}-\left(t^{(1)}+t^{(2)}\right) e_{\alpha}=0$ (see the preceding proof). This last equation can be replaced by $t^{(2)} e_{\alpha}=0$. Similarly, $\left.\mathscr{V}_{\alpha}\right|_{\left(n_{\alpha} U_{\alpha} B / B\right)^{2}}$ is defined in $\mathfrak{g}^{*} \times \mathbb{k}^{2}$ by the equations $e_{\gamma}=0\left(\gamma \in R^{-}\right), e_{\alpha}+t^{(1)} h_{\alpha}=0$ and $h_{\alpha}=0$. Over $\left(U_{\alpha} B / B\right) \times\left(n_{\alpha} U_{\alpha} B / B\right)$, the equations are $e_{\gamma}=0\left(\gamma \in R^{-}\right), h_{\alpha}-t^{(1)} e_{\alpha}=0$ and $e_{\alpha}=0$. Finally, $\left.\mathscr{V}_{\alpha}\right|_{\left(n_{\alpha} U_{\alpha} B / B\right) \times\left(U_{\alpha} B / B\right)}$ is defined by $e_{\gamma}=0\left(\gamma \in R^{-}\right)$, $e_{\alpha}+t^{(1)} h_{\alpha}=0$ and $t^{(2)} h_{\alpha}=0$. These equations show that $\mathscr{V}_{\alpha}$ has two irreducible components: $\mathscr{V}_{\alpha}^{1}$, which is the restriction of $\mathscr{V}_{\alpha}$ to $\left(P_{\alpha} / B\right) \times(B / B) \subset\left(P_{\alpha} / B\right)^{2}$, and $\mathscr{V}_{\alpha}^{2}$, which has the following geometric description:

$$
\mathscr{V}_{\alpha}^{2}=\left\{(X, g B, h B) \in \mathfrak{g}^{*} \times\left(P_{\alpha} / B\right)^{2} \mid X_{\mid \mathfrak{n}+\mathfrak{s l}(2, \alpha)}=0\right\} .
$$

The varieties $\mathscr{V}_{\alpha}, \mathscr{V}_{\alpha}^{1}$ and $\mathscr{V}_{\alpha}^{2}$ are affine over $\left(U_{\alpha} B / B\right)^{2}$, with respective rings of functions $\mathbb{k}\left[e_{\gamma}, h_{\beta}, t^{(i)}\right] /\left(h_{\alpha}-t^{(1)} e_{\alpha}, t^{(2)} e_{\alpha}\right), \mathbb{k}\left[e_{\gamma}, h_{\beta}, t^{(i)}\right] /\left(h_{\alpha}-t^{(1)} e_{\alpha}, t^{(2)}\right)$ and $\mathbb{k}\left[e_{\gamma}, h_{\beta}, t^{(i)}\right] /\left(h_{\alpha}-t^{(1)} e_{\alpha}, e_{\alpha}\right)$. Hence the multiplication by $e_{\alpha}$ and the natural quotient induce an exact sequence of sheaves

$$
\left.\left.\left.\mathcal{O}_{\mathscr{V}_{\alpha}^{1}}\right|_{\left(U_{\alpha} B / B\right)^{2}} \hookrightarrow \mathcal{O}_{\mathscr{V}_{\alpha}}\right|_{\left(U_{\alpha} B / B\right)^{2}} \rightarrow \mathcal{O}_{\mathscr{V}_{\alpha}^{2}}\right|_{\left(U_{\alpha} B / B\right)^{2}}
$$

Multiplication by $h_{\alpha}$ induces a similar sequence on $\left(n_{\alpha} U_{\alpha} B / B\right) \times\left(U_{\alpha} B / B\right)$.

The element $e_{\alpha} \in \mathbb{k}\left[\left.\mathscr{V}_{\alpha}\right|_{\left(U_{\alpha} B / B\right)^{2}}\right]$ is sent to 0 when restricted to the open sets $\left(n_{\alpha} U_{\alpha} B / B\right)^{2}$ or $\left(U_{\alpha} B / B\right) \times\left(n_{\alpha} U_{\alpha} B / B\right)$, and to $-t^{(1)} h_{\alpha}$ when restricted to $\left(n_{\alpha} U_{\alpha} B / B\right) \times\left(U_{\alpha} B / B\right)$. Hence the preceding exact sequences glue to give an exact sequence of (non- $B$-equivariant) sheaves

$$
\mathcal{O}_{\mathscr{V}_{\alpha}^{1}} \otimes_{\mathcal{O}_{\left(P_{\alpha} / B\right)^{2}}} \mathcal{O}_{\left(P_{\alpha} / B\right)^{2}}(1,0) \hookrightarrow \mathcal{O}_{\mathscr{V}_{\alpha}} \rightarrow \mathcal{O}_{\mathscr{V}_{\alpha}^{2}}
$$

where we have used the isomorphism $P_{\alpha} / B \cong \mathbb{P}_{\mathbb{k}}^{1}$. Now consider the $B$-equivariant structures. The second morphism in this sequence is obviously equivariant. We have $\mathcal{O}_{\left(P_{\alpha} / B\right)^{2}}(1,0)=\mathcal{O}_{\left(P_{\alpha} / B\right)^{2}}(\rho, 0)$, and the first arrow of the exact sequence comes by definition from a $B$-equivariant morphism $\mathbb{k}_{B}(\alpha-\rho) \otimes_{\mathbb{k}} \mathcal{O}_{\mathscr{V}_{\alpha}^{1}}(\rho, 0) \hookrightarrow \mathcal{O}_{\mathscr{V}_{\alpha}}$. Hence we obtain the exact sequence of $B$-equivariant sheaves

$$
\mathbb{k}_{B}(\alpha-\rho) \otimes_{\mathbb{k}} \mathcal{O}_{\mathscr{V}_{\alpha}^{1}}(\rho, 0) \hookrightarrow \mathcal{O}_{\mathscr{V}_{\alpha}} \rightarrow \mathcal{O}_{\mathscr{V}_{\alpha}^{2}} .
$$

Inducing from $B$ to $G$, this gives the first exact sequence of the lemma. To prove the second one, we observe that we also have an exact sequence

$$
\mathcal{O}_{V_{\alpha}^{1}}(\alpha-\rho, 0, \rho-\alpha) \hookrightarrow \mathcal{O}_{V_{\alpha}}(0,-\rho, \rho-\alpha) \rightarrow \mathcal{O}_{V_{\alpha}^{2}}(0,-\rho, \rho-\alpha) .
$$

As $V_{\alpha}^{1}$ is supported on $\Delta_{\mathcal{B}}^{1,3} \subset \mathcal{B}^{3}$, the first sheaf equals $\mathcal{O}_{V_{\alpha}^{1}}$.

Remark 1.5.6. In these two results, one can replace $\rho$ by any $\lambda \in \mathbb{X}$ such that $\left\langle\lambda, \alpha^{\vee}\right\rangle=1$. This follows either from the proofs, or from Lemma 1.5.1.

1.6. First relations. In this paragraph we show that the kernels of Theorem 1.4.1 for the action on $\mathcal{D}^{b} \operatorname{Coh}(\widetilde{\mathfrak{g}})$ satisfy relations (3) and (4) of the presentation of $B_{\text {aff }}^{\prime}$ given by Theorem 1.1.3.

Let us consider relation (3). Let $\alpha \in \Phi$ and $x \in \mathbb{X}$ be such that $\left\langle x, \alpha^{\vee}\right\rangle=0$. We have to show that $\mathcal{O}_{S_{\alpha}}$ commutes with $\Delta_{*} \mathcal{O}_{\mathfrak{\mathfrak { g }}}(x)$. But

$$
\mathcal{O}_{S_{\alpha}} *\left(\Delta_{*} \mathcal{O}_{\widetilde{\mathfrak{g}}}(x)\right) \cong \mathcal{O}_{S_{\alpha}}(x, 0), \quad\left(\Delta_{*} \mathcal{O}_{\mathfrak{\mathfrak { g }}}(x)\right) * \mathcal{O}_{S_{\alpha}} \cong \mathcal{O}_{S_{\alpha}}(0, x),
$$


and $\mathcal{O}_{\mathcal{B} \times \mathcal{P}_{\alpha} \mathcal{B}}(x, 0)=\mathcal{O}_{\mathcal{B} \times \mathcal{P}_{\alpha} \mathcal{B}}(0, x)$ by Lemma 1.5.1. Taking the inverse image to $S_{\alpha}$, we obtain the result.

Now, consider relation (4). Let $\alpha \in \Phi$ and $x \in \mathbb{X}$ be such that $\left\langle x, \alpha^{\vee}\right\rangle=1$. We have to prove that $\Delta_{*} \mathcal{O}_{\mathfrak{\mathfrak { g }}}(x) \cong \mathcal{O}_{S_{\alpha}} *\left(\Delta_{*} \mathcal{O}_{\widetilde{\mathfrak{g}}}(x-\alpha)\right) * \mathcal{O}_{S_{\alpha}}$. Due to Proposition 1.5.2, this is equivalent to proving

$$
\left(\Delta_{*} \mathcal{O}_{\tilde{\mathfrak{g}}}(x)\right) *\left(\mathcal{O}_{S_{\alpha}}(-\rho, \rho-\alpha)\right) \cong\left(\mathcal{O}_{S_{\alpha}}\right) *\left(\Delta_{*} \mathcal{O}_{\widetilde{\mathfrak{g}}}(x-\alpha)\right) .
$$

We have $\mathcal{O}_{\mathcal{B} \times \mathcal{P}_{\alpha} \mathcal{B}}(-\rho, x+\rho-\alpha) \cong \mathcal{O}_{\mathcal{B} \times \mathcal{P}_{\alpha} \mathcal{B}}(x-\alpha, 0)$ by Lemma 1.5.1 again. The result follows, since

$$
\begin{aligned}
\left(\Delta_{*} \mathcal{O}_{\tilde{\mathfrak{g}}}(x)\right) *\left(\mathcal{O}_{S_{\alpha}}(-\rho, \rho-\alpha)\right) & \cong \mathcal{O}_{S_{\alpha}}(-\rho, x+\rho-\alpha), \\
\left(\mathcal{O}_{S_{\alpha}}\right) *\left(\Delta_{*} \mathcal{O}_{\tilde{\mathfrak{g}}}(x-\alpha)\right) & \cong \mathcal{O}_{S_{\alpha}}(x-\alpha, 0) .
\end{aligned}
$$

1.7. More notation. In this paragraph we introduce notations concerning Schubert varieties and Demazure resolutions (following $[\mathrm{BK}]$ ).

If $w \in W$, we denote by $X_{w}$ the corresponding Schubert variety. This is the closure of $B w B / B$ in $\mathcal{B}$. Similarly, we denote by $\mathcal{X}_{w}$ the closure of the $G$-orbit of $(B / B, w B / B)$ in $\mathcal{B} \times \mathcal{B}$, called $G$-Schubert variety. Its points are the couples of Borel subgroups of $G$ in relative position at most $w$ in the Bruhat order. It identifies with $G \times \times^{B} X_{w}$ under the isomorphism $G \times{ }^{B} \mathcal{B} \cong \mathcal{B} \times \mathcal{B}$.

For $w=s_{1} \cdots s_{n}$ a reduced expression in $W$, let $Z_{\left(s_{1}, \cdots, s_{n}\right)}$ be the associated Demazure resolution of the Schubert variety $X_{w}$ (as defined in $[\mathrm{BK}]$ ). Also let $\mathcal{Z}_{\left(s_{1}, \cdots, s_{n}\right)}$ be the induction from $B$ to $G$ of this resolution, which is a resolution of $\mathcal{X}_{w}$, and let $\Phi_{\left(s_{1}, \cdots, s_{n}\right)}: \mathcal{Z}_{\left(s_{1}, \cdots, s_{n}\right)} \rightarrow \mathcal{X}_{w}$ be the associated morphism. If $s_{j}$ is the reflection associated with the simple root $\alpha_{j} \in \Phi$ for any $j=1, \ldots, n$, and $\mathcal{P}_{j}:=G / P_{j}$ for $P_{j}$ the standard parabolic subgroup of $G$ of type $\left\{\alpha_{j}\right\}$, then we have an isomorphism $\mathcal{Z}_{\left(s_{1}, \ldots, s_{n}\right)} \cong \mathcal{B} \times \mathcal{P}_{1} \mathcal{B} \times \mathcal{P}_{2} \cdots \times \times_{\mathcal{P}_{n}} \mathcal{B}$, and $\Phi_{\left(s_{1}, \ldots, s_{n}\right)}$ identifies with the restriction of the projection $p_{1, n+1}: \mathcal{B}^{n+1} \rightarrow \mathcal{B}^{2}$. Let $\widetilde{\mathcal{Z}}_{\left(s_{1}, \cdots, s_{n}\right)}$ be the intersection

$$
\left(S_{\alpha_{1}} \times \widetilde{\mathfrak{g}}^{n-1}\right) \cap\left(\widetilde{\mathfrak{g}} \times S_{\alpha_{2}} \times \widetilde{\mathfrak{g}}^{n-2}\right) \cap \cdots \cap\left(\widetilde{\mathfrak{g}}^{n-1} \times S_{\alpha_{n}}\right),
$$

a subscheme of $\widetilde{\mathfrak{g}}^{n+1}$.

In the next two sections we prove the finite braid relations, first in the case when the simple roots $\alpha$ and $\beta$ generate a root system of type $\mathbf{A}_{2}$, and then in the case when they generate a system of type $\mathbf{B}_{2}$. The much easier case of a root system of type $\mathbf{A}_{1} \times \mathbf{A}_{1}$ is left to the reader.

\section{Finite braid Relations for type $\mathbf{A}_{2}$}

Let $\alpha$ and $\beta$ be simple roots generating a root system of type $\mathbf{A}_{2}$, i.e., such that $\left\langle\alpha, \beta^{\vee}\right\rangle=\left\langle\beta, \alpha^{\vee}\right\rangle=-1$. It is well known (see e.g. [Sp, 8.2.3]) that there exists $c \in \mathbb{k}^{\times}$such that

$$
\forall x, y \in \mathbb{k}, \quad\left(u_{\alpha}(x), u_{\beta}(y)\right)=u_{\alpha+\beta}(c x y) .
$$

The following formulae for the adjoint action of $G$ on $\mathfrak{g}$ follow easily:

$$
\begin{array}{ll}
u_{\alpha}(x) \cdot e_{\beta}=e_{\beta}+c x e_{\alpha+\beta}, & u_{\alpha+\beta}(x) \cdot h_{\beta}=h_{\beta}-x e_{\alpha+\beta}, \\
u_{\alpha}(x) \cdot h_{\beta}=h_{\beta}+x e_{\alpha}, & u_{\alpha+\beta}(x) \cdot e_{-\beta}=e_{-\beta}+(x / c) e_{\alpha} .
\end{array}
$$

We also have $\left[e_{\alpha}, e_{\beta}\right]=c e_{\alpha+\beta}$. The corresponding formulae with $\alpha$ and $\beta$ interchanged are obtained by replacing $c$ by $-c$. Finally, note that $h_{\alpha+\beta}=h_{s_{\alpha}(\beta)}=$ $s_{\alpha}\left(h_{\beta}\right)=h_{\alpha}+h_{\beta}$. 
In this section we prove that

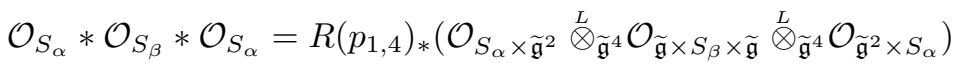

is invariant under the exchange of $\alpha$ and $\beta$ (where $p_{1,4}: \widetilde{\mathfrak{g}}^{4} \rightarrow \widetilde{\mathfrak{g}}^{2}$ is the natural projection). In fact, we calculate this complex of sheaves explicitly.

\subsection{Derived tensor product.}

Lemma 2.1.1. There exist isomorphisms

$$
\begin{aligned}
& \mathcal{O}_{S_{\alpha} \times \widetilde{\mathfrak{g}}^{2}} \stackrel{L}{\otimes}_{\widetilde{\mathfrak{g}}^{4}} \mathcal{O}_{\widetilde{\mathfrak{g}} \times S_{\beta} \times \widetilde{\mathfrak{g}}} \stackrel{L}{\otimes}_{\widetilde{\mathfrak{g}}^{4}} \mathcal{O}_{\widetilde{\mathfrak{g}}^{2} \times S_{\alpha}} \cong \mathcal{O}_{\widetilde{\mathcal{Z}}_{\left(s_{\alpha}, s_{\beta}, s_{\alpha}\right)}}, \\
& \mathcal{O}_{S_{\beta} \times \widetilde{\mathfrak{g}}^{2}} \stackrel{L}{\otimes}_{\mathfrak{\mathfrak { g }}^{4}} \mathcal{O}_{\widetilde{\mathfrak{g}} \times S_{\alpha} \times \widetilde{\mathfrak{g}}} \stackrel{L}{\otimes}_{\widetilde{\mathfrak{g}}^{4}} \mathcal{O}_{\widetilde{\mathfrak{g}}^{2} \times S_{\beta}} \cong \mathcal{O}_{\widetilde{\mathcal{Z}}_{\left(s_{\beta}, s_{\alpha}, s_{\beta}\right)}} .
\end{aligned}
$$

Moreover, the schemes $\widetilde{\mathcal{Z}}_{\left(s_{\alpha}, s_{\beta}, s_{\alpha}\right)}$ and $\widetilde{\mathcal{Z}}_{\left(s_{\beta}, s_{\alpha}, s_{\beta}\right)}$ are integral, i.e., reduced and irreducible.

Proof. We write the proof in the first case only, the second one being similar (replace $c$ by $-c$ ). As in the proof of Proposition 1.5.2, we only have to study the situation over $(B / B) \times\left(U^{+} B / B\right)^{3}$. Let us choose an order on $R^{+}$such that the last three roots are $\alpha+\beta, \beta, \alpha$ (in this order). Let $P_{\alpha}, P_{\beta}, P_{\alpha, \beta}$ be the standard parabolic subgroups of $G$ associated to $\{\alpha\},\{\beta\}$ and $\{\alpha, \beta\}$. We denote by $U_{(\alpha)}^{+}, U_{(\beta)}^{+}, U_{(\alpha, \beta)}^{+}$ the product of the $U_{\gamma}$ for $\gamma \in R^{+}-\{\alpha\}, \gamma \in R^{+}-\{\beta\}, \gamma \in R^{+}-\{\alpha, \beta, \alpha+\beta\}$ (these are the unipotent radicals of the parabolic subgroups opposite to $P_{\alpha}, P_{\beta}, P_{\alpha, \beta}$ ). We have an isomorphism $U^{+} \cong \prod_{\gamma \in R^{+}} U_{\gamma}$. Via this isomorphism, the restriction to $U^{+} B / B$ of the projections $G / B \rightarrow G / P_{\alpha}$ and $G / B \rightarrow G / P_{\alpha, \beta}$ become the natural projections $U_{(\alpha)}^{+} \times U_{\alpha} \rightarrow U_{(\alpha)}^{+}$and $U_{(\alpha, \beta)}^{+} \times U_{\alpha+\beta} \times U_{\beta} \times U_{\alpha} \rightarrow U_{(\alpha, \beta)}^{+}$. The restriction of the projection $G / B \rightarrow G / P_{\beta}$ becomes

$$
\left\{\begin{aligned}
U_{(\alpha, \beta)}^{+} \times U_{\alpha+\beta} \times U_{\beta} \times U_{\alpha} & \rightarrow U_{(\beta)}^{+} \cong U_{(\alpha, \beta)}^{+} \times U_{\alpha+\beta} \times U_{\alpha} \\
\left(u, u_{\alpha+\beta}(x), u_{\beta}(y), u_{\alpha}(z)\right) & \mapsto\left(u, u_{\alpha+\beta}(x-c y z), u_{\alpha}(z)\right) .
\end{aligned}\right.
$$

As in Proposition 1.5.2, as coordinates on $\left.\widetilde{\mathfrak{g}}^{4}\right|_{(B / B) \times\left(U^{+} B / B\right)^{3}}$ we use $u^{(j)} \in U_{(\alpha, \beta)}^{+}$, $x^{(j)}, y^{(j)}, z^{(j)} \in \mathbb{k}$ on the base, and $h_{\delta}^{(j)}(\delta \in \Phi)$ and $e_{\gamma}^{(j)}\left(\gamma \in R^{+}\right)$on the fiber of the $j$-th copy of $\widetilde{\mathfrak{g}}$ (we do not use the coordinates $u^{(1)}, x^{(1)}, y^{(1)}$ and $z^{(1)}$ because in the first copy of $\widetilde{\mathfrak{g}}$ we only consider the fiber over $B / B$ ).

In these coordinates, $\left.\left.\left(S_{\alpha} \times \widetilde{\mathfrak{g}}^{2}\right)\right|_{(B / B) \times\left(U^{+} B / B\right)^{3}} \subset\left(\widetilde{\mathfrak{g}}^{4}\right)\right|_{(B / B) \times\left(U^{+} B / B\right)^{3}}$ is defined by the equations

$$
u^{(2)}=1, x^{(2)}=0, y^{(2)}=0, h_{\delta}^{(1)}=h_{\delta}^{(2)}, e_{\gamma}^{(1)}=e_{\gamma}^{(2)}\left(\delta \in \Phi, \gamma \in R^{+}\right)
$$

and

$$
h_{\alpha}^{(1)}-z^{(2)} e_{\alpha}^{(1)}=0
$$

Similarly, $\left.\left.\left(\widetilde{\mathfrak{g}} \times S_{\beta} \times \widetilde{\mathfrak{g}}\right)\right|_{(B / B) \times(U+B / B)^{3}} \subset\left(\widetilde{\mathfrak{g}}^{4}\right)\right|_{(B / B) \times(U+B / B)^{3}}$ is defined by the equations

$$
\left\{\begin{array}{l}
u^{(3)}=u^{(2)}, x^{(2)}-c y^{(2)} z^{(2)}=x^{(3)}-c y^{(3)} z^{(3)} \\
z^{(2)}=z^{(3)}, h_{\delta}^{(2)}=h_{\delta}^{(3)}, e_{\gamma}^{(2)}=e_{\gamma}^{(3)}
\end{array}\right.
$$

and $u^{(2)} \cdot u_{\alpha+\beta}\left(x^{(2)}-c y^{(2)} z^{(2)}\right) \cdot u_{\alpha}\left(z^{(2)}\right) \cdot\left(h_{\beta}^{(2)}-\left(y^{(2)}+y^{(3)}\right) e_{\beta}^{(2)}\right)=0$, i.e.,

$$
u^{(2)} \cdot\left(h_{\beta}^{(2)}+z^{(2)} e_{\alpha}^{(2)}-\left(y^{(2)}+y^{(3)}\right) e_{\beta}^{(2)}-\left(x^{(2)}+c y^{(3)} z^{(2)}\right) e_{\alpha+\beta}^{(2)}\right)=0 .
$$


Finally, $\left.\left.\left(\widetilde{\mathfrak{g}}^{2} \times S_{\alpha}\right)\right|_{(B / B) \times\left(U^{+} B / B\right)^{3}} \subset\left(\widetilde{\mathfrak{g}}^{4}\right)\right|_{(B / B) \times\left(U^{+} B / B\right)^{3}}$ is defined by

$$
u^{(3)}=u^{(4)}, x^{(3)}=x^{(4)}, y^{(3)}=y^{(4)}, h_{\delta}^{(3)}=h_{\delta}^{(4)}, e_{\gamma}^{(3)}=e_{\gamma}^{(4)}
$$

and $u^{(3)} \cdot u_{\alpha+\beta}\left(x^{(3)}\right) \cdot u_{\beta}\left(y^{(3)}\right) \cdot\left(h_{\alpha}^{(3)}-\left(z^{(3)}+z^{(4)}\right) e_{\alpha}^{(3)}\right)=0$, i.e.,

$$
\begin{aligned}
u^{(3)} \cdot\left(h_{\alpha}^{(3)}-\left(z^{(3)}+z^{(4)}\right) e_{\alpha}^{(3)}+\right. & y^{(3)} e_{\beta}^{(3)} \\
& \left.+\left(-x^{(3)}+c y^{(3)}\left(z^{(3)}+z^{(4)}\right)\right) e_{\alpha+\beta}^{(3)}\right)=0 .
\end{aligned}
$$

In each case, the given equations form a regular sequence in $\mathbb{k}\left[\left.\widetilde{\mathfrak{g}}^{4}\right|_{(B / B) \times\left(U^{+} B / B\right)^{3}}\right]$. Let us prove that the union of these equations again forms a regular sequence. First, equations $(*),\left(*^{\prime}\right)$ and $\left(*^{\prime \prime}\right)$ allow us to identify all the coordinates in the fibers (we will thus remove the superscript on them), and to eliminate the coordinates $u^{(j)}$, $x^{(2)}, y^{(2)}, x^{(3)}, z^{(2)}, x^{(4)}, y^{(3)}$. Then equations (2.1.2), (2.1.3) allow us to eliminate $h_{\alpha}$ and $h_{\beta}$, while (2.1.4) becomes $-z^{(4)} e_{\alpha}+y^{(4)} e_{\beta}+c y^{(4)} z^{(4)} e_{\alpha+\beta}=0$, a non-zero equation in the remaining variables. Hence the equations indeed form a regular sequence, and thus the derived tensor product is concentrated in degree 0 .

Moreover, the polynomial $-z^{(4)} e_{\alpha}+y^{(4)} e_{\beta}+c y^{(4)} z^{(4)} e_{\alpha+\beta}$ is irreducible (it is of degree 1 in $e_{\alpha}$, and not divisible by $\left.z^{(4)}\right)$. Hence it defines an integral scheme. Thus the restriction of $\left(S_{\alpha} \times \widetilde{\mathfrak{g}}^{2}\right) \cap\left(\widetilde{\mathfrak{g}} \times S_{\beta} \times \widetilde{\mathfrak{g}}\right) \cap\left(\widetilde{\mathfrak{g}}^{2} \times S_{\alpha}\right)$ to $(B / B) \times\left(U^{+} B / B\right)$ is integral. It follows that the restriction of this scheme to any $B$-translate of $(B / B) \times\left(U^{+} B / B\right)$ is also integral. Hence $\left(S_{\alpha} \times \widetilde{\mathfrak{g}}^{2}\right) \cap\left(\widetilde{\mathfrak{g}} \times S_{\beta} \times \widetilde{\mathfrak{g}}\right) \cap\left(\widetilde{\mathfrak{g}}^{2} \times S_{\alpha}\right)$ is the union of some integral open sets, each one intersecting each other one. Hence it is integral.

2.2. Determination of the image. Now we have to show that

$$
R\left(p_{1,4}\right)_{*}\left(\mathcal{O}_{\left(S_{\alpha} \times \widetilde{\mathfrak{g}}^{2}\right) \cap\left(\widetilde{\mathfrak{g}} \times S_{\beta} \times \widetilde{\mathfrak{g}}\right) \cap\left(\widetilde{\mathfrak{g}}^{2} \times S_{\alpha}\right)}\right)=R\left(p_{1,4}\right)_{*}\left(\mathcal{O}_{\widetilde{\mathcal{Z}}_{\left(s_{\alpha}, s_{\beta}, s_{\alpha}\right)}}\right)
$$

is invariant under the exchange of $\alpha$ and $\beta$. First, as the intersection we consider is reduced, we can work with varieties instead of schemes. In this paragraph we compute the image of $\widetilde{\mathcal{Z}}_{\left(s_{\alpha}, s_{\beta}, s_{\alpha}\right)}$ under $p_{1,4}$, and observe that it is invariant under the exchange of $\alpha$ and $\beta$ (though the variety $\widetilde{\mathcal{Z}}_{\left(s_{\alpha}, s_{\beta}, s_{\alpha}\right)}$ is of course not). Then we show (in 2.4) that $R\left(p_{1,4}\right)_{*}\left(\mathcal{O}_{\widetilde{\mathcal{Z}}_{\left(s_{\alpha}, s_{\beta}, s_{\alpha}\right)}}\right)$ is the sheaf of functions on this image.

So, let us consider $p_{1,4}\left(\widetilde{\mathcal{Z}}_{\left(s_{\alpha}, s_{\beta}, s_{\alpha}\right)}\right)$. It is a closed subvariety of $\widetilde{\mathfrak{g}}^{2}$. Indeed, we have the following diagram, where all the injections are closed immersions:

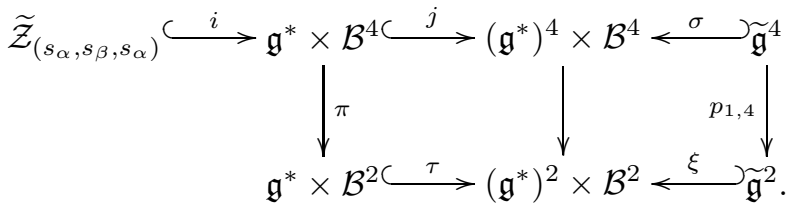

One has $j i\left(\widetilde{\mathcal{Z}}_{\left(s_{\alpha}, s_{\beta}, s_{\alpha}\right)}\right) \subseteq \sigma\left(\widetilde{\mathfrak{g}}^{4}\right)$, and $\xi p_{1,4}\left(\widetilde{\mathcal{Z}}_{\left(s_{\alpha}, s_{\beta}, s_{\alpha}\right)}\right)=\tau \pi i\left(\widetilde{\mathcal{Z}}_{\left(s_{\alpha}, s_{\beta}, s_{\alpha}\right)}\right)$. The morphism $\pi$ being proper, hence closed, the result follows.

Now we compute explicitly $p_{1,4}\left(\widetilde{\mathcal{Z}}_{\left(s_{\alpha}, s_{\beta}, s_{\alpha}\right)}\right)$ as a subset of $\mathfrak{g}^{*} \times \mathcal{B}^{2}$, using the geometric description of $S_{\alpha}$ and $S_{\beta}$ (see 1.4). By $G$-equivariance, we only have to calculate this image over the points $(B / B, w B / B)$ for $w$ in the subgroup of $W$ generated by $s_{\alpha}$ and $s_{\beta}$. Recall that the Demazure resolution $\Phi_{\left(s_{\alpha}, s_{\beta}, s_{\alpha}\right)}$ is an isomorphism over the complement of $\mathcal{X}_{s_{\alpha}}$. Hence if $w=s_{\alpha}^{a_{1}} s_{\beta} s_{\alpha}^{a_{2}}$ with $a_{i} \in$ 
$\{0,1\}$, then for $X \in \mathfrak{g}^{*}$ the point $(X, B / B, w B / B)$ is in the image if and only if $\left(X, B / B, s_{\alpha}^{a_{1}} B / B, s_{\alpha}^{a_{1}} s_{\beta} B / B, s_{\alpha}^{a_{1}} s_{\beta} s_{\alpha}^{a_{2}} B / B\right)$ is in $\widetilde{\mathcal{Z}}_{\left(s_{\alpha}, s_{\beta}, s_{\alpha}\right)}$. Using the geometric description of $S_{\alpha}$, one obtains the condition on $X$ in cases (i) to (iv):

(i) Fiber over $\left(B / B, s_{\alpha} s_{\beta} s_{\alpha} B / B\right): X_{\mid \mathfrak{n} \oplus \mathfrak{k} e_{\alpha} \oplus \mathfrak{k} e_{\beta} \oplus \mathfrak{k} e_{\alpha+\beta}}=0$.

(ii) Fiber over $\left(B / B, s_{\beta} s_{\alpha} B / B\right): X_{\mid \mathfrak{n} \oplus \mathbb{k} h_{\alpha} \oplus \mathbb{k} e_{\beta} \oplus \mathfrak{k} e_{\alpha+\beta}}=0$.

(iii) Fiber over $\left(B / B, s_{\alpha} s_{\beta} B / B\right): X_{\mid \mathfrak{n} \oplus \mathbb{k} h_{\beta} \oplus \mathbb{k} e_{\alpha} \oplus \mathbb{k} e_{\alpha+\beta}}=0$ (observe that $s_{\alpha} s_{\beta}$. $\left.h_{\alpha}=h_{s_{\alpha} s_{\beta}(\alpha)}=h_{\beta}\right)$.

(iv) Fiber over $\left(B / B, s_{\beta} B / B\right): X_{\mid \mathfrak{n} \oplus \mathbb{k} h_{\alpha} \oplus \mathbb{k} h_{\beta} \oplus \mathbb{k} e_{\beta}}=0$.

(v) Fiber over $\left(B / B, s_{\alpha} B / B\right)$ : here the fiber of $\Phi_{\left(s_{\alpha}, s_{\beta}, s_{\alpha}\right)}$ is isomorphic to $\mathbb{P}_{\mathbb{k}}^{1}$, with points the $\left(B / B, g B / B, g B / B, s_{\alpha} B / B\right)$ for $g \in P_{\alpha}$. First, if $g \in s_{\alpha} B$, the condition on $X$ for the point $\left(X, B / B, g B / B, g B / B, s_{\alpha} B / B\right)$ to be in the intersection is $X_{\mid \mathfrak{n} \oplus \mathbb{k} e_{\alpha} \oplus \mathbb{k}\left(s_{\alpha} \cdot h_{\alpha}\right) \oplus \mathbb{k}\left(s_{\alpha} \cdot h_{\beta}\right)}=0$, i.e., $X_{\mid \mathfrak{n} \oplus \mathbb{k} e_{\alpha} \oplus \mathbb{k} h_{\alpha} \oplus \mathbb{k} h_{\beta}}=0$. Second, if $g \notin s_{\alpha} B$, we can assume $g=u_{\alpha}(\epsilon)$ for some $\epsilon \in \mathbb{k}$. Then the corresponding condition on $X$ is to vanish on $\mathfrak{n}$ and on

$$
h_{\alpha}-\epsilon e_{\alpha}, \quad u_{\alpha}(\epsilon) \cdot h_{\beta}=h_{\beta}+\epsilon e_{\alpha} \quad \text { and } \quad e_{\alpha} .
$$

Hence the condition is the same in the two cases. And finally the condition on $X$ for $\left(X, B / B, s_{\beta} B / B\right)$ to be in $p_{1,4}\left(\widetilde{\mathcal{Z}}_{\left(s_{\alpha}, s_{\beta}, s_{\alpha}\right)}\right)$ is

$$
X_{\mid \mathfrak{n} \oplus \mathbb{k} h_{\alpha} \oplus \mathbb{k} h_{\beta} \oplus \mathbb{k} e_{\alpha}}=0 .
$$

(vi) Fiber over $(B / B, B / B)$ : the fiber of $\Phi_{\left(s_{\alpha}, s_{\beta}, s_{\alpha}\right)}$ over $(B / B, B / B)$ is again $\mathbb{P}_{\mathbb{k}}^{1}$, with points the $(B / B, g B / B, g B / B, B / B)$ for $g \in P_{\alpha}$. First, if $g \in s_{\alpha} B / B$, the corresponding condition on $X$ is $X_{\mid \mathfrak{n} \oplus \mathbb{k} e_{\alpha} \oplus \mathbb{k} h_{\alpha+\beta}}=0$. Second, if $g \notin s_{\alpha} B$, then we can assume $g=u_{\alpha}(\epsilon)$ for some $\epsilon \in \mathbb{k}$. The condition on $X$ is then to vanish on $\mathfrak{n}$, on $h_{\alpha}-\epsilon e_{\alpha}$ and on $u_{\alpha}(\epsilon) \cdot h_{\beta}=h_{\beta}+\epsilon e_{\alpha}$. This is equivalent to vanishing on $\mathfrak{n}, h_{\alpha}-\epsilon e_{\alpha}$ and $h_{\alpha}+h_{\beta}=h_{\alpha+\beta}$. Finally, the condition on $X$ for the point $(X, B / B, B / B)$ to be in the image of $\widetilde{\mathcal{Z}}_{\left(s_{\alpha}, s_{\beta}, s_{\alpha}\right)}$ under $p_{1,4}$ is that $X_{\mid \mathfrak{n} \oplus \mathbb{k} h_{\alpha+\beta}}=0$, and that either $X\left(e_{\alpha}\right)=0$, or $X\left(h_{\alpha}-\epsilon e_{\alpha}\right)=0$ for some $\epsilon \in \mathbb{k}$. But if $X\left(e_{\alpha}\right) \neq 0$, then $X\left(h_{\alpha}-\epsilon e_{\alpha}\right)=0$ for $\epsilon=X\left(h_{\alpha}\right) / X\left(e_{\alpha}\right)$. So the condition on $X$ is only

$$
X_{\mid \mathfrak{n} \oplus \mathbb{k} h_{\alpha+\beta}}=0 \text {. }
$$

These considerations show that $p_{1,4}\left(\widetilde{\mathcal{Z}}_{\left(s_{\alpha}, s_{\beta}, s_{\alpha}\right)}\right)$ is a closed subvariety of $\mathfrak{g}^{*} \times$ $\mathcal{B} \times \mathcal{B}$, invariant under the exchange of $\alpha, \beta$ (the computations with $\alpha$ and $\beta$ interchanged are the same, replacing $c$ by $-c)$. We denote it by $S_{\{\alpha, \beta\}}$.

\subsection{Normality of $S_{\{\alpha, \beta\}}$.}

Proposition 2.3.1. The variety $S_{\{\alpha, \beta\}}$ is integral, normal and Cohen-Macaulay.

Proof. 4 First, $S_{\{\alpha, \beta\}}$ is integral because it is the image of $\widetilde{\mathcal{Z}}_{\left(s_{\alpha}, s_{\beta}, s_{\alpha}\right)}$, which is integral by Lemma 2.1.1.

For the other properties, as usual, we only have to consider the situation over $(B / B) \times\left(U^{+} B / B\right)$. We keep the notation of the proof of Lemma 2.1.1 and define $\gamma:=\alpha+\beta$. Recall the isomorphism $U^{+} \cong U_{(\alpha, \beta)}^{+} \times U_{\gamma} \times U_{\beta} \times U_{\alpha}$ (see the proof of Lemma 2.1.1). As $S_{\{\alpha, \beta\}}$ is supported over $\mathcal{B} \times_{G / P_{\alpha, \beta}} \mathcal{B}$, in fact, we only have to consider the situation over $(B / B) \times\left(U_{\gamma} U_{\beta} U_{\alpha} B / B\right) \cong U_{\gamma} \times U_{\beta} \times U_{\alpha}$.

Consider a point

$$
u=u_{\gamma}\left(x_{\gamma}\right) u_{\beta}\left(x_{\beta}\right) u_{\alpha}\left(x_{\alpha}\right) \in U_{\gamma} U_{\beta} U_{\alpha},
$$

\footnotetext{
${ }^{4}$ This proof is due to Patrick Polo.
} 
with $x_{\gamma} x_{\beta} x_{\alpha} \neq 0$ and $x_{\gamma}-c x_{\alpha} x_{\beta} \neq 0$. It can also be written as

$$
u_{\alpha}(x) u_{\beta}(y) u_{\alpha}(z)
$$

with $x_{\gamma}=c x y, x_{\beta}=y, x_{\alpha}=x+z$ (here $\left.x y z \neq 0\right)$. If $X \in(\mathfrak{g} / \mathfrak{n})^{*}$, and $(X, B / B, u B / B)$ is in $S_{\{\alpha, \beta\}}$, then $X$ must vanish on $u_{\alpha}(x) \cdot e_{-\alpha}=e_{-\alpha}+x h_{\alpha}-x^{2} e_{\alpha}$, hence on

$$
h_{\alpha}-x e_{\alpha}
$$

It must also vanish on $u_{\alpha}(x) u_{\beta}(y) \cdot e_{-\beta}$, hence on

$$
h_{\beta}+x e_{\alpha}-y e_{\beta}-c x y e_{\gamma} .
$$

Finally, it must vanish on $u_{\alpha}(x) u_{\beta}(y) u_{\alpha}(z) \cdot e_{-\alpha}$, hence on

$$
(x+z) h_{\alpha}-(x+z)^{2} e_{\alpha}+y z e_{\beta}+c y z(x+z) e_{\alpha+\beta} .
$$

Substracting $(x+z)$ times equation (2.3.2), and dividing by $z$, we obtain that $X$ must vanish on

$$
(x+z) e_{\alpha}-y e_{\beta}-c y(x+z) e_{\gamma} .
$$

The sum of equations (2.3.2) and (2.3.3) becomes

$$
h_{\alpha}+h_{\beta}-x_{\beta} e_{\beta}-x_{\gamma} e_{\gamma} .
$$

Multiplying (2.3.2) by $c x_{\beta}=c y$ gives

$$
c x_{\beta} h_{\alpha}-x_{\gamma} e_{\alpha} .
$$

Equation (2.3.4) can be rewritten as

$$
x_{\alpha} e_{\alpha}-x_{\beta} e_{\beta}-c x_{\alpha} x_{\beta} e_{\gamma} .
$$

Finally, adding $c x_{\alpha}$ times (2.3.2) and $c x$ times (2.3.4) gives

$$
c x_{\alpha} h_{\alpha}-x_{\gamma} e_{\beta}-c x_{\alpha} x_{\gamma} e_{\gamma} .
$$

Let us denote by $M$ the closed subscheme of $\mathbb{A}^{\operatorname{dim}(\mathfrak{g} / \mathfrak{n})+3}$ defined by equations (2.3.5) to (2.3.8). Equation (2.3.5) allows us to eliminate $h_{\beta}$; that is, setting $e=x_{\alpha}$, $f=x_{\beta}, g=x_{\gamma}, h=c h_{\alpha}, i=e_{\alpha}, j=e_{\beta}$ and $k=c e_{\gamma}$, we obtain that the coordinate ring of $M$ is a polynomial ring over $A:=\mathbb{k}[e, f, g, h, i, j, k] /(F, G, H)$, where

$$
\left\{\begin{aligned}
F & =f h-g i, \\
G & =e i-f j-e f k, \\
H & =e h-g j-e g k .
\end{aligned}\right.
$$

Lemma 2.3.9. $A$ is integral, of dimension 5, Cohen-Macaulay and normal. Its singular locus is defined by $e=f=g=h=i=j=0$.

Proof. Let us consider $j^{\prime}:=j+e k$. $A$ is isomorphic to $A^{\prime} \otimes \mathbb{k}[k]$, where

$$
A^{\prime}:=\mathbb{k}\left[e, f, g, h, i, j^{\prime}\right] /\left(f h-g i, e i-f j^{\prime}, e h-g j^{\prime}\right) .
$$

This ring is the algebra of functions on the variety of matrices

$$
\left(\begin{array}{ccc}
h & i & j^{\prime} \\
g & f & e
\end{array}\right)
$$

of rank at most 1 , which is the cone of the Segre embedding of $\mathbb{P}^{1} \times \mathbb{P}^{2}$. This variety is well known to be integral, Cohen-Macaulay and normal, the vertex of the cone (defined by $e=f=g=h=i=j^{\prime}=0$ ) being its unique singularity (see e.g. BV], $2.8,2.11])$. The lemma follows. 
In particular, $M$ is integral. It contains $\left.S_{\{\alpha, \beta\}}\right|_{(B / B) \times\left(U^{+} B / B\right)}$ (the equations are satisfied on a dense open subset of $\left.S_{\{\alpha, \beta\}}\right|_{(B / B) \times\left(U^{+} B / B\right)}$, hence everywhere), which has the same dimension. Hence the two varieties coincide.

We deduce that $S_{\{\alpha, \beta\}}$ is normal and Cohen-Macaulay. This finishes the proof of Proposition 2.3.1,

2.4. End of the proof. We denote by $\Psi_{\left(s_{\alpha}, s_{\beta}, s_{\alpha}\right)}: \widetilde{\mathcal{Z}}_{\left(s_{\alpha}, s_{\beta}, s_{\alpha}\right)} \rightarrow S_{\{\alpha, \beta\}}$ the morphism constructed above (it is the restriction of $p_{1,4}$ ), and similarly with $\alpha$ and $\beta$ interchanged.

Proposition 2.4.1. We have

$$
R\left(\Psi_{\left(s_{\alpha}, s_{\beta}, s_{\alpha}\right)}\right)_{*}\left(\mathcal{O}_{\widetilde{\mathcal{Z}}_{\left(s_{\alpha}, s_{\beta}, s_{\alpha}\right)}}\right) \cong \mathcal{O}_{S_{\{\alpha, \beta\}}}
$$

and similarly with $\alpha$ and $\beta$ interchanged.

Proof. First we prove that $R^{i}\left(\Psi_{\left(s_{\alpha}, s_{\beta}, s_{\alpha}\right)}\right)_{*}\left(\mathcal{O}_{\widetilde{\mathcal{Z}}_{\left(s_{\alpha}, s_{\beta}, s_{\alpha}\right)}}\right)=0$ for $i \geq 1$. The argument for this is adapted from [BK, 3.2.1]. Since the fibers of $\Psi_{\left(s_{\alpha}, s_{\beta}, s_{\alpha}\right)}$ are of dimension at most 1 , by [H2, III.11.2] we have $R^{i}\left(\Psi_{\left(s_{\alpha}, s_{\beta}, s_{\alpha}\right)}\right)_{*}=0$ for $i \geq 2$. Hence we only have to prove the equality $R^{1}\left(\Psi_{\left(s_{\alpha}, s_{\beta}, s_{\alpha}\right)}\right)_{*}\left(\mathcal{O}_{\widetilde{\mathcal{Z}}_{\left(s_{\alpha}, s_{\beta}, s_{\alpha}\right)}}\right)=0$. The following diagram commutes:

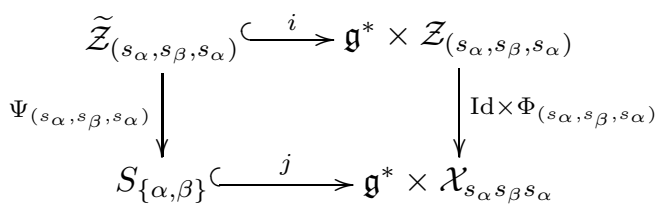

where $i$ and $j$ are closed embeddings. Hence we only have to show that $R^{1}(\mathrm{Id} \times$ $\left.\Phi_{\left(s_{\alpha}, s_{\beta}, s_{\alpha}\right)}\right)_{*}\left(i_{*} \mathcal{O}_{\widetilde{\mathcal{Z}}_{\left(s_{\alpha}, s_{\beta}, s_{\alpha}\right)}}\right)=0$. We have a surjection

$$
\mathcal{O}_{\mathfrak{g}^{*} \times \mathcal{Z}_{\left(s_{\alpha}, s_{\beta}, s_{\alpha}\right)}} \rightarrow i_{*} \mathcal{O}_{\widetilde{\mathcal{Z}}_{\left(s_{\alpha}, s_{\beta}, s_{\alpha}\right)}} .
$$

As $R^{2}\left(\operatorname{Id} \times \Phi_{\left(s_{\alpha}, s_{\beta}, s_{\alpha}\right)}\right)_{*}=0$ (for the same reason as above), we obtain a surjection

$$
R^{1}\left(\operatorname{Id} \times \Phi_{\left(s_{\alpha}, s_{\beta}, s_{\alpha}\right)}\right)_{*}\left(\mathcal{O}_{\left.\mathfrak{g}^{*} \times \mathcal{Z}_{\left(s_{\alpha}, s_{\beta}, s_{\alpha}\right)}\right)}\right) \rightarrow R^{1}\left(\operatorname{Id} \times \Phi_{\left(s_{\alpha}, s_{\beta}, s_{\alpha}\right)}\right)_{*}\left(i_{*} \mathcal{O}_{\widetilde{\mathcal{Z}}_{\left(s_{\alpha}, s_{\beta}, s_{\alpha}\right)}}\right) .
$$

By the classical results on Demazure resolutions, the object on the left hand side is zero. Hence $R^{1}\left(\operatorname{Id} \times \Phi_{\left(s_{\alpha}, s_{\beta}, s_{\alpha}\right)}\right)_{*}\left(i_{*} \mathcal{O}_{\mathcal{Z}_{\left(s_{\alpha}, s_{\beta}, s_{\alpha}\right)}}\right)=0$, as claimed.

Since $\Psi_{\left(s_{\alpha}, s_{\beta}, s_{\alpha}\right)}$ is proper and birational (because $\Phi_{\left(s_{\alpha}, s_{\beta}, s_{\alpha}\right)}$ is), and $S_{\{\alpha, \beta\}}$ is normal (by Proposition 2.3.1), one has $\left(\Psi_{\left(s_{\alpha}, s_{\beta}, s_{\alpha}\right)}\right)_{*}\left(\mathcal{O}_{\widetilde{\mathcal{Z}}_{\left(s_{\alpha}, s_{\beta}, s_{\alpha}\right)}}\right) \cong \mathcal{O}_{S_{\{\alpha, \beta\}}}$ by Zariski's Main Theorem. This proves the result. The assertion with $\alpha$ and $\beta$ interchanged is obtained similarly.

With this proposition the proof of the finite braid relation for the action on $\mathcal{D}^{b} \operatorname{Coh}(\widetilde{\mathfrak{g}})$ (see Theorem 1.4.1) when $\alpha$ and $\beta$ generate a root system of type $\mathbf{A}_{2}$ is complete. 


\section{Finite braid Relations for type $\mathbf{B}_{2}$}

Now we assume that $\alpha$ and $\beta$ generate a root system of type $\mathbf{B}_{2}$. To fix notation, we assume that $\alpha$ is short and $\beta$ is long. Then $\left\langle\alpha, \beta^{\vee}\right\rangle=-1,\left\langle\beta, \alpha^{\vee}\right\rangle=-2$. There exist structure constants $c, d \in \mathbb{k}^{\times}$such that

$$
\forall x, y \in \mathbb{k}, \quad\left(u_{\alpha}(x), u_{\beta}(y)\right)=u_{\alpha+\beta}(c x y) u_{2 \alpha+\beta}\left(d x^{2} y\right)
$$

(again, see [Sp, 8.2.3]). Then, also,

$$
\forall x, y \in \mathbb{k}, \quad\left(u_{\beta}(x), u_{\alpha}(y)\right)=u_{\alpha+\beta}(-c x y) u_{2 \alpha+\beta}\left(-d x y^{2}\right) .
$$

Easy calculations yield the following formulae for the adjoint action of $G$ on $\mathfrak{g}$ :

$$
\begin{array}{ll}
u_{\alpha}(x) \cdot e_{\beta}=e_{\beta}+c x e_{\alpha+\beta}+d x^{2} e_{2 \alpha+\beta}, & u_{\alpha}(x) \cdot h_{\beta}=h_{\beta}+x e_{\alpha}, \\
u_{\alpha}(x) \cdot e_{\alpha+\beta}=e_{\alpha+\beta}+2 \frac{d}{c} x e_{2 \alpha+\beta}, & u_{\beta}(x) \cdot h_{\alpha}=h_{\alpha}+2 x e_{\beta}, \\
u_{\beta}(x) \cdot e_{\alpha}=e_{\alpha}-c x e_{\alpha+\beta}, & u_{\alpha+\beta}(x) \cdot h_{\alpha}=h_{\alpha}, \\
u_{\alpha+\beta}(x) \cdot e_{\alpha}=e_{\alpha}-2 \frac{d}{c} e_{2 \alpha+\beta}, & u_{\alpha+\beta}(x) \cdot h_{\beta}=h_{\beta}-x e_{\alpha+\beta}, \\
u_{\alpha+\beta}(x) \cdot e_{-\alpha}=e_{-\alpha}-\frac{2}{c} x e_{\beta}, & u_{2 \alpha+\beta}(x) \cdot h_{\alpha}=h_{\alpha}-2 x e_{2 \alpha+\beta}, \\
u_{\alpha+\beta}(x) \cdot e_{-\beta}=e_{-\beta}+\frac{1}{c} x e_{\alpha}-\frac{d}{c^{2}} x^{2} e_{2 \alpha+\beta}, & \\
u_{2 \alpha+\beta}(x) \cdot e_{-\alpha}=e_{-\alpha}-\frac{2 c}{d} x e_{\alpha+\beta} . &
\end{array}
$$

We also have $h_{\alpha+\beta}=h_{\alpha}+2 h_{\beta}, h_{2 \alpha+\beta}=h_{\alpha}+h_{\beta}$.

In this section we prove the finite braid relation for the simple roots $\alpha$ and $\beta$. The proof is very similar to the one in the previous section. We assume throughout the section that $\operatorname{char}(\mathbb{k}) \neq 2$.

\subsection{Derived tensor product.}

Lemma 3.1.1. There exist isomorphisms

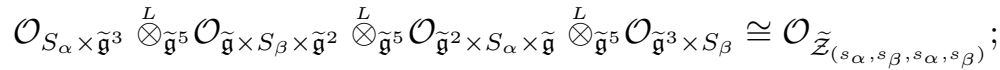

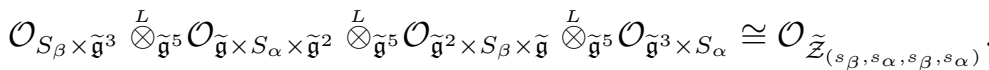

Moreover, the varieties $\widetilde{\mathcal{Z}}_{\left(s_{\alpha}, s_{\beta}, s_{\alpha}, s_{\beta}\right)}$ and $\widetilde{\mathcal{Z}}_{\left(s_{\beta}, s_{\alpha}, s_{\beta}, s_{\alpha}\right)}$ are integral.

Proof. As for Lemma2.1.1, we prove the result in the first case only, by computation of equations (the second case can be treated similarly). Let us choose an ordering of $R^{+}$such that the last four roots are $2 \alpha+\beta, \alpha+\beta, \beta, \alpha$ (in this order). Let $U_{(\alpha)}^{+}, U_{(\beta)}^{+}, U_{(\alpha, \beta)}^{+}$be the product of the $U_{\gamma}$ for $\gamma \in R^{+}-\{\alpha\}, \gamma \in R^{+}-\{\beta\}$, $\gamma \in R^{+}-\{\alpha, \beta, \alpha+\beta, 2 \alpha+\beta\}$. Under the isomorphism $U^{+} \cong \prod_{\gamma \in R^{+}} U_{\gamma}$, the restriction to $U^{+}$of the projections $\pi_{\alpha}: \mathcal{B} \rightarrow \mathcal{P}_{\alpha}, \pi_{\beta}: \mathcal{B} \rightarrow \mathcal{P}_{\beta}$ become the morphisms $U_{(\alpha)}^{+} \times U_{\alpha} \rightarrow U_{(\alpha)}^{+}$and

$\left\{\begin{array}{ccc}U_{(\alpha, \beta)}^{+} \times U_{2 \alpha+\beta} \times U_{\alpha+\beta} \times U_{\beta} \times U_{\alpha} & \rightarrow & U_{(\alpha, \beta)}^{+} \times U_{2 \alpha+\beta} \times U_{\alpha+\beta} \times U_{\alpha} \\ \left(u, u_{2 \alpha+\beta}(x), u_{\alpha+\beta}(y), u_{\beta}(z), u_{\alpha}(t)\right) & \mapsto & \left(u, u_{2 \alpha+\beta}\left(x-d t^{2} z\right), u_{\alpha+\beta}(y-c z t), u_{\alpha}(t)\right) .\end{array}\right.$

As coordinates we will use the $u^{(j)}, x^{(j)}, y^{(j)}, z^{(j)}$ and $t^{(j)}$ on the base $(j=2, \ldots, 5)$, and $e_{\gamma}^{(j)}\left(\gamma \in R^{+}\right), h_{\delta}^{(j)}(\delta \in \Phi)$ in the fibers $(j=1, \ldots, 5)$.

In these coordinates, $\left.\left.\left(S_{\alpha} \times \widetilde{\mathfrak{g}}^{3}\right)\right|_{(B / B) \times\left(U^{+} B / B\right)^{4}} \subset\left(\tilde{\mathfrak{g}}^{5}\right)\right|_{(B / B) \times\left(U^{+} B / B\right)^{4}}$ is defined by the equations

$$
u^{(2)}=1, x^{(2)}=0, y^{(2)}=0, z^{(2)}=0, e_{\gamma}^{(1)}=e_{\gamma}^{(2)}, h_{\delta}^{(1)}=h_{\delta}^{(2)}
$$


for $\delta \in \Phi, \gamma \in R^{+}$, and

$$
h_{\alpha}^{(1)}-t^{(2)} e_{\alpha}^{(1)}=0 .
$$

Similarly, $\left.\left.\left(\tilde{\mathfrak{g}} \times S_{\beta} \times \widetilde{\mathfrak{g}}^{2}\right)\right|_{(B / B) \times\left(U^{+} B / B\right)^{4}} \subset\left(\tilde{\mathfrak{g}}^{5}\right)\right|_{(B / B) \times\left(U^{+} B / B\right)^{4}}$ is defined by the equations

$$
\left\{\begin{array}{l}
u^{(2)}=u^{(3)}, x^{(2)}-d\left(t^{(2)}\right)^{2} z^{(2)}=x^{(3)}-d\left(t^{(3)}\right)^{2} z^{(3)}, t^{(2)}=t^{(3)}, \\
y^{(2)}-c z^{(2)} t^{(2)}=y^{(3)}-c z^{(3)} t^{(3)}, e_{\gamma}^{(2)}=e_{\gamma}^{(3)}, h_{\delta}^{(2)}=h_{\delta}^{(3)}
\end{array}\right.
$$

and $u^{(2)} \cdot u_{2 \alpha+\beta}\left(x^{(2)}-d\left(t^{(2)}\right)^{2} z^{(2)}\right) \cdot u_{\alpha+\beta}\left(y^{(2)}-c z^{(2)} t^{(2)}\right) \cdot u_{\alpha}\left(t^{(2)}\right) \cdot\left(h_{\beta}^{(2)}-\left(z^{(2)}+\right.\right.$ $\left.\left.z^{(3)}\right) e_{\beta}^{(2)}\right)=0$, i.e.,

$$
\begin{aligned}
u^{(2)} \cdot\left(h_{\beta}^{(2)}+t^{(2)} e_{\alpha}^{(2)}-\right. & \left(z^{(2)}+z^{(3)}\right) e_{\beta}^{(2)}+\left(-y^{(2)}-c t^{(2)} z^{(3)}\right) e_{\alpha+\beta}^{(2)} \\
+ & \left.\left(-2 \frac{d}{c} y^{(2)} t^{(2)}+d\left(t^{(2)}\right)^{2}\left(z^{(2)}-z^{(3)}\right)\right) e_{2 \alpha+\beta}^{(2)}\right)=0 .
\end{aligned}
$$

Next, $\left.\left.\left(\widetilde{\mathfrak{g}}^{2} \times S_{\alpha} \times \tilde{\mathfrak{g}}\right)\right|_{(B / B) \times\left(U^{+} B / B\right)^{4}} \subset\left(\widetilde{\mathfrak{g}}^{5}\right)\right|_{(B / B) \times\left(U^{+} B / B\right)^{4}}$ is defined by the equations

$$
\left\{\begin{array}{l}
u^{(3)}=u^{(4)}, x^{(3)}=x^{(4)}, y^{(3)}=y^{(4)}, \\
z^{(3)}=z^{(4)}, e_{\gamma}^{(3)}=e_{\gamma}^{(4)}, h_{\delta}^{(3)}=h_{\delta}^{(4)}
\end{array}\right.
$$

and $u^{(3)} \cdot u_{2 \alpha+\beta}\left(x^{(3)}\right) \cdot u_{\alpha+\beta}\left(y^{(3)}\right) \cdot u_{\beta}\left(z^{(3)}\right) \cdot\left(h_{\alpha}^{(3)}-\left(t^{(3)}+t^{(4)}\right) e_{\alpha}^{(3)}\right)=0$, i.e.,

$$
\begin{aligned}
u^{(3)} \cdot\left(h_{\alpha}^{(3)}-\left(t^{(3)}+t^{(4)}\right) e_{\alpha}^{(3)}\right. & +2 z^{(3)} e_{\beta}^{(3)}+c z^{(3)}\left(t^{(3)}+t^{(4)}\right) e_{\alpha+\beta}^{(3)} \\
& \left.+\left(-2 x^{(3)}+2 \frac{d}{c} y^{(3)}\left(t^{(3)}+t^{(4)}\right) e_{2 \alpha+\beta}\right)\right)=0 .
\end{aligned}
$$

Finally, $\left.\left.\left(\widetilde{\mathfrak{g}}^{3} \times S_{\beta}\right)\right|_{(B / B) \times\left(U^{+} B / B\right)^{4}} \subset\left(\widetilde{\mathfrak{g}}^{5}\right)\right|_{(B / B) \times\left(U^{+} B / B\right)^{4}}$ is defined by the equations

$\left(*^{\prime \prime \prime}\right) \quad\left\{\begin{array}{l}u^{(4)}=u^{(5)}, x^{(4)}-d\left(t^{(4)}\right)^{2} z^{(4)}=x^{(5)}-d\left(t^{(5)}\right)^{2} z^{(5)}, t^{(4)}=t^{(5)}, \\ y^{(4)}-c z^{(4)} t^{(4)}=y^{(5)}-c z^{(5)} t^{(5)}, e_{\gamma}^{(4)}=e_{\gamma}^{(5)}, h_{\delta}^{(4)}=h_{\delta}^{(5)},\end{array}\right.$

and $u^{(4)} \cdot u_{2 \alpha+\beta}\left(x^{(4)}-d\left(t^{(4)}\right)^{2} z^{(4)}\right) \cdot u_{\alpha+\beta}\left(y^{(4)}-c z^{(4)} t^{(4)}\right) \cdot u_{\alpha}\left(t^{(4)}\right) \cdot\left(h_{\beta}^{(4)}-\left(z^{(4)}+\right.\right.$ $\left.\left.z^{(5)}\right) e_{\beta}^{(4)}\right)=0$, i.e.,

$$
\begin{aligned}
u^{(4)} \cdot\left(h_{\beta}^{(4)}+t^{(4)} e_{\alpha}^{(4)}-\right. & \left(z^{(4)}+z^{(5)}\right) e_{\beta}^{(4)}+\left(-y^{(4)}-c t^{(4)} z^{(5)}\right) e_{\alpha+\beta}^{(4)} \\
+ & \left.\left(-2 \frac{d}{c} y^{(4)} t^{(4)}+d\left(t^{(4)}\right)^{2}\left(z^{(4)}-z^{(5)}\right)\right) e_{2 \alpha+\beta}^{(4)}\right)=0 .
\end{aligned}
$$

As in the proof of Lemma 2.1.1 we have to show that the union of these equations forms a regular sequence. The equations $(*)$ to $\left(*^{\prime \prime \prime}\right)$ allow us to eliminate the coordinates $u^{(j)}, x^{(2)}, y^{(2)}, z^{(2)}, x^{(3)}, y^{(3)}, t^{(3)}, x^{(4)}, y^{(4)}, z^{(4)}, x^{(5)}, y^{(5)}, t^{(5)}$, and to identify the coordinates in the fibers, which we will denote by $e_{\gamma}$ and $h_{\delta}$. Then, equations (3.1.2) and (3.1.3) allow us to eliminate $h_{\alpha}$ and $h_{\beta}$. With these simplifications, equations (3.1.4) and (3.1.5) become

$$
\begin{gathered}
-t^{(4)} e_{\alpha}+2 z^{(3)} e_{\beta}+c z^{(3)}\left(t^{(2)}+t^{(4)}\right) e_{\alpha+\beta}+2 d z^{(3)} t^{(2)} t^{(4)} e_{2 \alpha+\beta}=0, \\
\left(t^{(4)}-t^{(2)}\right) e_{\alpha}-z^{(5)} e_{\beta}-c t^{(4)} z^{(5)} e_{\alpha+\beta} \\
+d\left(z^{(3)}\left(t^{(2)}-t^{(4)}\right)^{2}-\left(t^{(4)}\right)^{2} z^{(5)}\right) e_{2 \alpha+\beta}=0 .
\end{gathered}
$$


Let us denote by $P$ the polynomial of (3.1.6), and by $Q$ the polynomial of (3.1.7). Then $P$ and $Q$ are irreducible and distinct. Hence they form a regular sequence in $\mathbb{k}\left[z^{(3)}, z^{(5)}, t^{(2)}, t^{(4)}, e_{\gamma}, \gamma \in R^{+}, h_{\delta}, \delta \in \Phi-\{\alpha, \beta\}\right]$. This proves that the tensor product we are considering is indeed concentrated in degree 0 , and that the ring $\mathbb{k}\left[z^{(3)}, z^{(5)}, t^{(2)}, t^{(4)}, e_{\gamma}, h_{\delta}\right] /(P, Q)$ is Cohen-Macaulay (see $[\mathrm{BH}, 2.1 .3]$ ). We prove in the next lemma that this ring is an integral domain. We deduce, as in the case of $\mathbf{A}_{2}$, that $\widetilde{\mathcal{Z}}_{\left(s_{\alpha}, s_{\beta}, s_{\alpha}, s_{\beta}\right)}$ is an integral scheme.

Lemma 3.1.8. $\mathbb{k}\left[z^{(3)}, z^{(5)}, t^{(2)}, t^{(4)}, e_{\gamma}, h_{\delta}\right] /(P, Q)$ is an integral domain.

Proof. First, let us prove that the closed subvariety $N$ of $\mathbb{k}^{\operatorname{dim}(\mathfrak{g} / \mathfrak{n})+2}$ defined by $P$ and $Q$ is irreducible. The restriction of this subvariety to the open set defined by $t^{(4)} \neq 0$ is irreducible (indeed, on this open set $P$ gives $e_{\alpha}$ as a polynomial in the other coordinates and $\left(t^{(4)}\right)^{-1}$, and replacing in $Q$ we still obtain an irreducible polynomial). Similarly, for the intersections with the open set defined by $z^{(3)} \neq 0$, and with the open set defined by $z^{(5)} \neq 0$. Now $N$ is isomorphic to the closure of its intersection with the open set $\left\{t^{(4)} \neq 0\right\} \cup\left\{z^{(3)} \neq 0\right\} \cup\left\{z^{(5)} \neq 0\right\}$ (indeed, if $t^{(4)}=z^{(3)}=0, P$ is zero, and $Q=-t^{(2)} e_{\alpha}-z^{(5)} e_{\beta}$ is an irreducible polynomial, whose variety of zeros intersect the open set $\left\{z^{(5)} \neq 0\right\}$ ). This intersection is irreducible (it is the union of three irreducible open sets, each one intersecting each other one). Hence $N$ is irreducible.

Now we have to show that the ring $\mathbb{k}\left[z^{(3)}, z^{(5)}, t^{(2)}, t^{(4)}, e_{\gamma}, h_{\delta}\right] /(P, Q)$ is reduced, i.e., that it satisfies properties $\left(R_{0}\right)$ and $\left(S_{1}\right)$ (see [Ma, p. 125]). As we have seen that it is Cohen-Macaulay, and that the corresponding scheme is irreducible, we only have to prove that it is regular at some point. But it is clearly regular at the point defined by $t^{(2)}=t^{(4)}=1, z^{(3)}=0, z^{(5)}=1, e_{\alpha}=e_{\beta}=e_{\alpha+\beta}=e_{2 \alpha+\beta}=0$ (consider the partial diveratives of $P$ and $Q$ with respect to $e_{\alpha}$ and $e_{\beta}$ ).

3.2. Determination of the image. As in 2.2, we have to identify the images of $\widetilde{\mathcal{Z}}_{\left(s_{\alpha}, s_{\beta}, s_{\alpha}, s_{\beta}\right)}$ and $\widetilde{\mathcal{Z}}_{\left(s_{\beta}, s_{\alpha}, s_{\beta}, s_{\alpha}\right)}$ under $p_{1,5}: \widetilde{\mathfrak{g}}^{5} \rightarrow \widetilde{\mathfrak{g}}^{2}$ (these are closed subvarieties of $\widetilde{\mathfrak{g}}^{2}$ ), and observe that they coincide. We only indicate the computations for the first case, the second one being similar. By $G$-equivariance we only have to compute the fibers of this image over the points $(B / B, w B / B)$ for $w$ in the subgroup of $W$ generated by $s_{\alpha}$ and $s_{\beta}$. In this case the Demazure resolution $\Phi_{\left(s_{\alpha}, s_{\beta}, s_{\alpha}, s_{\beta}\right)}$ is an isomorphism over the complement of $\mathcal{X}_{s_{\alpha} s_{\beta}}$. This gives the condition on $X \in \mathfrak{g}^{*}$ for the point $(X, B / B, w B / B)$ to be in $p_{1,5}\left(\widetilde{\mathcal{Z}}_{\left(s_{\alpha}, s_{\beta}, s_{\alpha}, s_{\beta}\right)}\right)$ in cases (i) to (iv).

(i) Fiber over $\left(B / B, s_{\alpha} s_{\beta} s_{\alpha} s_{\beta} B / B\right): X_{\mid \mathfrak{n} \oplus \mathbb{k} e_{\alpha} \oplus \mathbb{k} e_{\beta} \oplus \mathbb{k} e_{\alpha+\beta} \oplus \mathbb{k} e_{2 \alpha+\beta}}=0$.

(ii) Fiber over $\left(B / B, s_{\alpha} s_{\beta} s_{\alpha} B / B\right): X_{\mid \mathfrak{n} \oplus \mathbb{k} e_{\alpha} \oplus \mathbb{k} e_{\alpha+\beta} \oplus \mathbb{k} e_{2 \alpha+\beta} \oplus \mathbb{k} h_{\beta}}=0$.

(iii) Fiber over $\left(B / B, s_{\beta} s_{\alpha} s_{\beta} B / B\right): X_{\mid \mathfrak{n} \oplus \mathbb{k} e_{\beta} \oplus \mathbb{k} e_{\alpha+\beta} \oplus \mathbb{k} e_{2 \alpha+\beta} \oplus \mathbb{k} h_{\alpha}}=0$.

(iv) Fiber over $\left(B / B, s_{\beta} s_{\alpha} B / B\right): X_{\mid \mathfrak{n} \oplus \mathbb{k} e_{\beta} \oplus \mathbb{k} e_{\alpha+\beta} \oplus \mathbb{k} h_{\alpha} \oplus \mathbb{k} h_{\beta}}=0$.

(v) Fiber over $\left(B / B, s_{\alpha} s_{\beta} B / B\right)$ : The fiber of $\Phi_{\left(s_{\alpha}, s_{\beta}, s_{\alpha}, s_{\beta}\right)}$ is isomorphic to two copies of $\mathbb{P}_{\mathbb{k}}^{1}$ with one common point. Its elements are, on the one hand, the $\left(B / B, s_{\alpha} B / B, s_{\alpha} g B / B, s_{\alpha} g B / B, s_{\alpha} s_{\beta} B / B\right)$ for $g \in P_{\beta}$ and, on the other hand, the $\left(B / B, g B / B, g B / B, s_{\alpha} B / B, s_{\alpha} s_{\beta} B / B\right)$ for $g \in P_{\alpha}$. One verifies that the conditions on $X$ corresponding to each of these points are the same, namely

$$
X_{\mid \mathfrak{n} \oplus \mathbb{k} e_{\alpha} \oplus \mathbb{k} e_{2 \alpha+\beta} \oplus \mathbb{k} h_{\alpha} \oplus \mathbb{k} h_{\beta}}=0 .
$$

(vi) Fiber over $\left(B / B, s_{\alpha} B / B\right)$ : The fiber of the Demazure resolution is formed by the points $\left(B / B, g B / B, g B / B, s_{\alpha} B / B, s_{\alpha} B / B\right)$ for $g \in P_{\alpha}$ and the points 
$\left(B / B, s_{\alpha} B / B, s_{\alpha} g B / B, s_{\alpha} g B / B, s_{\alpha} B / B\right)$ for $g \in P_{\beta}$. Let us compute the conditions on $X$ corresponding to the each of these points. We begin with the points $\left(B / B, g B / B, g B / B, s_{\alpha} B / B, s_{\alpha} B / B\right)$ for $g \in P_{\alpha}$. If $g \in s_{\alpha} B$, the condition is to vanish on $\mathfrak{n}, e_{\alpha}, s_{\alpha} \cdot h_{\beta}=h_{\alpha}+h_{\beta}$ and $s_{\alpha} \cdot h_{\alpha}=-h_{\alpha}$, i.e., $X_{\mid \mathfrak{n} \oplus \mathbb{k} e_{\alpha} \oplus \mathbb{k} h_{\alpha} \oplus \mathbb{k} h_{\beta}}=0$. If $g=u_{\alpha}(\epsilon)$ for some $\epsilon \in \mathbb{k}$, then the condition is to vanish on $h_{\alpha}-\epsilon e_{\alpha}, u_{\alpha}(\epsilon) \cdot h_{\beta}=$ $h_{\beta}+\epsilon e_{\alpha}, e_{\alpha}$ and $s_{\alpha} \cdot h_{\beta}=h_{\alpha}+h_{\beta}$, i.e., the same condition. Now, let us consider the points $\left(B / B, s_{\alpha} B / B, s_{\alpha} g B / B, s_{\alpha} g B / B, s_{\alpha} B / B\right)$ for $g \in P_{\beta}$. If $g \in s_{\beta} B$, then the condition is to vanish on $e_{\alpha}, e_{2 \alpha+\beta}$ and $s_{\alpha} s_{\beta} \cdot h_{\alpha}=h_{\alpha+\beta}$. If $g=u_{\beta}(\epsilon)$, the condition is to vanish on $e_{\alpha}, s_{\alpha} \cdot\left(h_{\beta}-\epsilon e_{\beta}\right)$ and $s_{\alpha} u_{\beta}(\epsilon) \cdot h_{\alpha}=s_{\alpha} \cdot\left(h_{\alpha}+2 \epsilon e_{\beta}\right)$, i.e., on $e_{\alpha}, s_{\alpha} \cdot\left(h_{\beta}-\epsilon e_{\beta}\right)$ and $s_{\alpha} \cdot\left(h_{\alpha}+2 h_{\beta}\right)=s_{\alpha} \cdot h_{\alpha+\beta}=h_{\alpha+\beta}$. As in 2.2 (vi), the condition on $X$ for the point $\left(X, B / B, s_{\alpha} B / B\right)$ to be in the image of $p_{1,5}$ is finally

$$
X_{\mid \mathfrak{n} \oplus \mathbb{k} e_{\alpha} \oplus \mathbb{k} h_{\alpha+\beta}}=0 \text {. }
$$

(vii) Fiber over $\left(B / B, s_{\beta} B / B\right)$ : Similarly, the condition is

$$
X_{\mid \mathfrak{n} \oplus \mathbb{k} e_{\beta} \oplus \mathbb{k} h_{2 \alpha+\beta}}=0 \text {. }
$$

(viii) Fiber over $(B / B, B / B)$ : The fiber of the Demazure resolution is given on the one hand by the $(B / B, g B / B, g B / B, B / B, B / B)$ for $g \in P_{\alpha}$ and on the other hand by the $(B / B, B / B, g B / B, g B / B, B / B)$ for $g \in P_{\beta}$. In the first case, if $g \in B$, then the corresponding condition of $X$ is to vanish on $\mathfrak{n}, h_{\alpha}$ and $h_{\beta}$. If $g \notin B$, then the condition is to vanish on $\mathfrak{n}, e_{\alpha}, h_{\alpha}$ and $h_{\beta}$. The situation is similar in the second case. Hence the condition on $X$ for $(X, B / B, B / B)$ to be in the image is

$$
X_{\mid \mathfrak{n} \oplus \mathbb{k} h_{\alpha} \oplus \mathbb{k} h_{\beta}}=0 .
$$

It follows from these computations and the similar ones with $\alpha$ and $\beta$ interchanged (computing $p_{1,5}\left(\widetilde{\mathcal{Z}}_{\left(s_{\beta}, s_{\alpha}, s_{\beta}, s_{\alpha}\right)}\right)$ instead of $p_{1,5}\left(\widetilde{\mathcal{Z}}_{\left(s_{\alpha}, s_{\beta}, s_{\alpha}, s_{\beta}\right)}\right)$ amounts to replacing $\alpha$ by $\beta, \beta$ by $\alpha, \alpha+\beta$ by $\beta+2 \alpha$, and $\beta+2 \alpha$ by $\alpha+\beta)$ that the images under $p_{1,5}$ of $\widetilde{\mathcal{Z}}_{\left(s_{\alpha}, s_{\beta}, s_{\alpha}, s_{\beta}\right)}$ and $\widetilde{\mathcal{Z}}_{\left(s_{\beta}, s_{\alpha}, s_{\beta}, s_{\alpha}\right)}$ coincide. We let $S_{\{\alpha, \beta\}}$ be this image.

\subsection{Normality of $S_{\{\alpha, \beta\}}$ •}

Proposition 3.3.1. The variety $S_{\{\alpha, \beta\}}$ is integral and normal.

Proof.5 Let us define $\gamma:=\alpha+\beta, \delta:=2 \alpha+\beta$. As for type $\mathbf{A}_{2}$, we already know that $S_{\{\alpha, \beta\}}$ is integral, and we only have to consider the situation over $(B / B) \times$ $\left(U_{\delta} U_{\gamma} U_{\beta} U_{\alpha} B / B\right)$. In this proof we consider $S_{\{\alpha, \beta\}}$ as the image of $\widetilde{\mathcal{Z}}_{\left(s_{\beta}, s_{\alpha}, s_{\beta}, s_{\alpha}\right)}$.

Let

$$
u=u_{\delta}\left(x_{\delta}\right) u_{\gamma}\left(x_{\gamma}\right) u_{\beta}\left(x_{\beta}\right) u_{\alpha}\left(x_{\alpha}\right) \in U_{\delta} U_{\gamma} U_{\beta} U_{\alpha},
$$

with $x_{\alpha} x_{\beta} x_{\gamma} x_{\delta} \neq 0, x_{\gamma} x_{\beta}-\frac{d}{c^{2}} x_{\gamma}^{2} \neq 0$ and $x_{\alpha} x_{\gamma}-\frac{c}{d} x_{\delta} \neq 0$. We have

$$
u=u_{\beta}(t) u_{\alpha}(z) u_{\beta}(y) u_{\alpha}(x)
$$

with $x_{\alpha}=x+z, x_{\beta}=y+t, x_{\gamma}=c y z, x_{\delta}=d y z^{2}$ (here $x y z t \neq 0$ ). Then if $(X, B / B, u B / B)$ is in $S_{\{\alpha, \beta\}}, X$ must vanish on

$$
h_{\beta}-t e_{\beta} .
$$

It also vanishes on $u_{\beta}(t) u_{\alpha}(z) \cdot e_{-\alpha}$, hence on $h_{\alpha}+2 t e_{\beta}-z e_{\alpha}+c z t e_{\gamma}$. Adding two times (3.3.2), one obtains

$$
h_{\gamma}-z e_{\alpha}+c z t e_{\gamma} .
$$

\footnotetext{
${ }^{5}$ This proof is a simplification of an earlier one due to Patrick Polo.
} 
Further, $X$ must vanish on $u_{\beta}(t) u_{\alpha}(z) u_{\beta}(y) \cdot e_{-\beta}$, hence on $u_{\beta}(t) u_{\alpha}(z) \cdot\left(h_{\beta}-y e_{\beta}\right)$. Substracting (3.3.2), one obtains

$$
z e_{\alpha}-(y+t) e_{\beta}-c z(y+t) e_{\gamma}-d y z^{2} e_{\delta} .
$$

Finally, $X$ vanishes on $u_{\beta}(t) u_{\alpha}(z) u_{\beta}(y) u_{\alpha}(x) \cdot e_{-\alpha}$, hence on $u_{\beta}(t) u_{\alpha}(z) u_{\beta}(y) \cdot\left(h_{\alpha}-\right.$ $\left.x e_{\alpha}\right)$. Substracting $u_{\beta}(t) \cdot\left(h_{\alpha}-z e_{\alpha}\right)$, one obtains

$$
-(x+z) e_{\alpha}+2 y e_{\beta}+c((y+t)(x+z)+y z) e_{\gamma}+2 d y z(x+z) e_{\delta} .
$$

Let us transform our equations (3.3.2) to (3.3.5) to obtain equations in $x_{\alpha}, x_{\beta}$, $x_{\gamma}, x_{\delta}$. Substracting (3.3.5) from two times (3.3.2), one obtains

$$
2 h_{\beta}+x_{\alpha} e_{\alpha}-2 x_{\beta} e_{\beta}-\left(c x_{\alpha} x_{\beta}+x_{\gamma}\right) e_{\gamma}-\frac{2 d}{c} x_{\alpha} x_{\gamma} e_{\delta} .
$$

Similarly, adding (3.3.3) and (3.3.4), one obtains

$$
h_{\gamma}-x_{\beta} e_{\beta}-x_{\gamma} e_{\gamma}-x_{\delta} e_{\delta} .
$$

Then, one verifies that $(x+z)$ times (3.3.4) plus $z$ times (3.3.5), and $2 y$ times (3.3.4) plus $v$ times (3.3.5) give respectively

$$
\begin{gathered}
\left(\frac{2}{c} x_{\gamma}-x_{\alpha} x_{\beta}\right) e_{\beta}+\frac{c}{d} x_{\delta} e_{\gamma}+x_{\alpha} x_{\delta} e_{\delta} \\
\left(\frac{2}{c} x_{\gamma}-x_{\alpha} x_{\beta}\right) e_{\alpha}+x_{\beta}\left(c x_{\alpha} x_{\beta}-x_{\gamma}\right) e_{\gamma}+\frac{2 d}{c} x_{\gamma}\left(x_{\alpha} x_{\beta}-\frac{1}{c} x_{\gamma}\right) e_{\delta} .
\end{gathered}
$$

Finally, $x_{\gamma}$ times (3.3.4) gives

$$
\frac{c}{d} x_{\delta} e_{\alpha}-x_{\beta} x_{\gamma} e_{\beta}-\frac{c^{2}}{d} x_{\beta} x_{\delta} e_{\gamma}-x_{\gamma} x_{\delta} e_{\delta}
$$

Equations (3.3.6) and (3.3.7) express $h_{\beta}, h_{\gamma}$ in terms of the other variables. We denote by $E, F$ and $G$ the polynomials of (3.3.8), (3.3.9) and (3.3.10).

Now we can finish the proof exactly as in the case of $\mathbf{A}_{2}$. In the next lemma we show that the scheme defined by $E, F$ and $G$ is normal and integral. Moreover, it contains $\left.S_{\{\alpha, \beta\}}\right|_{(B / B) \times\left(U^{+} B / B\right)}$ as a closed subvariety, and has the same dimension. Hence the two varieties coincide.

Lemma 3.3.11. The ring

$$
A:=\mathbb{k}\left[x_{\alpha}, x_{\beta}, x_{\gamma}, x_{\delta}, e_{\alpha}, e_{\beta}, e_{\gamma}, e_{\delta}\right] /(E, F, G)
$$

is a normal domain.

Proof. Let us forget about the previous notation $x, y, z$ and $t$. Now we define $x=x_{\alpha}, y=-x_{\beta}, z=\frac{2}{c} x_{\gamma}-x_{\alpha} x_{\beta}, t=-x_{\delta}, f=\frac{2}{c}\left(\frac{c}{d} e_{\alpha}-\frac{c^{2}}{d} x_{\beta} e_{\gamma}-x_{\gamma} e_{\delta}\right), g=e_{\beta}$, $h=\frac{c}{d} e_{\gamma}+x_{\alpha} e_{\delta}, i=e_{\delta}$. Then we have $A \cong A^{\prime}[i]$, where

$$
A^{\prime}:=\mathbb{k}[x, y, z, t, f, g, h] /(z g-t h, z f-(z-x y) h, y(z-x y) g-t f) .
$$

Let us first show that the closed subvariety of $\mathbb{A}^{7}$ corresponding to $A^{\prime}$, denoted by $M$, is irreducible. The restriction of $M$ to the open set $\{t \neq 0\}$ is defined by the equations $h=z g / t$ and $f=y(z-x y) g / t$. Hence it is irreducible. Similarly, for the open sets $\{z \neq 0\}$ and $\{f \neq 0\}$. These open sets intersect each other in $M$. Hence the restriction of $M$ to $\{t \neq 0\} \cup\{z \neq 0\} \cup\{f \neq 0\}$ is also irreducible. As $M$ is the closure of this restriction (indeed, if $z=t=0$, the condition $(x, y, z, t, f, g, h) \in M$ does not depend on $f$ ), it is irreducible. 
Now we show that $A$ is normal (hence also reduced). We will use the following lemma (see [BV, 16.24]):

Lemma 3.3.12. Let $S$ be a noetherian ring, and $y \in S$ which is not a zero divisor. Assume that $S /(y)$ is reduced and $S\left[y^{-1}\right]$ is normal. Then $S$ is normal.

Let us apply the lemma to $S=A^{\prime}$ and our element $y$. It is clear that $y$ is not nilpotent (it is not zero on $M$ ). Since $M$ is irreducible, $y$ is not a zero-divisor. Now $A^{\prime} /(y)$ is isomorphic to

$$
\mathbb{k}[x, z, t, f, g, h] / I
$$

where $I=(z g-t h, z f, f t)$. This ideal is the intersection of the prime ideals $(z, t)$ and $(f, z g-t h)$ of $\mathbb{k}[x, z, t, f, g, h]$, hence it is reduced.

Consider the ring $A^{\prime}\left[y^{-1}\right]$. Using the change of coordinates $f^{\prime}=f /\left(y^{2}\right)$ and $x^{\prime}=-x+(z / y)$, it is isomorphic to

$$
\left(\mathbb{k}\left[x^{\prime}, z, t, f^{\prime}, g, h\right] /\left(z g-t h, x^{\prime} g-f^{\prime} t, z f^{\prime}-h x^{\prime}\right)\right)\left[y, y^{-1}\right] .
$$

As in the proof of Lemma 2.3.9, this ring is normal. This concludes the proof of Lemma 3.3.11.

Remark 3.3.13. As in type $\mathbf{A}_{2}$, one can show that $S_{\{\alpha, \beta\}}$ is Cohen-Macaulay. As our proof is long and not needed here, we omit it.

3.4. End of the proof. Now, exactly as in Proposition 2.4.1 one proves that

$$
R\left(\Psi_{\left(s_{\alpha}, s_{\beta}, s_{\alpha}, s_{\beta}\right)}\right)_{*}\left(\mathcal{O}_{\widetilde{\mathcal{Z}}_{\left(s_{\alpha}, s_{\beta}, s_{\alpha}, s_{\beta}\right)}}\right)=\mathcal{O}_{S_{\{\alpha, \beta\}}},
$$

and similarly with $\alpha$ and $\beta$ interchanged. This finishes the proof of the finite braid relation in type $\mathbf{B}_{2}$, hence also of the assertions of Theorem 1.4 .1 concerning the action of $B_{\text {aff }}^{\prime}$ on $\mathcal{D}^{b} \operatorname{Coh}(\widetilde{\mathfrak{g}})$.

\section{Restriction to $\tilde{\mathcal{N}}$}

Now we will derive the assertions of Theorem 1.4.1 concerning the action of $B_{\text {aff }}^{\prime}$ on $\mathcal{D}^{b} \operatorname{Coh}(\widetilde{\mathcal{N}})$. We keep the notations and assumptions as before.

Let $i: \widetilde{\mathcal{N}} \hookrightarrow \widetilde{\mathfrak{g}}$ denote the closed embedding. For $\alpha \in \Phi$, we recall that $S_{\alpha}^{\prime}:=$ $S_{\alpha} \cap(\widetilde{\mathcal{N}} \times \tilde{\mathcal{N}})$, and that $\Gamma_{i}$ denotes the graph of $i$, a closed subvariety of $\widetilde{\mathcal{N}} \times \widetilde{\mathfrak{g}}$. First, relations $(2)$ to $(4)$ of Theorem 1.1 .3 for the action on $\mathcal{D}^{b} \operatorname{Coh}(\tilde{\mathcal{N}})$ can be proved exactly as for the action on $\mathcal{D}^{b} \operatorname{Coh}(\widetilde{\mathfrak{g}})$ (see 1.6). Now we prove relations (1).

Lemma 4.1. The tensor product $\mathcal{O}_{\widetilde{\mathcal{N}} \times \tilde{\mathfrak{g}}} \stackrel{\stackrel{L}{\otimes} \tilde{\mathfrak{g}} \times \mathfrak{\mathfrak { g }}}{\mathcal{O}_{S_{\alpha}}}$ is concentrated in degree 0 , and is isomorphic to $(i \times i)_{*} \mathcal{O}_{S_{\alpha}^{\prime}}$.

Proof. As in the proof of Proposition 1.5.2, we only have to consider the situation over $(B / B) \times\left(U^{+} B / B\right) \cong U^{+}$. We use the isomorphism $U^{+} \cong U_{(\alpha)}^{+} \times U_{\alpha}$, and choose coordinates $u$ on $U_{(\alpha)}^{+}, t$ on $U_{\alpha}$. On the fiber we use coordinates $e_{\gamma}^{(j)}, h_{\delta}^{(j)}$ $(j=1,2)$.

Then $\left.(\tilde{\mathcal{N}} \times \widetilde{\mathfrak{g}})\right|_{(B / B) \times\left(U^{+} B / B\right)}$ is defined by the equations $h_{\delta}^{(1)}=0(\delta \in \Phi)$, and $S_{\alpha}$ by $e_{\gamma}^{(1)}=e_{\gamma}^{(2)}, h_{\delta}^{(1)}=h_{\delta}^{(2)}, u=1$ and $h_{\alpha}^{(1)}-t e_{\alpha}^{(1)}$. The union of these equations forms a regular sequence, which proves the result.

Remark 4.2. These computations show that $S_{\alpha}^{\prime}$ is reduced. It is not irreducible (see 6.1 for details). 
Corollary 4.3. There exist isomorphisms in $\mathcal{D}_{\text {prop }}^{b} \operatorname{Coh}(\widetilde{\mathcal{N}} \times \widetilde{\mathfrak{g}})$ :

$$
\begin{aligned}
\mathcal{O}_{\Gamma_{i}} * \mathcal{O}_{S_{\alpha}^{\prime}} & \cong \mathcal{O}_{S_{\alpha}} * \mathcal{O}_{\Gamma_{i}}, \\
\mathcal{O}_{\Gamma_{i}} * \mathcal{O}_{S_{\alpha}^{\prime}}(\rho-\alpha,-\rho) & \cong \mathcal{O}_{S_{\alpha}}(\rho-\alpha,-\rho) * \mathcal{O}_{\Gamma_{i}} .
\end{aligned}
$$

Proof. We only prove the first isomorphism; the second one can be obtained similarly. It follows from Lemma 1.2 .3 that $\mathcal{O}_{\Gamma_{i}} * \mathcal{O}_{S_{\alpha}^{\prime}} \cong\left(\operatorname{Id}_{\widetilde{\mathcal{N}}} \times i\right)_{*} \mathcal{O}_{S_{\alpha}^{\prime}}$. Hence we only have to prove that $\mathcal{O}_{S_{\alpha}} * \mathcal{O}_{\Gamma_{i}} \cong\left(\operatorname{Id}_{\widetilde{\mathcal{N}}} \times i\right)_{*} \mathcal{O}_{S_{\alpha}^{\prime}}$.

Let $p_{a, b}$ denote the projection from $\widetilde{\mathcal{N}} \times \widetilde{\mathfrak{g}} \times \widetilde{\mathfrak{g}}$ to $\widetilde{\mathcal{N}} \times \widetilde{\mathfrak{g}}$ or $\widetilde{\mathfrak{g}} \times \widetilde{\mathfrak{g}}$, and $\Delta$ : $\widetilde{\mathcal{N}} \rightarrow \widetilde{\mathcal{N}} \times \widetilde{\mathcal{N}}$ denote the diagonal embedding. Then by definition $\mathcal{O}_{S_{\alpha}} * \mathcal{O}_{\Gamma_{i}}=$ $R\left(p_{1,3}\right)_{*}\left(p_{1,2}^{*} \mathcal{O}_{\Gamma_{i}} \stackrel{L}{\otimes} p_{2,3}^{*} \mathcal{O}_{S_{\alpha}}\right)$. But $p_{1,2}^{*} \mathcal{O}_{\Gamma_{i}} \cong\left(\operatorname{Id}_{\widetilde{\mathcal{N}}} \times i \times \operatorname{Id}_{\tilde{\mathfrak{g}}}\right)_{*}\left(\Delta \times \operatorname{Id}_{\tilde{\mathfrak{g}}}\right)_{*} \mathcal{O}_{\widetilde{\mathcal{N}} \times \tilde{\mathfrak{g}}}$. The result follows, using the projection formula and the preceding lemma, which implies that $L\left(i \times \operatorname{Id}_{\tilde{\mathfrak{g}}}\right)^{*} \mathcal{O}_{S_{\alpha}} \cong\left(\operatorname{Id}_{\widetilde{\mathcal{N}}} \times i\right)_{*} \mathcal{O}_{S_{\alpha}^{\prime}}$.

Corollary 4.4. The finite braid relations (i.e. relations (1) of Theorem 1.1.3) are satisfied by the kernels $\mathcal{O}_{S_{\alpha}^{\prime}}(\alpha \in \Phi)$.

Proof. First, let us prove an analogue of Proposition 1.5 .2 for the kernels $\mathcal{O}_{S_{\alpha}^{\prime}}$, i.e., that we have

$$
\mathcal{O}_{S_{\alpha}^{\prime}} *\left(\mathcal{O}_{S_{\alpha}^{\prime}}(\rho-\alpha,-\rho)\right) \cong \Delta_{*} \mathcal{O}_{\widetilde{\mathcal{N}}} \cong\left(\mathcal{O}_{S_{\alpha}^{\prime}}(\rho-\alpha,-\rho)\right) * \mathcal{O}_{S_{\alpha}^{\prime}} .
$$

Multiplying the equality $\mathcal{O}_{S_{\alpha}} *\left(\mathcal{O}_{S_{\alpha}}(\rho-\alpha,-\rho)\right)=\Delta_{*} \mathcal{O}_{\mathfrak{g}}$ with $\mathcal{O}_{\Gamma_{i}}$ on the right, and using Lemma 1.2 .3 and Corollary 4.3 , one obtains

$$
\left(\operatorname{Id}_{\widetilde{\mathcal{N}}} \times i\right)_{*}\left(\mathcal{O}_{S_{\alpha}^{\prime}} *\left(\mathcal{O}_{S_{\alpha}^{\prime}}(\rho-\alpha,-\rho)\right)\right) \cong\left(\operatorname{Id}_{\widetilde{\mathcal{N}}} \times i\right)_{*}\left(\Delta_{*} \mathcal{O}_{\widetilde{\mathcal{N}}}\right)
$$

It follows that the complex of sheaves $\mathcal{O}_{S_{\alpha}^{\prime}} *\left(\mathcal{O}_{S_{\alpha}^{\prime}}(\rho-\alpha,-\rho)\right)$ has its cohomology concentrated in degree 0 , i.e., is isomorphic to a coherent sheaf on $\widetilde{\mathcal{N}} \times \widetilde{\mathcal{N}}$. Then, as $\left(\operatorname{Id}_{\widetilde{\mathcal{N}}} \times i\right)_{*}: \operatorname{Coh}(\widetilde{\mathcal{N}} \times \widetilde{\mathcal{N}}) \rightarrow \operatorname{Coh}(\widetilde{\mathcal{N}} \times \widetilde{\mathfrak{g}})$ has a left inverse $\left(\operatorname{Id}_{\widetilde{\mathcal{N}}} \times i\right)^{*}$, we deduce the first isomorphism in $(\dagger)$. The second one can be proved similarly.

Now, let us prove that the braid relations are satisfied. To fix notation, assume that $\alpha$ and $\beta$ are simple roots generating a root system of type $\mathbf{A}_{2}$ (the other cases can be treated similarly). We have to prove that $\mathcal{O}_{S_{\alpha}^{\prime}} * \mathcal{O}_{S_{\beta}^{\prime}} * \mathcal{O}_{S_{\alpha}^{\prime}} \cong \mathcal{O}_{S_{\beta}^{\prime}} * \mathcal{O}_{S_{\alpha}^{\prime}} * \mathcal{O}_{S_{\beta}^{\prime}}$. By $(\dagger)$, this is equivalent to

$$
\mathcal{O}_{S_{\beta}^{\prime}}(\rho-\beta,-\rho) * \mathcal{O}_{S_{\alpha}^{\prime}}(\rho-\alpha,-\rho) * \mathcal{O}_{S_{\beta}^{\prime}}(\rho-\beta,-\rho) * \mathcal{O}_{S_{\alpha}^{\prime}} * \mathcal{O}_{S_{\beta}^{\prime}} * \mathcal{O}_{S_{\alpha}^{\prime}} \cong \Delta_{*} \mathcal{O}_{\widetilde{\mathcal{N}}} .
$$

But we know (see section 2) that

$$
\mathcal{O}_{S_{\beta}}(\rho-\beta,-\rho) * \mathcal{O}_{S_{\alpha}}(\rho-\alpha,-\rho) * \mathcal{O}_{S_{\beta}}(\rho-\beta,-\rho) * \mathcal{O}_{S_{\alpha}} * \mathcal{O}_{S_{\beta}} * \mathcal{O}_{S_{\alpha}} \cong \Delta_{*} \mathcal{O}_{\mathfrak{\mathfrak { g }}}
$$

Hence we can use the same argument as in the first part of this proof.

Remark 4.5. The restriction of this action to $B_{\text {aff }}$, for $R$ of type $\mathbf{A}$, was also considered in $[\mathrm{KT}$. There, it was proved to have some nice properties.

\section{RELATION TO LOCALIZATION IN POSITIVE CHARACTERISTIC}

In this section we show that the action of $B_{\text {aff }}^{\prime}$ on $\mathcal{D}^{b} \operatorname{Coh}(\widetilde{\mathfrak{g}})$ we have constructed above, or rather the similar action on $\mathcal{D}^{b} \operatorname{Coh}\left(\widetilde{\mathfrak{g}}^{(1)}\right)$ (for $\widetilde{\mathfrak{g}}^{(1)}$ the Frobenius twist of $\widetilde{\mathfrak{g}}$, see [BMR, 1.1.1]), extends the action on $\mathcal{D}^{b} \operatorname{Coh}_{\mathcal{B}_{\chi}^{(1)}}\left(\widetilde{\mathfrak{g}}^{(1)}\right)$ constructed in BMR2] using representation theory of Lie algebras and $\mathcal{D}$-modules in positive characteristic (see below, or [BMR], for the notation). 
In 5.1 and 5.4 we assume $\operatorname{char}(\mathbb{k})>h$ for $h$ the Coxeter number of $G$. In 5.2 and 5.3. $\mathbb{k}$ is an arbitrary algebraically closed field. We use the same notation as above. In particular, $G$ has no component of type $\mathbf{G} 26$.

If $X$ is a scheme and $Y \subset X$ is a closed subscheme, one says that a quasi-coherent sheaf $\mathcal{F}$ on $X$ is supported on $Y$ if $\mathcal{F}_{x}=0$ for $x \notin Y$ (see [BMR, 3.1.7]). If $\mathcal{F}$ is coherent, and if $\mathcal{I}_{Y} \subset \mathcal{O}_{X}$ is the ideal defining $Y$, this is equivalent to requiring that the action of $\mathcal{I}_{Y}$ on $\mathcal{F}$ be locally nilpotent. We let $\operatorname{Coh}_{Y}(X)$ denote the subcategory of $\operatorname{Coh}(X)$ whose objects are coherent sheaves supported on $Y$.

5.1. Review of the results of $\mathrm{BMR}$ and $\mathrm{BMR2}$. In this paragraph we recall some results of [BMR] and BMR2] that relate representation theory of Lie algebras with coherent sheaves on $\tilde{\mathcal{N}}$ and $\widetilde{\mathfrak{g}}$ (or parabolic analogs).

Let $\mathfrak{Z}$ be the center of $\mathcal{U} \mathfrak{g}$, the enveloping algebra of $\mathfrak{g}$. The subalgebra of $G$ invariants, $\mathfrak{Z}_{\mathrm{HC}}:=(\mathcal{U} \mathfrak{g})^{G}$ is central in $\mathcal{U} \mathfrak{g}$. This is the "Harish-Chandra part" of $\mathfrak{Z}$, which is isomorphic to $S(\mathfrak{t})^{(W, \bullet)}$, the algebra of $W$-invariants in the symmetric algebra of $\mathfrak{t}$, for the dot-action. This is an analog of the center of the enveloping algebra in characteristic 0 . The center $\mathfrak{Z}$ also has another part, the "Frobenius part" $\mathfrak{Z}_{\mathrm{Fr}}$ which is generated, as an algebra, by the elements $X^{p}-X^{[p]}$ for $X \in \mathfrak{g}$. It is isomorphic to $S\left(\mathfrak{g}^{(1)}\right)$, the functions on the Frobenius twist of $\mathfrak{g}^{*}$. Under our assumption $p>h$, there is an isomorphism (see e.g. $\mathrm{MR}$ ):

$$
\mathfrak{Z}_{\mathrm{HC}} \otimes_{\mathfrak{Z}_{\mathrm{Fr}} \cap \mathfrak{Z}_{\mathrm{HC}}} \mathfrak{\mathfrak { Z } F r} \stackrel{\sim}{\rightarrow} \mathfrak{Z} .
$$

Hence, a character of $\mathfrak{Z}$ is given by a compatible pair $(\lambda, \chi) \in \mathfrak{t}^{*} \times \mathfrak{g}^{*(1)}$. Here we will only consider the case when $\chi$ is nilpotent, and $\lambda \in \mathfrak{t}^{*}$ is integral, i.e., in the image of the natural map $\mathbb{X} \rightarrow \mathfrak{t}^{*}$. If $\lambda \in \mathbb{X}$, we still denote by $\lambda$ its image in $\mathfrak{t}^{*}$. We denote the corresponding specializations by

$$
(\mathcal{U} \mathfrak{g})^{\lambda}:=(\mathcal{U} \mathfrak{g}) \otimes_{\mathfrak{J}_{\mathrm{HC}}} \mathbb{k}_{\lambda},(\mathcal{U} \mathfrak{g})_{\chi}:=(\mathcal{U} \mathfrak{g}) \otimes_{\mathfrak{J}_{\mathrm{Fr}}} \mathbb{k}_{\chi},(\mathcal{U} \mathfrak{g})_{\chi}^{\lambda}:=(\mathcal{U} \mathfrak{g}) \otimes_{\mathfrak{Z}} \mathbb{k}_{(\lambda, \chi)}
$$

Recall the variety $\tilde{\mathfrak{g}}_{\alpha}$ defined in 1.4 . For $\chi \in \mathfrak{g}^{*}$ nilpotent we define $\mathcal{B}_{\chi}$, respectively $\mathcal{P}_{\alpha, \chi}$, as the inverse image of $\chi$ under $\widetilde{\mathfrak{g}} \rightarrow \mathfrak{g}^{*}$, respectively $\widetilde{\mathfrak{g}}_{\alpha} \rightarrow \mathfrak{g}^{*}$. The variety $\mathcal{B}_{\chi}$ is isomorphic to the Springer fiber associated to $\chi$.

Let $\operatorname{Mod}_{(\lambda, \chi)}^{\mathrm{fg}}(\mathcal{U} \mathfrak{g})$ denote the abelian category of finitely generated $\mathcal{U} \mathfrak{g}$-modules on which $\mathfrak{Z}$ acts with generalized character $(\lambda, \chi)$, and similarly for $\operatorname{Mod}_{\chi}^{\mathrm{fg}}\left((\mathcal{U} \mathfrak{g})^{\lambda}\right)$, $\operatorname{Mod}_{\lambda}^{\mathrm{fg}}\left((\mathcal{U} \mathfrak{g})_{\chi}\right), \operatorname{Mod}^{\mathrm{fg}}\left((\mathcal{U} \mathfrak{g})_{\chi}^{\lambda}\right)$. We have (see [BMR, 5.3.1] for (i), BMR2, 1.5.1.c, 1.5.2.b] for (ii)):

Theorem 5.1.1. (i) Let $\chi \in \mathfrak{g}^{*}$ be nilpotent, and $\lambda \in \mathbb{X}$ regular. There exist equivalences

$$
\begin{array}{ccc}
\gamma_{(\lambda, \chi)}^{\mathcal{B}}: & \mathcal{D}^{b} \operatorname{Coh}_{\mathcal{B}_{\chi}^{(1)}}\left(\widetilde{\mathfrak{g}}^{(1)}\right) & \stackrel{\sim}{\longrightarrow} \mathcal{D}^{b} \operatorname{Mod}_{(\lambda, \chi)}^{\mathrm{fg}}(\mathcal{U} \mathfrak{g}), \\
\gamma_{\chi}^{\mathcal{B}, \lambda}: & \mathcal{D}^{b} \operatorname{Coh}_{\mathcal{B}_{\chi}^{(1)}}\left(\widetilde{\mathcal{N}}^{(1)}\right) & \stackrel{\sim}{\longrightarrow} \mathcal{D}^{b} \operatorname{Mod}_{\chi}^{\mathrm{fg}}\left((\mathcal{U} \mathfrak{g})^{\lambda}\right) .
\end{array}
$$

(ii) Again, let $\chi \in \mathfrak{g}^{*}$ be nilpotent. Fix $\alpha \in \Phi$ and let $\mu \in \mathbb{X}$ be on the reflection hyperplane of $s_{\alpha}$ for the dot-action, but not on any other reflection hyperplane. There exists an equivalence

$$
\gamma_{(\lambda, \chi)}^{\mathcal{P}_{\alpha}}: \quad \mathcal{D}^{b} \operatorname{Coh}_{\mathcal{P}_{\alpha, \chi}^{(1)}}\left(\widetilde{\mathfrak{g}}_{\alpha}^{(1)}\right) \stackrel{\sim}{\longrightarrow} \mathcal{D}^{b} \operatorname{Mod}_{(\mu, \chi)}^{\mathrm{fg}}(\mathcal{U} \mathfrak{g}) .
$$

\footnotetext{
${ }^{6}$ This assumption is used only in 5.4
} 
Remark 5.1.2. (i) The categories of coherent sheaves we are using here are not exactly the same as the ones used in BMR. More precisely, we have taken $\mathrm{Coh}_{\mathcal{B}_{\chi}^{(1)}}\left(\widetilde{\mathfrak{g}}^{(1)}\right)$ instead of $\mathrm{Coh}_{\mathcal{B}_{\chi}^{(1)} \times\{\lambda\}}\left(\widetilde{\mathfrak{g}}^{(1)} \times_{\mathfrak{t}^{*(1)}} \mathfrak{t}^{*}\right)$. But, as remarked in BMR2, 1.5.3. (c)], the projection $\widetilde{\mathfrak{g}}^{(1)} \times \mathrm{t}^{*(1)} \mathfrak{t}^{*} \rightarrow \widetilde{\mathfrak{g}}^{(1)}$ induces an isomorphism between the formal neighborhoods of $\mathcal{B}_{\chi}^{(1)} \times\{\lambda\}$ and $\mathcal{B}_{\chi}^{(1)}$.

(ii) These equivalences depend on choices of splitting bundles. We follow the conventions of BMR2, 1.3.5], and denote by $\mathcal{M}_{(\lambda, \chi)}^{\mathcal{B}}$ the vector bundle on the formal neighborhood of $\mathcal{B}_{\chi}^{(1)}$ in $\widetilde{\mathfrak{g}}^{(1)}$ which intervenes in the definition of $\gamma_{(\lambda, \chi)}^{\mathcal{B}}$. Our notations for equivalences are consistent with [BMR2, 2.2.5].

The translation functors $T_{\lambda}^{\mu}: \operatorname{Mod}_{(\lambda, \chi)}^{\mathrm{fg}}(\mathcal{U} \mathfrak{g}) \rightarrow \operatorname{Mod}_{(\mu, \chi)}^{\mathrm{fg}}(\mathcal{U} \mathfrak{g})$ are defined in BMR, 6.1]. Recall the natural morphism $\tilde{\pi}_{\alpha}: \tilde{\mathfrak{g}} \rightarrow \tilde{\mathfrak{g}}_{\alpha}$ (see 1.4). Then (BMR2, $2.2 .5])$ :

Proposition 5.1.3. Let $\lambda, \mu, \alpha$ be as in Theorem 5.1.1. Assume that $\mu$ lies in the closure of the facet of $\lambda$. There exist isomorphisms of functors

$$
T_{\lambda}^{\mu} \circ \gamma_{(\lambda, \chi)}^{\mathcal{B}} \cong \gamma_{(\mu, \chi)}^{\mathcal{P}_{\alpha}} \circ R\left(\widetilde{\pi}_{\alpha}^{(1)}\right)_{*} \quad \text { and } \quad T_{\mu}^{\lambda} \circ \gamma_{(\mu, \chi)}^{\mathcal{P}_{\alpha}} \cong \gamma_{(\lambda, \chi)}^{\mathcal{B}} \circ L\left(\widetilde{\pi}_{\alpha}^{(1)}\right)^{*} .
$$

5.2. The reflection functors. Let us fix a simple root $\alpha \in \Phi$. In this paragraph we study the functor $L\left(\widetilde{\pi}_{\alpha}^{(1)}\right)^{*} \circ R\left(\widetilde{\pi}_{\alpha}^{(1)}\right)_{*}$. To simplify notation, we forget about the Frobenius twists; the "twisted versions" of our results can be proved similarly. In this paragraph and the next one, $\operatorname{char}(\mathbb{k})$ is arbitrary.

We are in the situation of Lemma 1.2 .2 , with $f$ being the morphism $\tilde{\pi}_{\alpha}$. So $L\left(\widetilde{\pi}_{\alpha}\right)^{*} \circ R\left(\widetilde{\pi}_{\alpha}\right)_{*}$ is the convolution functor with kernel

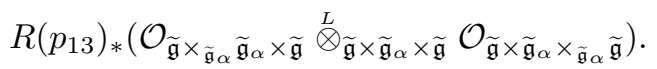

The situation is particularly simple here, due to the following result:

Lemma 5.2.1. The derived tensor product $\mathcal{O}_{\tilde{\mathfrak{g}} \times{ }_{\tilde{\mathfrak{g}} \alpha} \tilde{\mathfrak{g}}_{\alpha} \times \tilde{\mathfrak{g}}} \stackrel{L}{\otimes_{\tilde{\mathfrak{g}}} \times \widetilde{\mathfrak{g}}_{\alpha} \times \tilde{\mathfrak{g}}} \mathcal{O}_{\tilde{\mathfrak{g}} \times \widetilde{\mathfrak{g}}_{\alpha} \times{ }_{\tilde{\mathfrak{g}} \alpha} \tilde{\mathfrak{g}}}$ is concentrated in degree 0 . It equals the sheaf of functions on the intersection $\left(\tilde{\mathfrak{g}} \times_{\tilde{\mathfrak{g}}_{\alpha}}\right.$ $\left.\widetilde{\mathfrak{g}}_{\alpha} \times \widetilde{\mathfrak{g}}\right) \cap\left(\widetilde{\mathfrak{g}} \times \widetilde{\mathfrak{g}}_{\alpha} \times_{\tilde{\mathfrak{g}}_{\alpha}} \widetilde{\mathfrak{g}}\right)$. Moreover, this intersection is reduced.

Proof. This proof is again similar to the proof of Proposition 1.5.2. For simplicity, in this proof we write $P$ for $P_{\alpha}$. We can restrict to the situation over $(B / B) \times$ $\left(U^{+} P / P\right) \times\left(U^{+} B / B\right) \cong U_{(\alpha)}^{+} \times U^{+}$. We use the isomorphisms $\left.\widetilde{\mathfrak{g}}\right|_{U^{+} B / B} \cong\left(\mathfrak{b}^{+}\right)^{*} \times$ $U^{+}$and $\left.\widetilde{\mathfrak{g}}_{\alpha}\right|_{U^{+} P / P} \cong\left(\mathfrak{b}^{+} \oplus \mathbb{k} e_{-\alpha}\right)^{*} \times U_{(\alpha)}^{+}$induced by restriction, and choose as usual coordinates $e_{\gamma}^{(i)}, h_{\delta}^{(i)}\left(\gamma \in R^{+}, \delta \in \Phi, i \in\{1,2,3\}\right)$ and $e_{-\alpha}^{(2)}$ in the fibers, $u^{(2)}$ and $u^{(3)}$ on $U_{(\alpha)}^{+}$, and $t$ on $U_{\alpha}$.

The equations of the first subvariety are $e_{\gamma}^{(1)}=e_{\gamma}^{(2)}, h_{\delta}^{(1)}=h_{\delta}^{(2)}, e_{-\alpha}^{(2)}=0$ and $u^{(2)}=1$. And the equations of the second variety are $e_{\gamma}^{(2)}=e_{\gamma}^{(3)}, h_{\delta}^{(2)}=h_{\delta}^{(3)}$, $u^{(2)}=u^{(3)}$ and $u^{(2)} \cdot u_{\alpha}(t) \cdot e_{-\alpha}^{(2)}=0$, i.e., $u^{(2)} \cdot\left(e_{-\alpha}^{(2)}+t h_{\alpha}^{(2)}-t^{2} e_{\alpha}^{(2)}\right)=0$.

It is clear that these equations form a regular sequence, and define a reduced scheme. This proves the lemma.

The morphism $p_{1,3}$ restricts to an isomorphism from the intersection $\left(\widetilde{\mathfrak{g}} \times_{\tilde{\mathfrak{g}}_{\alpha}} \widetilde{\mathfrak{g}}_{\alpha} \times\right.$ $\widetilde{\mathfrak{g}}) \cap\left(\widetilde{\mathfrak{g}} \times \widetilde{\mathfrak{g}}_{\alpha} \times_{\widetilde{\mathfrak{g}}_{\alpha}} \widetilde{\mathfrak{g}}\right)$ to $\widetilde{\mathfrak{g}} \times_{\widetilde{\mathfrak{g}}_{\alpha}} \widetilde{\mathfrak{g}}$. Hence we obtain, using Lemma 1.2.2. 
Proposition 5.2.2. There exists an isomorphism of functors

$$
L\left(\widetilde{\pi}_{\alpha}\right)^{*} \circ R\left(\widetilde{\pi}_{\alpha}\right)_{*} \cong F_{\widetilde{\mathfrak{g}} \rightarrow \tilde{\mathfrak{g}}}^{\mathcal{O}_{\tilde{\mathfrak{g}}} \times \tilde{\mathfrak{g}}_{\alpha} \tilde{\mathfrak{g}}}
$$

for the closed subvariety $\widetilde{\mathfrak{g}} \times_{\widetilde{\mathfrak{g}}_{\alpha}} \widetilde{\mathfrak{g}} \subset \widetilde{\mathfrak{g}} \times \tilde{\mathfrak{g}}$.

Moreover, under this isomorphism, the adjunction morphism $L\left(\widetilde{\pi}_{\alpha}\right)^{*} \circ R\left(\widetilde{\pi}_{\alpha}\right)_{*} \rightarrow$ Id is induced by the restriction map $\mathcal{O}_{\tilde{\mathfrak{g}} \times{ }_{\mathfrak{\mathfrak { g }}} \tilde{\mathfrak{g}}} \rightarrow \Delta_{*} \mathcal{O}_{\tilde{\mathfrak{g}}}$.

5.3. Intertwining functors. We have seen in 1.4 that $\widetilde{\mathfrak{g}} \times_{\widetilde{\mathfrak{g}}_{\alpha}} \widetilde{\mathfrak{g}}=G \times{ }^{B} \mathscr{R}_{\alpha}$, and that the $B$-variety $\mathscr{R}_{\alpha}$ has two irreducible components, $\mathscr{D}_{\alpha}$ and $\mathscr{S}_{\alpha}$.

Lemma 5.3.1. There exist exact sequences of B-equivariant quasi-coherent sheaves on $\mathfrak{g}^{*} \times P_{\alpha} / B$, where the surjections are restriction maps:

$$
\begin{gathered}
\mathcal{O}_{\mathscr{D}_{\alpha}} \hookrightarrow \mathcal{O}_{\mathscr{R}_{\alpha}} \rightarrow \mathcal{O}_{\mathscr{S}_{\alpha}}, \\
\mathcal{O}_{\mathscr{S}_{\alpha}}(-\rho) \otimes_{\mathbb{k}} \mathbb{k}_{B}(\rho-\alpha) \hookrightarrow \mathcal{O}_{\mathscr{R}_{\alpha}} \rightarrow \mathcal{O}_{\mathscr{D}_{\alpha}} .
\end{gathered}
$$

Proof. We use the same notations as in 1.4. In particular, recall the equations of $\mathscr{R}_{\alpha}, \mathscr{S}_{\alpha}, \mathscr{D}_{\alpha}$. On $U_{\alpha} B / B$, we have an exact sequence

$$
\mathbb{k}\left[h_{\delta}, e_{\gamma}, t\right] /(t) \hookrightarrow \mathbb{k}\left[h_{\delta}, e_{\gamma}, t\right] /\left(t\left(h_{\alpha}-t e_{\alpha}\right)\right) \rightarrow \mathbb{k}\left[h_{\delta}, e_{\gamma}, t\right] /\left(h_{\alpha}-t e_{\alpha}\right)
$$

where the first map is multiplication by $\left(h_{\alpha}-t e_{\alpha}\right)$. Under the change of coordinates on $\left(U_{\alpha} B / B\right) \cap\left(n_{\alpha} U_{\alpha} B / B\right)$ (given by $\left.t \mapsto-\frac{1}{t}\right), h_{\alpha}-t e_{\alpha}$ is sent to $h_{\alpha}+\frac{1}{t} e_{\alpha}$, which is 0 in $\mathbb{k}\left[\left.\mathscr{R}_{\alpha}\right|_{\left(n_{\alpha} U_{\alpha} B / B\right)-\left\{n_{\alpha} B / B\right\}} \cong \mathbb{k}\left[h_{\delta}, e_{\gamma}, t^{ \pm 1}\right] /\left(e_{\alpha}+t h_{\alpha}\right)\right.$. Hence we can glue the preceding exact sequence with the trivial exact sequence $0 \hookrightarrow \mathbb{k}\left[h_{\delta}, e_{\gamma}, t\right] /\left(e_{\alpha}+\right.$ $\left.t h_{\alpha}\right) \rightarrow \mathbb{k}\left[h_{\delta}, e_{\gamma}, t\right] /\left(e_{\alpha}+t h_{\alpha}\right)$ to obtain an exact sequence of sheaves

$$
\mathcal{O}_{\mathscr{D}_{\alpha}} \hookrightarrow \mathcal{O}_{\mathscr{R}_{\alpha}} \rightarrow \mathcal{O}_{\mathscr{S}_{\alpha}} .
$$

This sequence is obviously $B$-equivariant (the first map is non zero only over $B / B$, and $h_{\alpha}$ is $B$-invariant in our coordinate ring). This gives the first exact sequence of the lemma.

Similarly, we have an exact sequence

$$
\mathbb{k}\left[h_{\delta}, e_{\gamma}, t\right] /\left(h_{\alpha}-t e_{\alpha}\right) \hookrightarrow \mathbb{k}\left[h_{\delta}, e_{\gamma}, t\right] /\left(t\left(h_{\alpha}-t e_{\alpha}\right)\right) \rightarrow \mathbb{k}\left[h_{\delta}, e_{\gamma}, t\right] /(t)
$$

where the first map is multiplication by $t$. To glue this exact sequence with the trivial one on $n_{\alpha} U_{\alpha} B / B$ :

$$
\mathbb{k}\left[h_{\delta}, e_{\gamma}, t\right] /\left(e_{\alpha}+t h_{\alpha}\right) \hookrightarrow \mathbb{k}\left[h_{\delta}, e_{\gamma}, t\right] /\left(e_{\alpha}+t h_{\alpha}\right) \rightarrow 0
$$

we have to tensor $\mathcal{O}_{\mathscr{S}_{\alpha}}$ with the inverse image of $\mathcal{O}_{P_{\alpha} / B}(-\rho) \cong \mathcal{O}_{\mathbb{P}^{1}}(-1)$ on $P_{\alpha} / B \cong$ $\mathbb{P}_{\mathbb{k}}^{1}$. We obtain the exact sequence of quasi-coherent sheaves

$$
\mathcal{O}_{\mathscr{S}_{\alpha}}(-\rho) \hookrightarrow \mathcal{O}_{\mathscr{R}_{\alpha}} \rightarrow \mathcal{O}_{\mathscr{D}_{\alpha}} .
$$

To understand the $B$-equivariant structure of the first morphism, we observe that to define a morphism $\mathcal{O}_{P_{\alpha} / B}(-\rho) \rightarrow \mathcal{O}_{P_{\alpha} / B}$ is equivalent to choosing a vector in $\Gamma\left(P_{\alpha} / B, \mathcal{O}_{P_{\alpha} / B}(\rho)\right)$. This $P_{\alpha}$-module has dimension two, with weights $\rho$ and $\rho-\alpha$. The line of weight $\rho-\alpha$ is $B$-stable: choosing a non-zero vector in this line thus defines a morphism of $B$-equivariant sheaves

$$
\mathcal{O}_{\mathscr{S}_{\alpha}}(-\rho) \otimes_{\mathbb{k}} \mathbb{k}_{B}(\rho-\alpha) \rightarrow \mathcal{O}_{\mathscr{R}_{\alpha}},
$$

which yields the second exact sequence of the lemma.

Inducing these exact sequences from $B$ to $G$, we obtain 
Corollary 5.3.2. There exist exact sequences of quasi-coherent sheaves on $\mathfrak{g}^{*} \times$ $\left(\mathcal{B} \times \mathcal{P}_{\alpha} \mathcal{B}\right)$, where the surjections are restriction maps:

$$
\begin{gathered}
\mathcal{O}_{\Delta \tilde{\mathfrak{g}}} \hookrightarrow \mathcal{O}_{\tilde{\mathfrak{g}} \times \tilde{\mathfrak{g}}_{\alpha} \tilde{\mathfrak{g}}} \rightarrow \mathcal{O}_{S_{\alpha}}, \\
\mathcal{O}_{S_{\alpha}}(\rho-\alpha,-\rho) \hookrightarrow \mathcal{O}_{\tilde{\mathfrak{g}} \times \tilde{\mathfrak{g}}_{\alpha} \tilde{\mathfrak{g}}} \rightarrow \mathcal{O}_{\Delta \tilde{\mathfrak{g}}} .
\end{gathered}
$$

Remark 5.3.3. As in Proposition 1.5.2, $\rho$ can be replaced by any $\lambda \in \mathbb{X}$ with $\left\langle\lambda, \alpha^{\vee}\right\rangle=1$.

5.4. The two actions of the braid group coincide. Assume again that $p=$ $\operatorname{char}(\mathbb{k})>h$. Let us fix some $\lambda \in \mathbb{X}$ in the alcove $\mathscr{C}_{0}=\left\{\nu \in \mathbb{X} \otimes \mathbb{R} \mid \forall \beta \in R^{+}, 0<\right.$ $\left.\left\langle\nu+\rho, \beta^{\vee}\right\rangle<p\right\}$, and some $\chi \in \mathfrak{g}^{*}$ nilpotent. In this paragraph we finally prove that the "Frobenius twisted version" of the action of $B_{\text {aff }}^{\prime}$ on $\mathcal{D}^{b} \mathrm{Coh}(\widetilde{\mathfrak{g}})$ considered in Theorem 1.4.1 extends the action of $B_{\text {aff }}^{\prime}$ on $\mathcal{D}^{b} \operatorname{Coh}_{\mathcal{B}_{\chi}^{(1)}}\left(\widetilde{\mathfrak{g}}^{(1)}\right)$ coming from BMR2, 2.1.6, 2.3.2], via $\gamma_{(\lambda, \chi)}^{\mathcal{B}}$. More precisely, for $b \in B_{\text {aff }}^{\prime}$ we denote by

$$
\begin{gathered}
\mathbf{J}^{b}: \mathcal{D}^{b} \operatorname{Coh}\left(\widetilde{\mathfrak{g}}^{(1)}\right) \rightarrow \mathcal{D}^{b} \operatorname{Coh}\left(\widetilde{\mathfrak{g}}^{(1)}\right), \quad \text { respectively } \\
\mathbf{I}_{(\lambda, \chi)}^{b}: \mathcal{D}^{b} \operatorname{Mod}_{(\lambda, \chi)}^{\mathrm{fg}}(\mathcal{U} \mathfrak{g}) \rightarrow \mathcal{D}^{b} \operatorname{Mod}_{(\lambda, \chi)}^{\mathrm{fg}}(\mathcal{U} \mathfrak{g})
\end{gathered}
$$

the action of $b$ coming from Theorem 1.4.1 respectively the action constructed in BMR2, 2.1.4]7. The functor $\mathbf{J}^{b}$ restricts to an auto-equivalence of $\mathcal{D}^{b} \operatorname{Coh}_{\mathcal{B}_{\chi}^{(1)}}\left(\widetilde{\mathfrak{g}}^{(1)}\right)$, denoted similarly. The main result of this paragraph is the following:

Theorem 5.4.1. For any $b \in B_{\mathrm{aff}}^{\prime}$ there exists an isomorphism of functors from $\mathcal{D}^{b} \operatorname{Coh}_{\mathcal{B}_{\chi}^{(1)}}\left(\widetilde{\mathfrak{g}}^{(1)}\right)$ to itself:

$$
\mathbf{J}^{b} \cong\left(\gamma_{(\lambda, \chi)}^{\mathcal{B}}\right)^{-1} \circ \mathbf{I}_{(\lambda, \chi)}^{b} \circ \gamma_{(\lambda, \chi)}^{\mathcal{B}} .
$$

Proof. It is enough to consider the generators $T_{\alpha}$ (denoted by $\widetilde{s_{\alpha}}$ in BMR2]) and $\theta_{x}$, for $\alpha \in \Phi$ and $x \in \mathbb{X}$. First, fix some $x \in \mathbb{X}$. It is proven in [BMR2, 2.3.3] that $\theta_{x}$ for $x \in \mathbb{X}$ dominant acts (in the action of [BMR2]) by convolution with kernel $\Delta_{*} \mathcal{O}_{\widetilde{\mathfrak{g}}^{(1)}}(x)$. It follows, by construction, that this result is true for any $x \in \mathbb{X}$. Hence the two actions coincide for $b=\theta_{x}$.

The case of $T_{\alpha}$ is more delicate, and will occupy the rest of the proof. We fix $\alpha \in \Phi$. We will construct an isomorphism of functors

$$
\left(\mathbf{I}_{(\lambda, \chi)}^{\alpha}\right)^{-1} \circ \gamma_{(\lambda, \chi)}^{\mathcal{B}} \cong \gamma_{(\lambda, \chi)}^{\mathcal{B}} \circ F_{\widetilde{\mathfrak{g}}_{(1) \rightarrow \mathfrak{g}^{(1)}}^{(1)}}^{\mathcal{O}}
$$

This is equivalent to the theorem for $b=T_{\alpha}$, due to Proposition 1.5.2, Let us choose some $\mu_{\alpha} \in \mathbb{X}$, on the $\alpha$-wall of $\mathscr{C}_{0}$ (and on no other wall). We define the functor $R_{\alpha}:=T_{\mu_{\alpha}}^{\lambda} \circ T_{\lambda}^{\mu_{\alpha}}$ (see [BMR2, 2.2.7]).

First, let us consider a single object $\mathcal{F} \in \mathcal{D}^{b} \operatorname{Coh}_{\mathcal{B}^{(1)}}\left(\widetilde{\mathfrak{g}}^{(1)}\right)$. Now we prove that the images of $\mathcal{F}$ under the two functors in (5.4.2) are isomorphic. Later we will prove that this isomorphism comes from an isomorphism of functors.

Lemma 5.4.3. There exists an isomorphism in $\mathcal{D}^{b} \operatorname{Coh}_{\mathcal{B}_{\chi}^{(1)}}\left(\widetilde{\mathfrak{g}}^{(1)}\right)$ :

$$
\left(\mathbf{I}_{(\lambda, \chi)}^{\alpha}\right)^{-1} \circ \gamma_{(\lambda, \chi)}^{\mathcal{B}}(\mathcal{F}) \cong \gamma_{(\lambda, \chi)}^{\mathcal{B}} \circ F_{\widetilde{\mathfrak{g}}^{(1)} \rightarrow \widetilde{\mathfrak{g}}^{(1)}}^{\mathcal{O}(1)}(\mathcal{F})
$$

\footnotetext{
${ }^{7}$ This action depends on the choice of an isomorphism between the "local" extended affine braid group and $B_{\text {aff }}^{\prime}$. We take the isomorphism associated to the choice of the element $\lambda \in W_{\text {aff }}^{\prime} \bullet \lambda$, as in BMR2 2.1.6].
} 
Proof of Lemma 5.4.3. By definition (see [BMR2, 2.2.4, 2.3.1]), there is an exact triangle

$$
\left(\mathbf{I}_{(\lambda, \chi)}^{\alpha}\right)^{-1} \circ \gamma_{(\lambda, \chi)}^{\mathcal{B}}(\mathcal{F}) \rightarrow R_{\alpha} \circ \gamma_{(\lambda, \chi)}^{\mathcal{B}}(\mathcal{F}) \rightarrow \gamma_{(\lambda, \chi)}^{\mathcal{B}}(\mathcal{F}),
$$

where the second arrow is induced by adjunction. By Propositions 5.1.3 and 5.2.2.

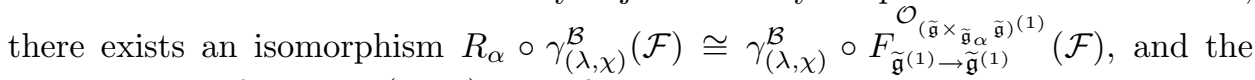
second arrow of triangle (5.4.4) identifies with the morphism

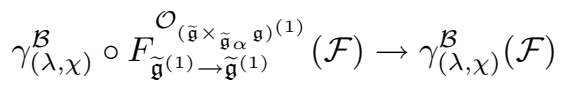

induced by the restriction map $\mathcal{O}_{\left(\widetilde{\mathfrak{g}} \times_{\left.\tilde{\mathfrak{g}}_{\alpha} \mathfrak{g}\right)}\right)^{(1)}} \rightarrow \mathcal{O}_{\Delta \widetilde{\mathfrak{g}}^{(1)}}$ (recall that the convolution with kernel $\mathcal{O}_{\Delta \tilde{\mathfrak{g}}^{(1)}}$ is the identity). Now the result follows from the second exact sequence in Corollary 5.3.2, using basic properties of triangulated categories.

Let $q_{1}, q_{2}: S_{\alpha}^{(1)} \rightarrow \widetilde{\mathfrak{g}}^{(1)}$ be the natural morphisms, induced by the projections $p_{1}, p_{2}: \widetilde{\mathfrak{g}}^{(1)} \times \widetilde{\mathfrak{g}}^{(1)} \rightarrow \widetilde{\mathfrak{g}}^{(1)}$. Then, $F_{\widetilde{\mathfrak{g}}_{S_{\alpha}^{(1)} \rightarrow \widetilde{\mathfrak{g}}^{(1)}}^{\mathcal{O}_{(1)}(-\rho, \rho-\alpha)}}$ is isomorphic to the functor

$$
\mathcal{F} \mapsto R\left(q_{2}\right)_{*}\left(L\left(q_{1}\right)^{*} \mathcal{F} \otimes_{S_{\alpha}^{(1)}} \mathcal{O}_{S_{\alpha}^{(1)}}(-\rho, \rho-\alpha)\right)
$$

(by the projection formula). We denote by $\mathcal{X}$ the completion of $\widetilde{\mathfrak{g}}^{(1)}$ along the closed subscheme $\mathcal{B}_{\chi}^{(1)}$, and by $\mathcal{Y}$ the completion of $S_{\alpha}^{(1)}$ along the closed subscheme $\mathcal{B}_{\chi}^{(1)} \times_{\mathcal{P}_{\alpha, \chi}^{(1)}} \mathcal{B}_{\chi}^{(1)}$. Then $q_{1}$ and $q_{2}$ induce morphisms of formal schemes $\widehat{q_{1}}, \widehat{q_{2}}: \mathcal{Y} \rightarrow \mathcal{X}$. We denote by $\iota_{\mathcal{X}}: \mathcal{X} \rightarrow \widetilde{\mathfrak{g}}^{(1)}$ and $\iota \mathcal{Y}: \mathcal{Y} \rightarrow S_{\alpha}^{(1)}$ the inclusion morphisms (which are flat). If $\mathcal{F}$ is in $\operatorname{Coh}\left(\widetilde{\mathfrak{g}}^{(1)}\right)$, then $\left(\iota_{\mathcal{X}}\right)^{*} \mathcal{F}$ is just the completion of $\mathcal{F}$ along $\mathcal{B}_{\chi}^{(1)}$ (see [EGA I, 10.8.8]), and similarly for $\mathcal{Y}$. Recall the vector bundles $\mathcal{M}_{(\nu, \chi)}^{\mathcal{B}}$ on $\mathcal{X}$ (for $\nu \in \mathbb{X}$ regular) introduced in Remark 5.1 .2 (ii). Then by definition, for $\mathcal{F}$ in $\mathcal{D}^{b} \operatorname{Coh}_{\mathcal{B}_{\chi}^{(1)}}\left(\widetilde{\mathfrak{g}}^{(1)}\right)$,

$$
\gamma_{(\nu, \chi)}^{\mathcal{B}}(\mathcal{F}) \cong R \Gamma\left(\mathcal{M}_{(\nu, \chi)}^{\mathcal{B}} \otimes \mathcal{X}\left(\iota_{\mathcal{X}}\right)^{*} \mathcal{F}\right)
$$

Let us also remark that by BMR2, 2.2.3(c)] and the choice of vector bundles we have a functorial isomorphism

$$
\left(\mathbf{I}_{(\lambda, \chi)}^{\alpha}\right)^{-1} \circ \gamma_{(\lambda, \chi)}^{\mathcal{B}} \cong \gamma_{\left(s_{\alpha} \bullet \lambda, \chi\right)}^{\mathcal{B}} .
$$

Now let $\mathcal{F} \in \mathcal{D}^{b} \operatorname{Coh}_{\mathcal{B}_{\chi}^{(1)}}\left(\widetilde{\mathfrak{g}}^{(1)}\right)$. For simplicity, we write $(*)$ for the object $\gamma_{(\lambda, \chi)}^{\mathcal{B}} \circ$ $F_{\widetilde{\mathfrak{g}}^{(1)} \rightarrow \widetilde{\mathfrak{g}}^{(1)}}^{\mathcal{O}_{S^{(1)}}(-\rho, \rho-\alpha)}(\mathcal{F})$. By definition and EGA III $\left.1,4.1 .5\right]$, we have functorial isomorphisms

$$
\begin{aligned}
(*) & \cong R \Gamma\left(\mathcal{M}_{(\lambda, \chi)}^{\mathcal{B}} \otimes_{\mathcal{X}}\left(\iota_{\mathcal{X}}\right)^{*} R\left(q_{2}\right)_{*}\left(L\left(q_{1}\right)^{*} \mathcal{F} \otimes_{S_{\alpha}^{(1)}} \mathcal{O}_{S_{\alpha}^{(1)}}(-\rho, \rho-\alpha)\right)\right) \\
& \cong R \Gamma\left(\mathcal{M}_{(\lambda, \chi)}^{\mathcal{B}} \otimes_{\mathcal{X}} R\left(\widehat{q_{2}}\right)_{*}\left((\iota \mathcal{Y})^{*} L\left(q_{1}\right)^{*} \mathcal{F} \otimes_{\mathcal{Y}} \mathcal{O}_{\mathcal{Y}}(-\rho, \rho-\alpha)\right)\right) .
\end{aligned}
$$

Now, as $q_{1} \circ \iota \mathcal{Y}=\iota \mathcal{X} \circ \widehat{q_{1}}$, we deduce that

$$
(*) \cong R \Gamma\left(\mathcal{M}_{(\lambda, \chi)}^{\mathcal{B}} \otimes \mathcal{X} R\left(\widehat{q_{2}}\right)_{*}\left(L\left(\widehat{q_{1}}\right)^{*}\left(\iota_{\mathcal{X}}\right)^{*} \mathcal{F} \otimes \mathcal{Y} \mathcal{O}_{\mathcal{Y}}(-\rho, \rho-\alpha)\right)\right) .
$$

By the projection formula applied to $\widehat{q_{2}}$, we then have

$$
\begin{aligned}
(*) & \cong R \Gamma \circ R\left(\widehat{q_{2}}\right)_{*}\left(\left(\widehat{q_{2}}\right)^{*} \mathcal{M}_{(\lambda, \chi)}^{\mathcal{B}} \otimes_{\mathcal{Y}} L\left(\widehat{q_{1}}\right)^{*}\left(\iota_{\mathcal{X}}\right)^{*} \mathcal{F} \otimes_{\mathcal{Y}} \mathcal{O}_{\mathcal{Y}}(-\rho, \rho-\alpha)\right) \\
& \cong R \Gamma \circ R\left(\widehat{q_{1}}\right)_{*}\left(\left(\widehat{q_{2}}\right)^{*} \mathcal{M}_{(\lambda, \chi)}^{\mathcal{B}} \otimes_{\mathcal{Y}} L\left(\widehat{q_{1}}\right)^{*}\left(\iota_{\mathcal{X}}\right)^{*} \mathcal{F} \otimes_{\mathcal{Y}} \mathcal{O}_{\mathcal{Y}}(-\rho, \rho-\alpha)\right) .
\end{aligned}
$$

Finally, the projection formula applied to $\widehat{q_{1}}$ gives

$$
(*) \cong R \Gamma\left((\iota \mathcal{X})^{*} \mathcal{F}^{L} \otimes \mathcal{X} R\left(\widehat{q_{1}}\right)_{*}\left(\left(\widehat{q_{2}}\right)^{*} \mathcal{M}_{(\lambda, \chi)}^{\mathcal{B}} \otimes_{\mathcal{Y}} \mathcal{O}_{\mathcal{Y}}(-\rho, \rho-\alpha)\right)\right)
$$


It follows from (5.4.5) and (5.4.6) that it is enough, to prove isomorphism (5.4.2), to construct an isomorphism

$$
R\left(\widehat{q_{1}}\right)_{*}\left(\left(\widehat{q}_{2}\right)^{*} \mathcal{M}_{(\lambda, \chi)}^{\mathcal{B}} \otimes \mathcal{Y} \mathcal{O}_{\mathcal{Y}}(-\rho, \rho-\alpha)\right) \cong \mathcal{M}_{\left(s_{\alpha} \bullet \lambda, \chi\right)}^{\mathcal{B}}
$$

in the derived category of coherent sheaves on $\mathcal{X}$. Let $\mathcal{I}$ be the ideal of definition of $\mathcal{B}_{\chi}^{(1)}$ in $\widetilde{\mathfrak{g}}^{(1)}$. By [EGA I, 10.11.3] and EGA III $\left.1,3.4 .3\right]$, it is enough to show that for all $n \geq 1$ we have an isomorphism

$$
\left(\mathcal{O}_{\mathcal{X}} / \mathcal{I}^{n}\right)^{L} \otimes_{\mathcal{X}} R\left(\widehat{q_{1}}\right)_{*}\left(\left(\widehat{q_{2}}\right)^{*} \mathcal{M}_{(\lambda, \chi)}^{\mathcal{B}} \otimes_{\mathcal{Y}} \mathcal{O}_{\mathcal{Y}}(-\rho, \rho-\alpha)\right) \cong\left(\mathcal{O}_{\mathcal{X}} / \mathcal{I}^{n}\right)^{\stackrel{L}{\otimes}} \mathcal{X} \mathcal{M}_{\left(s_{\alpha} \bullet \lambda, \chi\right)}^{\mathcal{B}} .
$$

Using isomorphisms (5.4.5) and (5.4.6), and the fact that $R \Gamma$ is an equivalence of categories, this isomorphism follows easily from Lemma 5.4.3 applied to $\mathcal{O}_{\mathcal{X}} / \mathcal{I}^{n}$.

Remark 5.4.7. In [B2], Bezrukavnikov explains the importance of this action of $B_{\text {aff }}^{\prime}$ in his plan of proof of Lusztig's conjecture concerning the representation theory of $\mathfrak{g}$. There, the definition of $S_{\alpha}$ is different from ours, but of course they are equivalent (i.e. they define the same subscheme of $\widetilde{\mathfrak{g}} \times \mathfrak{\mathfrak { g }}$ ). He also considers the action on $\mathcal{D}^{b} \operatorname{Coh}(\widetilde{\mathcal{N}})$ (see [B2, theorem 2.1]), without giving a proof of its existence.

\section{RELATION TO REPRESENTATION THEORY IN CHARACTERISTIC ZERO}

In this section we establish a connection between our constructions in the case $\mathbb{k}=\mathbb{C}$ and Ginzburg's description of the equivariant K-theory of the Steinberg variety. We also relate them to Springer's action of the Weyl group on the homology of a Springer fiber.

As above, we assume $G$ has no component of type $\mathbf{G}_{2}$, and we take $\mathbb{k}=\mathbb{C}$.

6.1. Equivariant K-theory of the Steinberg variety. First we need a result which is analogous to Corollary 5.3.2, but for the action on $\mathcal{D}^{b} \operatorname{Coh}(\widetilde{\mathcal{N}})$. It is valid over any algebraically closed field $\mathbb{k}$. Consider the variety $S_{\alpha}^{\prime}$. Geometrically, it can be described as:

$$
S_{\alpha}^{\prime}=\left\{\left(X, g_{1} B, g_{2} B\right) \in \mathfrak{g}^{*} \times \mathcal{B} \times \mathcal{B} \mid X_{\mid g_{1} \cdot \mathfrak{b}+g_{2} \cdot \mathfrak{b}}=0\right\} .
$$

It has two irreducible components. One is $\Delta \widetilde{\mathcal{N}}$, the diagonal embedding of $\widetilde{\mathcal{N}}$, and the other one is

$$
Y_{\alpha}:=\left\{\left(X, g_{1} B, g_{2} B\right) \in \mathfrak{g}^{*} \times\left(\mathcal{B} \times \mathcal{P}_{\alpha} \mathcal{B}\right) \mid X_{\mid g_{1} \cdot \mathfrak{p}_{\alpha}}=0\right\},
$$

which is a vector bundle on $\mathcal{B} \times \mathcal{P}_{\alpha} \mathcal{B}$, of $\operatorname{rank} \operatorname{dim}(\mathfrak{g} / \mathfrak{b})-1$.

Lemma 6.1.1. There exist exact sequences of quasi-coherent sheaves, where the surjections are restriction maps:

$$
\begin{aligned}
\mathcal{O}_{\Delta \widetilde{\mathcal{N}}} \hookrightarrow \mathcal{O}_{S_{\alpha}^{\prime}}(\rho-\alpha,-\rho) \rightarrow \mathcal{O}_{Y_{\alpha}}(\rho-\alpha,-\rho), & \mathcal{O}_{Y_{\alpha}}(\rho-\alpha,-\rho) \hookrightarrow \mathcal{O}_{S_{\alpha}^{\prime}} \rightarrow \mathcal{O}_{\Delta \widetilde{\mathcal{N}}} .
\end{aligned}
$$

Proof. The construction of the exact sequences is analogous to that in Lemma 5.3.1. Let us introduce the following subvarieties of $\mathfrak{g}^{*} \times\left(P_{\alpha} / B\right)$ :

$$
\begin{gathered}
\mathscr{D}_{\alpha}^{\prime}:=(\mathfrak{g} / \mathfrak{b})^{*} \times(B / B), \\
\mathscr{S}_{\alpha}^{\prime}:=\left\{(X, g B) \in \mathfrak{g}^{*} \times\left(P_{\alpha} / B\right) \mid X_{\mid \mathfrak{b}+g \cdot \mathfrak{b}}=0\right\}, \\
\mathscr{Y}_{\alpha}:=\left\{(X, g B) \in \mathfrak{g}^{*} \times\left(P_{\alpha} / B\right) \mid X_{\mid \mathfrak{p}_{\alpha}}=0\right\} .
\end{gathered}
$$

Then we have isomorphisms $\Delta \widetilde{\mathcal{N}} \cong G \times{ }^{B} \mathscr{D}_{\alpha}^{\prime}, S_{\alpha}^{\prime} \cong G \times{ }^{B} \mathscr{S}_{\alpha}^{\prime}, Y_{\alpha} \cong G \times{ }^{B} \mathscr{Y}_{\alpha}$. Let us recall the equations of the varieties $\mathscr{D}_{\alpha}^{\prime}, \mathscr{S}_{\alpha}^{\prime}, \mathscr{Y}_{\alpha}$. We use the affine covering 
$\left(P_{\alpha} / B\right)=\left(U_{\alpha} B / B\right) \cup\left(s_{\alpha} U_{\alpha} B / B\right)$, and the isomorphisms induced by $u_{\alpha}$, respectively by $t \mapsto n_{\alpha} u_{\alpha}(t): \mathbb{k} \cong U_{\alpha} B / B, \mathbb{k} \cong s_{\alpha} U_{\alpha} B / B$. As coordinates on $\mathfrak{g}^{*}$ we use the basis $\left\{e_{\gamma}, \gamma \in R, h_{\beta}, \beta \in \Phi\right\}$ of $\mathfrak{g}$. Then we can deduce from the computations in section 4 the equations defining $\left.\mathscr{S}_{\alpha}^{\prime}\right|_{U_{\alpha} B / B},\left.\mathscr{D}_{\alpha}^{\prime}\right|_{U_{\alpha} B / B}$ and $\left.\mathscr{Y}_{\alpha}\right|_{U_{\alpha} B / B}$ as closed suvarieties of $\mathfrak{g}^{*} \times \mathbb{k}$. Namely, these three varieties are defined by the equations $e_{\gamma}$ $\left(\gamma \in R^{-}\right), h_{\beta}(\beta \in \Phi)$ and, respectively, $t e_{\alpha}, t, e_{\alpha}$. Hence there are exact sequences

$$
\begin{aligned}
& \mathbb{k}\left[\left.\mathscr{D}_{\alpha}^{\prime}\right|_{U_{\alpha} B / B}\right] \hookrightarrow \mathbb{k}\left[\left.\mathscr{S}_{\alpha}^{\prime}\right|_{U_{\alpha} B / B}\right] \rightarrow \mathbb{k}\left[\left.\mathscr{Y}_{\alpha}\right|_{U_{\alpha} B / B}\right], \\
& \mathbb{k}\left[\left.\mathscr{Y}_{\alpha}\right|_{U_{\alpha} B / B}\right] \hookrightarrow \mathbb{k}\left[\left.\mathscr{S}_{\alpha}^{\prime}\right|_{U_{\alpha} B / B}\right] \rightarrow \mathbb{k}\left[\left.\mathscr{D}_{\alpha}^{\prime}\right|_{U_{\alpha} B / B}\right],
\end{aligned}
$$

where the first maps are respectively the multiplication by $e_{\alpha}$ and $t$.

Over $s_{\alpha} U_{\alpha} B / B$ we have $\left.\mathscr{D}_{\alpha}^{\prime}\right|_{s_{\alpha} U_{\alpha} B / B}=\emptyset,\left.\mathscr{S}_{\alpha}^{\prime}\right|_{s_{\alpha} U_{\alpha} B / B}=\left.\mathscr{Y}_{\alpha}\right|_{s_{\alpha} U_{\alpha} B / B}$. Under the change of coordinates $t$ is sent to $-\frac{1}{t}$, and $e_{\alpha}$ to 0 . Hence there are exact sequences of quasi-coherent sheaves

$$
\mathcal{O}_{\mathscr{D}_{\alpha}^{\prime}} \hookrightarrow \mathcal{O}_{\mathscr{S}_{\alpha}^{\prime}} \rightarrow \mathcal{O}_{\mathscr{Y}_{\alpha}}, \quad \mathcal{O}_{\mathscr{Y}_{\alpha}} \otimes_{\mathcal{O}_{P_{\alpha} / B}} \mathcal{O}_{P_{\alpha} / B}(-\rho) \hookrightarrow \mathcal{O}_{\mathscr{S}_{\alpha}^{\prime}} \rightarrow \mathcal{O}_{\mathscr{D}_{\alpha}^{\prime}} .
$$

Concerning the $B$-equivariant structure, we remark that the second exact sequence was constructed just like in Lemma 5.3.1. Hence, as there we have an exact sequence of $B$-equivariant sheaves

$$
\mathcal{O}_{\mathscr{Y}_{\alpha}}(-\rho) \otimes_{\mathbb{k}} \mathbb{k}_{B}(\rho-\alpha) \hookrightarrow \mathcal{O}_{\mathscr{S}_{\alpha}^{\prime}} \rightarrow \mathcal{O}_{\mathscr{D}_{\alpha}^{\prime}} .
$$

Inducing from $B$ to $G$ we obtain the second exact sequence of the lemma. Concerning the first exact sequence, its first arrow is given by the multiplication by $e_{\alpha}$, which has weight $\alpha$ for the action of $B$. Hence the $B$-equivariant exact sequence reads

$$
\mathcal{O}_{\mathscr{D}_{\alpha}^{\prime}} \otimes_{\mathbb{k}} \mathbb{k}_{B}(\alpha) \hookrightarrow \mathcal{O}_{\mathscr{S}_{\alpha}^{\prime}} \rightarrow \mathcal{O}_{\mathscr{Y}_{\alpha}} .
$$

Inducing, we obtain $\mathcal{O}_{\Delta \widetilde{\mathcal{N}}}(\alpha, 0) \hookrightarrow \mathcal{O}_{S_{\alpha}^{\prime}} \rightarrow \mathcal{O}_{Y_{\alpha}}$. Now $\mathcal{O}_{\mathcal{B} \times_{\mathcal{P}_{\alpha}} \mathcal{B}}(-\rho, \rho)$ is trivial on the diagonal. Hence we also have

$$
\mathcal{O}_{\Delta \widetilde{\mathcal{N}}}(\alpha-\rho, \rho) \hookrightarrow \mathcal{O}_{S_{\alpha}^{\prime}} \rightarrow \mathcal{O}_{Y_{\alpha}} .
$$

Tensoring by the inverse image of $\mathcal{O}_{\mathcal{B} \times \mathcal{P}_{\alpha} \mathcal{B}}(\rho-\alpha,-\rho)$, we obtain the first exact sequence of the lemma.

Let us define a $\mathbb{C}^{\times}$-action on $\widetilde{\mathcal{N}}$, setting

$$
t \cdot(X, g B):=\left(t^{-2} X, g B\right) .
$$

This action commutes with the natural action of $G$ on $\tilde{\mathcal{N}}$. We denote by

$$
\langle 1\rangle: \mathcal{D}^{b} \operatorname{Coh}^{G \times \mathbb{C}^{\times}}(\widetilde{\mathcal{N}}) \rightarrow \mathcal{D}^{b} \operatorname{Coh}^{G \times \mathbb{C}^{\times}}(\tilde{\mathcal{N}})
$$

the tensor product with the one-dimensional $\mathbb{C}^{\times}$-module given by $\operatorname{Id}_{\mathbb{C}^{\times}}$, and similarly for any variety with a $\mathbb{C}^{\times}$-action. Then the exact sequences of Lemma 6.1.1 have $G \times \mathbb{C}^{\times}$-equivariant versions

$$
\begin{gathered}
\mathcal{O}_{\Delta \widetilde{\mathcal{N}}}\langle 2\rangle \hookrightarrow \mathcal{O}_{S_{\alpha}^{\prime}}(\rho-\alpha,-\rho) \rightarrow \mathcal{O}_{Y_{\alpha}}(\rho-\alpha,-\rho), \\
\mathcal{O}_{Y_{\alpha}}(\rho-\alpha,-\rho) \hookrightarrow \mathcal{O}_{S_{\alpha}^{\prime}} \rightarrow \mathcal{O}_{\Delta \widetilde{\mathcal{N}}} .
\end{gathered}
$$

If $H$ is an algebraic group (over $\mathbb{C}$ ) acting on a variety $X$, we denote by $K_{H}(X)$ the $H$-equivariant K-theory of $X$. This is by definition the Grothendieck group of the category $\mathrm{Coh}^{H}(X)$ of $H$-equivariant coherent sheaves on $X$, or of its derived category $\mathcal{D}^{b} \mathrm{Coh}^{H}(X)$. We refer to [L3, section 6] for generalities on equivariant K-theory, and to [B1, section 2] and [CG, 5.1] for the main properties of derived 
categories of equivariant coherent sheaves. If $\mathcal{F}$ is an object of $\mathcal{D}^{b} \operatorname{Coh}^{H}(X)$, we denote by $[\mathcal{F}]$ its image in $K_{H}(X)$.

Let $\mathcal{N}$ be the variety of nilpotent elements in $\mathfrak{g}^{*}$. We have the Springer resolution $\pi: \widetilde{\mathcal{N}} \rightarrow \mathcal{N}$. We will be interested in the Steinberg variety

$$
Z:=\tilde{\mathcal{N}} \times_{\mathcal{N}} \tilde{\mathcal{N}}
$$

and more precisely to the group $K_{G \times \mathbb{C} \times}(Z)$. First, let us describe the ring structure on this K-group. There is a natural closed embedding $j: Z \hookrightarrow \widetilde{\mathcal{N}}^{2}$. Let $p_{a, b}: \widetilde{\mathcal{N}}^{3} \rightarrow \tilde{\mathcal{N}}^{2}$ denote the projection to the $a$-th and $b$-th factors $(1 \leq a<b \leq 3)$. If $\mathcal{F}$ and $\mathcal{G}$ are in $\mathcal{D}^{b} \mathrm{Coh}^{G \times \mathbb{C}^{\times}}(Z)$, then $R\left(p_{1,3}\right)_{*}\left(p_{1,2}^{*}\left(j_{*} \mathcal{F}\right){\stackrel{\mathscr{\otimes}}{\mathbb{\mathcal { N }}^{3}}} p_{2,3}^{*}\left(j_{*} \mathcal{G}\right)\right)$ is only in $\mathcal{D}^{b} \mathrm{Coh}^{G \times \mathbb{C}^{\times}}\left(\widetilde{\mathcal{N}}^{2}\right)$, but its cohomology is supported on $Z$. Hence the class $\left[R\left(p_{1,3}\right)_{*}\left(p_{1,2}^{*}\left(j_{*} \mathcal{F}\right) \stackrel{L}{\otimes} \widetilde{\mathcal{N}}^{3} p_{2,3}^{*}\left(j_{*} \mathcal{G}\right)\right)\right]$ is a well-defined element of $K_{G \times \mathbb{C}^{\times}}(Z)$ (see [B1, 2. Lemma 3(b)], [L3, 6.2]). The ring structure on $K_{G \times \mathbb{C} \times}(Z)$ is then given by the product:

$$
[\mathcal{F}] \cdot[\mathcal{G}]:=\left[R\left(p_{1,3}\right)_{*}\left(p_{1,2}^{*}\left(j_{*} \mathcal{F}\right) \stackrel{L}{\otimes}_{\widetilde{\mathcal{N}}^{3}} p_{2,3}^{*}\left(j_{*} \mathcal{G}\right)\right)\right]
$$

Note that the unit for this product is $\left[\mathcal{O}_{\Delta \widetilde{\mathcal{N}}}\right]$.

Let $v$ be an indeterminate, and $\mathcal{A}:=\mathbb{Z}\left[v, v^{-1}\right]$. Let $\mathcal{H}$ be the extended affine Hecke algebra associated to $R$ (over $\mathcal{A}$ ). Using the Bernstein presentation (see e.g. L3. 1.19]) one sees that $\mathcal{H}$ is the quotient of $\mathcal{A}\left[B_{\text {aff }}^{\prime}\right]$, the group algebra of $B_{\text {aff }}^{\prime}$ over $\mathcal{A}$, by the ideal generated by the relations

$$
\left(T_{\alpha}+v^{-1}\right)\left(T_{\alpha}-v\right)=0
$$

for $\alpha \in \Phi$. We let $\mathcal{A}$ act on $K_{G \times \mathbb{C} \times}(Z)$ by setting $v \cdot[\mathcal{F}]:=[\mathcal{F}\langle 1\rangle]$. The varieties $Y_{\alpha}$ and $S_{\alpha}^{\prime}$ are $G \times \mathbb{C}^{\times}$-stable subvarieties of $Z$, hence define natural classes $\left[\mathcal{O}_{Y_{\alpha}}\right]$, $\left[\mathcal{O}_{S_{\alpha}^{\prime}}\right]$ in $K_{G \times \mathbb{C}^{\times}}(Z)$. If $x$ and $y$ are in $\mathbb{X}$, the line bundle $\mathcal{O}_{Z}(x, y)$ (see 1.4 for the notation) is naturally an object of $\operatorname{Coh}^{G \times \mathbb{C}^{\times}}(Z)$ (with trivial $\mathbb{C}^{\times}$-action).

As an easy consequence of our results we obtain:

Proposition 6.1.5. The assignment

$$
\left\{\begin{aligned}
T_{\alpha} & \mapsto-v^{-1}\left[\mathcal{O}_{Y_{\alpha}}(-\rho, \rho-\alpha)\right]-v^{-1}=-v^{-1}\left[\mathcal{O}_{S_{\alpha}^{\prime}}\right], \\
\theta_{x} & \mapsto\left[\mathcal{O}_{\Delta \widetilde{\mathcal{N}}}(x)\right]
\end{aligned}\right.
$$

extends to a morphism of $\mathcal{A}$-algebras $\mathcal{H} \rightarrow K_{G \times \mathbb{C} \times}(Z)$.

Remark 6.1.6. This result is well known (see e.g. [L3, 7.25] or [CG, 7.6.9]), and this morphism is in fact an isomorphism, as proved in [L3, 8.6] or [CG, 7.6.10]. The construction of this morphism is one of the main steps of the proof of the isomorphism $\mathcal{H} \cong K_{G \times \mathbb{C}^{\times}}(Z)$ (both for the proof by Ginzburg, see [G2] or [CG], and for the alternate proof by Lusztig, see [L3]). These previous constructions are indirect, using an action on a module to prove the fact that the image of the generators satisfy the relations of $\mathcal{H}$. Using our constructions, one can give a direct proof of the relations in $K_{G \times \mathbb{C} \times}(Z)$ (using no K-theoretic result). Moreover, this proof gives a more concrete interpretation of the image of the generators $T_{\alpha}$; namely, this image is a multiple of the class of $\mathcal{O}_{S_{\alpha}^{\prime}}$.

Proof. First, the equality

$$
-v^{-1}\left[\mathcal{O}_{Y_{\alpha}}(-\rho, \rho-\alpha)\right]-v^{-1}=-v^{-1}\left[\mathcal{O}_{S_{\alpha}^{\prime}}\right]
$$


follows from the exact sequence (6.1.3). We have to check that the elements $-v^{-1}\left[\mathcal{O}_{S_{\alpha}^{\prime}}\right]$ for $\alpha \in \Phi$ and $\left[\mathcal{O}_{\Delta \widetilde{\mathcal{N}}}(x)\right]$ for $x \in \mathbb{X}$ satisfy relations (1) to (4) of Theorem 1.1.3, and the quadratic relations (6.1.4).

Relation (2) is trivial, and relations (1) and (3) follow from the results of section 4. Now the exact sequences of Lemma 1.5.5 admit the following $\mathbb{C}^{\times}$-equivariant versions (where the action on $\widetilde{\mathfrak{g}}$ is the natural one, extending the action on $\widetilde{\mathcal{N}}$ ):

$$
\begin{aligned}
& \mathcal{O}_{V_{\alpha}^{1}}\langle 2\rangle \hookrightarrow \mathcal{O}_{V_{\alpha}}(\rho-\alpha,-\rho, 0) \rightarrow \mathcal{O}_{V_{\alpha}^{2}}(\rho-\alpha,-\rho, 0), \\
& \mathcal{O}_{V_{\alpha}^{1}}\langle 2\rangle \hookrightarrow \mathcal{O}_{V_{\alpha}}(0,-\rho, \rho-\alpha) \rightarrow \mathcal{O}_{V_{\alpha}^{2}}(0,-\rho, \rho-\alpha) .
\end{aligned}
$$

We deduce as in section 4 that $-v^{-1}\left[\mathcal{O}_{S_{\alpha}^{\prime}}\right]$ is invertible, and

$$
\left(-v^{-1}\left[\mathcal{O}_{S_{\alpha}^{\prime}}\right]\right)^{-1}=-v^{-1}\left[\mathcal{O}_{S_{\alpha}^{\prime}}(\rho-\alpha,-\rho)\right] .
$$

Then relation (4) is easy to prove (as in 1.6).

Finally, for the quadratic relations, consider the exact sequence (6.1.2). It yields

$$
-v^{-1}\left[\mathcal{O}_{S_{\alpha}^{\prime}}(\rho-\alpha,-\rho)\right]=-v^{-1}\left[\mathcal{O}_{Y_{\alpha}}(\rho-\alpha,-\rho)\right]-v .
$$

Using relations (6.1.7) and (6.1.8), we deduce from (6.1.9) that

$$
\left(-v^{-1}\left[\mathcal{O}_{S_{\alpha}^{\prime}}\right]\right)^{-1}=\left(-v^{-1}\left[\mathcal{O}_{S_{\alpha}^{\prime}}\right]\right)+\left(v^{-1}-v\right) .
$$

This is equivalent to relation (6.1.4).

It follows from these considerations that the natural action of $\mathcal{H}$ on $K_{G \times \mathbb{C} \times}(\tilde{\mathcal{N}})$ (see [CG, 7.6.6]) can be lifted to an action of $B_{\text {aff }}^{\prime}$ on the category $\mathcal{D}^{b} \mathrm{Coh}^{G \times \mathbb{C}^{\times}}(\widetilde{\mathcal{N}})$.

Remark 6.1.10. Let $\chi \in \mathfrak{g}^{*}$ be nilpotent, and let $\mathcal{B}_{\chi}$ be the corresponding Springer fiber, i.e., the inverse image of $\chi$ under $\widetilde{\mathfrak{g}} \rightarrow \mathfrak{g}^{*}$ (see 5.1 note however that now we work over $\mathbb{C}$ ). Let $M$ be a closed subgroup of the stabilizer of $\chi$ in $G \times \mathbb{C}^{\times}$, for the action defined by $(g, z) \cdot \chi=z^{-2} g \cdot \chi$. Then $M$ stabilizes $\mathcal{B}_{\chi} \subset \tilde{\mathcal{N}}$. Our constructions yield an action of $B_{\text {aff }}^{\prime}$ on $\mathcal{D}^{b} \operatorname{Coh}^{M}(\widetilde{\mathcal{N}})$, which stabilizes the full subcategory $\mathcal{D}^{b} \operatorname{Coh}_{\mathcal{B}_{\chi}}^{M}(\tilde{\mathcal{N}})$ of complexes whose cohomology sheaves are supported on $\mathcal{B}_{\chi}$. The Grothendieck group of the category $\mathcal{D}^{b} \operatorname{Coh}_{\mathcal{B}_{\chi}}^{M}(\tilde{\mathcal{N}})$ identifies with $K_{M}\left(\mathcal{B}_{\chi}\right)$. The same considerations as above show that the action of $B_{\text {aff }}^{\prime}$ induces an action of $\mathcal{H}$ on $K_{M}\left(\mathcal{B}_{\chi}\right)$. This is the action considered in [L5, 3.4]. In [L5], Lusztig explains the importance of these modules in the construction of all the irreducible $\mathcal{H}$-modules over $\mathbb{C}$.

6.2. Springer's representations of $W$. Now we consider Springer's representations of the finite Weyl group. More precisely we follow Ginzburg's approach to this question in G1] (see [CG, chapter 3] for the same arguments, in the framework of homology rather than K-theory).

As in 6.1, our constructions yield a $\mathbb{Z}$-algebra morphism

$$
\mathbb{Z}\left[B_{0}\right] \rightarrow K(Z),
$$

where $K(Z)$ is the non-equivariant K-theory of the Steinberg variety $Z$, and $B_{0}$ is the finite braid group (see 1.1 for the definition). The exact sequences of Lemma 6.1.1 show that for $\alpha \in \Phi$ the image of $\left(T_{\alpha}\right)^{2}$ in $K(Z)$ is 1 . Hence the previous morphism gives a morphism

$$
\mathbb{Z}[W] \rightarrow K(Z)
$$


Following Ginzburg, we consider $K(Z)$ as the Grothendieck group of the category $\operatorname{Coh}_{Z}(\widetilde{\mathcal{N}} \times \widetilde{\mathcal{N}})$, and denote by $L(Z)$ the quotient by the subgroup generated by the elements $[\mathcal{F}]$ for $\mathcal{F}$ in $\operatorname{Coh}_{Z}(\widetilde{\mathcal{N}} \times \widetilde{\mathcal{N}})$ such that $\operatorname{dim}(\operatorname{Supp}(\mathcal{F}))<\operatorname{dim}(Z)$. Composing the previous morphism with the natural quotient $K(Z) \rightarrow L(Z)$ we obtain a morphism

$$
\mathbb{Z}[W] \rightarrow L(Z) .
$$

The following proposition follows directly from our constructions and the definition of specialization in K-theory as in [CG. 5.3] (use the definition of $S_{\alpha}^{\prime}$ as the intersection $\left.S_{\alpha} \cap(\widetilde{\mathcal{N}} \times \widetilde{\mathcal{N}})\right)$.

Proposition 6.2.2. The morphism (6.2.1) coincides with the isomorphism of G1, $5.3]: \mathbb{Z}[W] \stackrel{\sim}{\longrightarrow} L(Z)$.

This isomorphism is the main step in Ginzburg's approach to Springer's construction of the representations of $W$ on the top homology of Springer fibers (see CG, 3.5-6]). Choose a nilpotent $\chi \in \mathfrak{g}^{*}$, and consider the Springer fiber $\mathcal{B}_{\chi}$ (see 6.1). As noted above, the $B_{\text {aff }}^{\prime}$-action on $\mathcal{D}^{b} \operatorname{Coh}(\widetilde{\mathcal{N}})$ induces an action of $B_{0}$ on $K\left(\mathcal{B}_{\chi}\right)$ (this is the case $M=1 \mathrm{in} \mathrm{Remark} \mathrm{6.1.10}$ ), which factorizes through the finite Weyl group $W$ (for the same reason as above). This in turn induces an action of $W$ on $L\left(\mathcal{B}_{\chi}\right)$, the quotient of $K\left(\mathcal{B}_{\chi}\right)$ defined as above for $L(Z)$. By Grothendieck-Riemann-Roch, we have an isomorphism $L\left(\mathcal{B}_{\chi}\right) \otimes_{\mathbb{Z}} \mathbb{Q} \cong H^{\text {top }}\left(\mathcal{B}_{\chi}, \mathbb{Q}\right)$. Via this isomorphism, the action of $W$ gives Springer's action on $H^{\text {top }}\left(\mathcal{B}_{\chi}, \mathbb{Q}\right.$ ) (by Proposition 6.2.2 and CG, $3.5-6])$.

\section{Appendix A. Presentation of $B_{\text {aff }}^{\prime}$ (Joint with Roman Bezrukavnikov)}

In this appendix we prove Theorem 1.1.3. We understand that this theorem was known to Lusztig, although he did not publish a proof.

The facts that the elements $T_{\alpha}$ and $\theta_{x}$ generate $B_{\text {aff }}^{\prime}$, and satisfy the relations of the theorem, are proved in [L2, 2.7, 2.8]. We denote by $\hat{B}$ the group with the given presentation. There exists a (surjective 8 ) morphism $\psi: \hat{B} \rightarrow B_{\text {aff. }}^{\prime}$. To prove the theorem we construct an inverse $\phi$ to this morphism. To avoid confusion, in this appendix we denote by $\hat{T}_{\alpha}$ and $\hat{\theta}_{x}$ the images of the generators in $\hat{B}$. Hence we have $\psi\left(\hat{T}_{\alpha}\right)=T_{\alpha}, \psi\left(\hat{\theta}_{x}\right)=\theta_{x}$.

Acknowledgement. We thank George Lusztig for suggesting Theorem 1.1.3 to one of us.

A.1. A second "length function". In this paragraph we introduce a second "length function" on $W_{\text {aff }}^{\prime}$, denoted $L$, with values in $\mathbb{Z}$. Recall the notation of 1.1 . Let $\mathscr{H}$ by the set of reflection hyperplanes of $W_{\text {aff }}$ in $\mathbb{X} \otimes \mathbb{R}$, and $\mathscr{A}$ be the set of alcoves. Let $C_{0}$ be the fundamental chamber, i.e., $C_{0}=\{x \in \mathbb{X} \otimes \mathbb{R} \mid \forall \alpha \in$ $\left.\Phi,\left\langle x, \alpha^{\vee}\right\rangle \geq 0\right\}$. If $H \in \mathscr{H}$, we denote by $E_{H}^{+}$the half space defined by $H$ that intersects all translates of $C_{0}$, and by $E_{H}^{-}$the other half space. Then, following Jantzen and Lusztig (see [L1]) we introduce the function $d$ on $\mathscr{A}^{2}$, defined by

$$
\begin{aligned}
d(A, B)=\#\left\{H \in \mathscr{H} \mid A \subset E_{H}^{-} \text {and } B \subset E_{H}^{+}\right\} & \\
& -\#\left\{H \in \mathscr{H} \mid A \subset E_{H}^{+} \text {and } B \subset E_{H}^{-}\right\} .
\end{aligned}
$$

${ }^{8}$ We do not use this surjectivity in our proof, but rather reprove it. 
It is clear from the definition that $d(A, B)=-d(B, A)$. Moreover, $d$ satisfies the following formula for three alcoves $A, B$ and $C$ (see [L1, 1.4.1]):

$$
d(A, B)+d(B, C)+d(C, A)=0 .
$$

Now we can define the function $L$ on $W_{\text {aff }}^{\prime}$ by setting

$$
L(w):=d\left(A_{0}, w^{-1} A_{0}\right)
$$

(recall that $A_{0}$ denotes the fundamental alcove). For $w \in W$ we have $L(w)=-\ell(w)$, and for $x \in \mathbb{X}$ antidominant we have $L\left(t_{x}\right)=\ell\left(t_{x}\right)$. Similarly, if $x$ is dominant we have $L\left(t_{x}\right)=-\ell\left(t_{x}\right)$. Moreover, $|L(w)| \leq \ell(w)$ for any $w \in W_{\text {aff }}^{\prime}$ (for all of this, use $[\mathrm{Hu}, 4.5])$.

Lemma A.1.2. For any $u, w \in W_{\text {aff }}^{\prime}$, we have $|L(w u)-L(u)| \leq \ell(w)$. Moreover, for any $w \in W_{\text {aff }}^{\prime}$ there exists $u \in W_{\text {aff }}^{\prime}$ such that $L(w u)-L(u)=-\ell(w)$.

Proof. Using formula A.1.1 we have

$$
L(w u)-L(u)=d\left(A_{0}, u^{-1} w^{-1} A_{0}\right)-d\left(A_{0}, u^{-1} A_{0}\right)=d\left(u^{-1} A_{0}, u^{-1} w^{-1} A_{0}\right) .
$$

Hence $|L(w u)-L(u)|$ is at most the number of hyperplanes in $\mathscr{H}$ separating $u^{-1} A_{0}$ and $u^{-1} w^{-1} A_{0}$, which equals the number of hyperplanes separating $A_{0}$ and $w^{-1} A_{0}$. This number is precisely $\ell\left(w^{-1}\right)=\ell(w)$.

Let us now consider the second assertion. Let $\xi$ be a point in $A_{0}$. Let $u \in W$ be such that $u^{-1}\left(w^{-1}(\xi)-\xi\right)$ is in $w_{0} C_{0}$, where $w_{0}$ is the longest element of $W$. Then it is clear that $d\left(u^{-1} A_{0}, u^{-1} w^{-1} A_{0}\right)=-\ell(w)$.

A.2. Computations in $W_{\mathrm{aff}}^{\prime}$. In 1.1 we have defined a section $C$ of the morphism $B_{\text {aff }}^{\prime} \rightarrow W_{\text {aff }}^{\prime}$. Now, let us define another section $S: W_{\text {aff }}^{\prime} \rightarrow B_{\text {aff }}^{\prime}$ by setting $S\left(w_{f} \cdot t_{x}\right):=C\left(w_{f}\right) \theta_{x}$ for $w_{f} \in W$ and $x \in \mathbb{X}$, where we have used the isomorphism $W_{\text {aff }}^{\prime} \cong W \ltimes \mathbb{X}$. We will show that one can recover $C$ from $S$.

Lemma A.2.1. Let $u, w \in W_{\text {aff }}^{\prime}$ be such that $L(w u)=L(u)-\ell(w)$. Then we have $S(w u)=C(w) S(u)$.

Proof. First, let us remark that the hypothesis and the conclusion are invariant by replacing $u$ by $u t_{x}$ for some $x \in \mathbb{X}$. Hence we can assume that $u \in W$. We write $w=w_{f} t_{\lambda}$ for some $\lambda \in \mathbb{X}, w_{f} \in W$. Then

$$
L(w u)-L(u)=d\left(u^{-1} A_{0}, u^{-1} w^{-1} A_{0}\right)=d\left(u^{-1} A_{0}, u^{-1}\left(w_{f}\right)^{-1} A_{0}-u^{-1}(\lambda)\right) .
$$

As $u$ and $w_{f}$ are in $W$, and as every hyperplane $H$ between $u^{-1} A_{0}$ and $u^{-1} w^{-1} A_{0}$ is crossed in the direction $E_{H}^{+} \rightsquigarrow E_{H}^{-}$we must have the inequality $\left\langle-u^{-1}(\lambda), \alpha^{\vee}\right\rangle \leq 1$ for any $\alpha \in R^{+}$, i.e., $\left\langle u^{-1}(\lambda), \alpha^{\vee}\right\rangle \geq-1$. Moreover, for any $\alpha \in R^{+}$such that $w_{f} u(\alpha) \in R^{+}$we have $\left\langle u^{-1}(\lambda), \alpha^{\vee}\right\rangle \geq 0$. Indeed, in this case $u^{-1}\left(w_{f}\right)^{-1} A_{0}$ is in $E_{H_{\alpha}}^{+}$for $H_{\alpha}$ the reflection hyperplane of $s_{\alpha}$. Hence if $\left\langle u^{-1}(\lambda), \alpha^{\vee}\right\rangle$ were -1 , then to go from $u^{-1} A_{0}$ to $u^{-1} w^{-1} A_{0}$ we would have to cross $H:=\left\{x \in \mathbb{X} \otimes_{\mathbb{Z}} \mathbb{R} \mid\left\langle x, \alpha^{\vee}\right\rangle=\right.$ $1\}$ in the "wrong" direction (i.e. $E_{H}^{-} \rightsquigarrow E_{H}^{+}$).

Let us write $u^{-1}(\lambda)=\mu_{1}-\mu_{2}$ with $\mu_{1}$ and $\mu_{2}$ dominant weights. We have $w u=$ $\left(w_{f}\right) t_{\lambda} u=w_{f} u t_{u^{-1}(\lambda)}$. Hence $w_{u} t_{\mu_{2}}=w_{f} u t_{\mu_{1}}$. As $\mu_{1}$ is dominant and $w_{f} u \in W$, $\ell\left(w_{f} u t_{\mu_{1}}\right)=\ell\left(w_{f} u\right)+\ell\left(t_{\mu_{1}}\right)$ (see (1.1.1) $)$. Hence $C\left(w_{f} u t_{\mu_{1}}\right)=C\left(w_{f} u\right) C\left(t_{\mu_{1}}\right)$. We will now prove that, also, $\ell\left(w u t_{\mu_{2}}\right)=\ell(w u)+\ell\left(t_{\mu_{2}}\right)$. It will follow that $C\left(w_{u} t_{\mu_{2}}\right)=$ $C(w u) C\left(t_{\mu_{2}}\right)$, and finally that $S(w u)=C(w u)$. 
So, let us prove that $\ell\left(w u t_{\mu_{2}}\right)=\ell(w u)+\ell\left(t_{\mu_{2}}\right)$. Using formula (1.1.1), we have

$$
\begin{gathered}
\ell\left(t_{\mu_{2}}\right)=\sum_{\alpha \in R^{+}}\left\langle\mu_{2}, \alpha^{\vee}\right\rangle, \\
\ell(w u)=\sum_{\substack{\alpha \in R^{+} \\
w_{f} u(\alpha) \in R^{+}}}\left|\left\langle\mu_{1}-\mu_{2}, \alpha^{\vee}\right\rangle\right|+\sum_{\substack{\alpha \in R^{+}, w_{f} u(\alpha) \in R^{-}}}\left|1+\left\langle\mu_{1}-\mu_{2}, \alpha^{\vee}\right\rangle\right|, \\
\ell\left(w_{u} t_{\mu_{2}}\right)=\sum_{\substack{\alpha \in R^{+}, w_{f} u(\alpha) \in R^{+}}}\left\langle\mu_{1}, \alpha^{\vee}\right\rangle+\sum_{\substack{\alpha \in R^{+}, w_{f} u(\alpha) \in R^{-}}}\left(1+\left\langle\mu_{1}, \alpha^{\vee}\right\rangle\right) .
\end{gathered}
$$

We know (see above) that for any $\alpha \in R^{+},\left\langle u^{-1}(\lambda), \alpha^{\vee}\right\rangle \geq-1$, and, for any $\alpha \in R^{+}$ such that $w_{f} u(\alpha) \in R^{+},\left\langle u^{-1}(\lambda), \alpha^{\vee}\right\rangle \geq 0$. The result easily follows.

Finally, we have proved that $S(w u)=C(w u)$. By hypothesis $|L(w u)|=\mid L(u)-$ $\ell(w) \mid=\ell(u)+\ell(w)$ (because $u$ is in $W)$. On the other hand, we have the inequalities $|L(w u)| \leq \ell(w u) \leq \ell(w)+\ell(u)$. We deduce that we must have $\ell(w u)=\ell(w)+\ell(u)$. Hence $C(w u)=C(w) C(u)=C(w) S(u)$. This concludes the proof.

A.3. Computations in $\hat{B}$. The braid group $B_{0}$ is well known to have a presentation with generators the $T_{\alpha}(\alpha \in \Phi)$ and relations (1) of Theorem 1.1.3. Hence there exists a group morphism $\sigma: B_{0} \rightarrow \hat{B}$, which sends $T_{\alpha}$ to $\hat{T}_{\alpha}$. We define $C^{\prime}:=\left.\sigma \circ C\right|_{W}: W \rightarrow \hat{B}$. Then we can define the lift

$$
S^{\prime}: W_{\text {aff }}^{\prime} \rightarrow \hat{B}
$$

by setting $S^{\prime}\left(w_{f} t_{x}\right):=C^{\prime}\left(w_{f}\right) \hat{\theta}_{x}$ for $w_{f} \in W, x \in \mathbb{X}$. The following diagram is commutative:

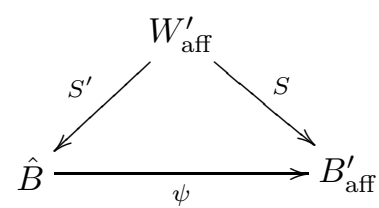

The next proposition is the key step in our proof of Theorem 1.1.3.

Proposition A.3.1. Let $w, u_{1}, u_{2} \in W_{\text {aff }}^{\prime}$ such that $L\left(w u_{1}\right)=L\left(u_{1}\right)-\ell(w)$ and $L\left(w u_{2}\right)=L\left(u_{2}\right)-\ell(w)$. Then

$$
S^{\prime}\left(w u_{1}\right)\left(S^{\prime}\left(u_{1}\right)\right)^{-1}=S^{\prime}\left(w u_{2}\right)\left(S^{\prime}\left(u_{2}\right)\right)^{-1} .
$$

Proof. We use induction on $\ell(w)$. Assume we know the result for $v$ and $w$, and that $\ell(v w)=\ell(v)+\ell(w)$. Let $u_{1}$ and $u_{2}$ be as in the proposition, for $v w$ instead of $w$. For $i=1,2$ we have $L\left(v w u_{i}\right) \geq L\left(w u_{i}\right)-\ell(v) \geq L\left(u_{i}\right)-\ell(w)-\ell(v)$ (by Lemma A.1.2). As the two extreme terms are equal by assumption, we must have $L\left(v w u_{i}\right)=L\left(w u_{i}\right)-\ell(v)$ and $L\left(w u_{i}\right)=L\left(u_{i}\right)-\ell(w)$. Applying the result for $v$, $w u_{1}, w u_{2}$ and $w, u_{1}, u_{2}$ we obtain the result for $v w, u_{1}, u_{2}$. Hence we only have to prove the proposition for $w$ of length 0 or 1 . We also only have to prove it for $u_{i} \in W$ (use relation (2) and the definition of $S^{\prime}$ ). Without loss of generality, we can assume $R$ is irreducible ( $\hat{B}$ is the product of the subgroups corresponding to each irreducible component of $R$ ).

(i) First, consider the easiest case $w=s \in S$. For $i=1,2$ we have by definition $d\left(u_{i}^{-1} A_{0}, u_{i}^{-1} s A_{0}\right)=-1$. Hence, if $s=s_{\alpha}, u_{i}^{-1}(\alpha) \in R^{+}$. Then $\ell\left(s u_{i}\right)=\ell\left(u_{i}\right)+1$ (use the criterion provided by $[\mathrm{Hu}, 1.6,1.7]$ ). Hence $S^{\prime}\left(s u_{i}\right)=$ $C^{\prime}\left(s u_{i}\right)=C^{\prime}(s) C^{\prime}\left(u_{i}\right)=S^{\prime}(s) S^{\prime}\left(u_{i}\right)$. This proves the result in this case. 
(ii) Next, assume $w$ is in $S_{\text {aff }}-S$. Then $w=t_{\gamma} s_{\gamma}$ for $\gamma$ the highest short root of $R$. We have to show that

$$
S^{\prime}(w u)\left(S^{\prime}(u)\right)^{-1}:=C^{\prime}\left(s_{\gamma} u\right) \hat{\theta}_{-u^{-1}(\gamma)} C^{\prime}(u)^{-1}
$$

does not depend on the choice of $u \in W$ such that $d\left(u^{-1} A_{0}, u^{-1} s_{\gamma} A_{0}+u^{-1}(\gamma)\right)=$ -1 . This condition amounts to requiring $u^{-1}(\gamma) \in R^{-}$. In particular, $w_{0}$ fits (recall that $w_{0}$ denotes the longest element of $\left.W\right)$. By descending induction on $l(u)$, we will show that $C^{\prime}\left(s_{\gamma} u\right) \hat{\theta}_{-u^{-1}(\gamma)} C^{\prime}(u)^{-1}=C^{\prime}\left(s_{\gamma} w_{0}\right) \hat{\theta}_{-w_{0}(\gamma)} C^{\prime}\left(w_{0}\right)^{-1}$ for any $u \in W$ such that $u^{-1}(\gamma) \in R^{-}$.

Assume $u \neq w_{0}$. Then choose $\beta \in \Phi$ such that $\ell\left(u s_{\beta}\right)=\ell(u)+1$, i.e., $u(\beta) \in R^{+}$. Then $\beta \neq-u^{-1}(\gamma)$, hence $s_{\beta} u^{-1}(\gamma) \in R^{-}$, so that we can apply the induction hypothesis to $u s_{\beta}$. Moreover,

$$
C^{\prime}\left(s_{\gamma} u s_{\beta}\right) \hat{\theta}_{-s_{\beta} u^{-1}(\gamma)} C^{\prime}\left(u s_{\beta}\right)^{-1}=C^{\prime}\left(s_{\gamma} u s_{\beta}\right) \hat{\theta}_{-s_{\beta} u^{-1}(\gamma)}\left(\hat{T}_{\beta}\right)^{-1} C^{\prime}(u)^{-1} .
$$

As $\gamma$ is a short root and a dominant weight, and $u(\beta)$ is a positive root, $\left\langle\gamma, u(\beta)^{\vee}\right\rangle=$ $\left\langle u^{-1}(\gamma), \beta^{\vee}\right\rangle$ is 0 or 1 . First, assume it is 0 . Then $s_{\beta} u^{-1}(\gamma)=u^{-1}(\gamma)$, and by relation (3) we have $\hat{\theta}_{-u^{-1}(\gamma)} \hat{T}_{\beta}^{-1}=\hat{T}_{\beta}^{-1} \hat{\theta}_{-u^{-1}(\gamma)}$. Moreover, $s_{\gamma} u(\beta)=u(\beta) \in R^{+}$, hence $\ell\left(s_{\gamma} u s_{\beta}\right)=\ell\left(s_{\gamma} u\right)+1$, and then $C^{\prime}\left(s_{\gamma} u s_{\beta}\right)=C^{\prime}\left(s_{\gamma} u\right) \hat{T}_{\beta}$. This concludes the proof in this case.

Now assume $\left\langle\gamma, u(\beta)^{\vee}\right\rangle=1$. Then $s_{\beta} u^{-1}(\gamma)=u^{-1}(\gamma)-\beta$, and by relation (4) we have $\hat{\theta}_{-s_{\beta} u^{-1}(\gamma)}=\hat{T}_{\beta} \hat{\theta}_{-u^{-1}(\gamma)} \hat{T}_{\beta}$. Moreover, $s_{\gamma} u(\beta) \in R^{-}\left(\operatorname{as}\left\langle u(\beta), \gamma^{\vee}\right\rangle>0\right)$, hence $\ell\left(s_{\gamma} u s_{\beta}\right)=\ell\left(s_{\gamma} u\right)-1$. One concludes as before.

(iii) Finally, consider some $w$ with $\ell(w)=0$. Write $w=w_{f} t_{\lambda}$. Using formula (1.1.1) we have $\left\langle\lambda, \alpha^{\vee}\right\rangle=0$ if $w_{f}(\alpha) \in R^{+}$, and $\left\langle\lambda, \alpha^{\vee}\right\rangle=-1$ if $w_{f}(\alpha) \in R^{-}$. There is no condition on $u$ in this case. Hence we have to prove that $S^{\prime}(w u)\left(S^{\prime}(u)\right)^{-1}=$ $S^{\prime}(w)$ for any $u \in W$. We will prove it by (ascending) induction on $\ell(u)$. If $u \neq \operatorname{Id}$, let $\beta \in \Phi$ and $v \in W$ be such that $u=v s_{\beta}$, with $l(v)=l(u)-1$. Then $v(\beta) \in R^{+}$. We have $S^{\prime}(w u)\left(S^{\prime}(u)\right)^{-1}=C^{\prime}\left(w_{f} v s_{\beta}\right) \hat{\theta}_{s_{\beta} v^{-1}(\lambda)}\left(\hat{T}_{\beta}\right)^{-1} C^{\prime}(v)^{-1}$.

First, assume $\ell\left(w_{f} v s_{\beta}\right)=\ell\left(w_{f} v\right)+1$, i.e., $C^{\prime}\left(w_{f} v s_{\beta}\right)=C^{\prime}\left(w_{f} v\right) \hat{T}_{\beta}$. Then $w_{f} v(\beta) \in R^{+}$. Hence $\left\langle\lambda, v(\beta)^{\vee}\right\rangle=0=\left\langle v^{-1}(\lambda), \beta^{\vee}\right\rangle$. Hence $s_{\beta} v^{-1}(\lambda)=v^{-1}(\lambda)$, and relation (3) gives $\hat{T}_{\beta} \hat{\theta}_{v^{-1}(\lambda)}=\hat{\theta}_{v^{-1}(\lambda)} \hat{T}_{\beta}$. Then the result for $u$ follows from the result for $v$.

Next, assume $\ell\left(w_{f} v s_{\beta}\right)=\ell\left(w_{f} v\right)-1$, i.e., $C^{\prime}\left(w_{f} v s_{\beta}\right)=C^{\prime}\left(w_{f} v\right)\left(\hat{T}_{\beta}\right)^{-1}$. Then $w_{f} v(\beta) \in R^{-}$. Hence $\left\langle v^{-1}(\lambda), \beta^{\vee}\right\rangle=-1$. And the result for $u$ follows from the result for $v$ and relation (4) applied to $s_{\beta} v^{-1}(\lambda)$.

A.4. End of the proof. We define a group morphism $\phi: B_{\text {aff }}^{\prime} \rightarrow \hat{B}$ by setting, for any $w \in W_{\text {aff }}^{\prime}, \phi(C(w))=S^{\prime}(w u)\left(S^{\prime}(u)\right)^{-1}$ for some $u \in W_{\text {aff }}^{\prime}$ such that $L(w u)=$ $L(u)-\ell(w)$ (such a $u$ exists by Lemma A.1.2, and this does not depend on the choice of $u$, due to Proposition A.3.1). We have already proved that these elements satisfy the relations of the definition of $B_{\text {aff }}^{\prime}$ in the beginning of the proof of Proposition A.3.1

Recall that $\psi: \hat{B} \rightarrow B_{\text {aff }}^{\prime}$ denotes the canonical morphism. It follows from Lemma A.2.1 and the diagram at the beginning of A.3 that $\psi \circ \phi=$ Id. If $s \in S$ then $L(s)=-\ell(s)$, hence one may take $u=1$. Thus $\phi \circ \psi\left(\hat{T}_{s}\right)=\phi\left(T_{s}\right)=\hat{T}_{s}$. Similarly, if $x \in \mathbb{X}$ is dominant then $L\left(t_{x}\right)=-\ell\left(t_{x}\right)$. Hence $\phi \circ \psi\left(\hat{\theta}_{x}\right)=\phi\left(\theta_{x}\right)=\phi\left(C\left(t_{x}\right)\right)=\hat{\theta}_{x}$. As these elements generate $\hat{B}$ (use relation (2)), we conclude that $\phi \circ \psi=$ Id. This concludes the proof of Theorem 1.1.3. 


\section{REFERENCES}

[SGA6] P. Berthelot, A. Grothendieck, L. Illusie, SGA 6, Théorie des intersections et théorème de Riemann-Roch, Lecture Notes in Math. 225, Springer, 1971. MR0354655 (50:7133)

[B1] R. Bezrukavnikov, Perverse coherent sheaves (after Deligne), preprint on arXiv: AG/0005152v1.

[B2] R. Bezrukavnikov, Noncommutative counterparts of the Springer resolution, Proc. I.C.M., Madrid, Spain, 2006, vol. 2, 1119-1144. MR2275638

[BMR] R. Bezrukavnikov, I. Mirković, D. Rumynin, Localization of modules for a semisimple Lie algebra in prime characteristic, with an appendix by R. Bezrukavnikov and S. Riche, preprint on arXiv: RT/0205144v8, to appear in Ann. of Math.

[BMR2] R. Bezrukavnikov, I. Mirković, D. Rumynin, Singular localization and intertwining functors for semisimple Lie algebras in prime characteristic, Nagoya Math. J. 184 (2006), 1-55. MR2285230(2007k:17024)

[Bo] A. Borel et al., Algebraic D-modules, Academic Press, 1987. MR882000 (89g:32014)

[BK] M. Brion, S. Kumar, Frobenius splitting methods in geometry and representation theory, Birkhäuser, 2004. MR2107324 (2005k:14104)

[BH] W. Bruns, J. Herzog, Cohen-Macaulay rings, Cambridge University Press, 1993. MR:1251956 (95h:13020)

[BV] W. Bruns, U. Vetter, Determinantal rings, Lecture Notes in Math. 1327, Springer, 1988. MR953963 (89i:13001)

[Ca] A. Căldăraru, The Mukai pairing, I: the Hochschild structure, preprint on arXiv: AG/0308079v2.

[CG] N. Chriss, V. Ginzburg, Representation theory and complex geometry, Birkhäuser, 1997. MR1433132(98i:22021)

[G1] V. Ginzburg, $\mathfrak{G}$-modules, Springer's representations and bivariant Chern classes, Adv. Math. 61 (1986), 1-48. MR847727 (87k:17014)

[G2] V. Ginzburg, Lagrangian construction of representations of Hecke algebras, Adv. Math. 63 (1987), 100-112. MR871082 (88e:22022)

[EGA I] A. Grothendieck, EGA I, avec la collaboration de J. Dieudonné, Springer, 1971.

[EGA III ${ }_{1}$ ] A. Grothendieck, EGA III , Étude cohomologique des faisceaux cohérents, avec la collaboration de J. Dieudonné, Publ. Math. Inst. Hautes Études Sci. 11 (1961).

[GW] S. Gukov, E. Witten, Gauge theory, ramification, and the geometric Langlands program, preprint on arXiv: hep-th/0612073v1.

[H1] R. Hartshorne, Residues and duality, Lecture Notes in Math. 20, Springer, 1966. MR0222093 (36:5145)

[H2] R. Hartshorne, Algebraic geometry, Springer, 1977. MR0463157 (57:3116)

[Hu] J. E. Humphreys, Reflection groups and Coxeter groups, Cambridge Univ. Press, 1990. MR $1066460(92 \mathrm{~h}: 20002)$

[IM] N. Iwahori, H. Matsumoto, On some Bruhat decomposition and the structure of Hecke rings of p-adic Chevalley groups, Publ. Math. Inst. Hautes Études Sci. 25 (1965), 5-48. MR0185016 (32:2486)

[KT] M. Khovanov, R. Thomas, Braid cobordisms, triangulated categories, and flag varieties, preprint on arXiv: QA/0609335v1.

[L1] G. Lusztig, Hecke algebras and Jantzen's generic decomposition patterns, Adv. Math. 37, 2 (1980), 121-164. MR591724 (82b:20059)

[L2] G. Lusztig, Affine Hecke algebras and their graded version, J. Amer. Math. Soc. 2 (1989), 599-635. MR991016 (90e:16049)

[L3] G. Lusztig, Bases in equivariant K-theory I, Represent. Theory 2 (1998), 298-369. MR:1637973 (99i:19005)

[L4] G. Lusztig, Bases in equivariant K-theory II, Represent. Theory 3 (1999), 281-353. MR 1714628 (2000h:20085)

[L5] G. Lusztig, Notes on affine Hecke algebras, in Iwahori-Hecke algebras and their representation theory, 71-103, Lecture Notes in Math. 1804, Springer, 2002. MR1979925 (2004d:20006)

[M] I. G. Macdonald, Affine Hecke algebras and orthogonal polynomials, Cambridge Univ. Press, 2003. MR1976581 (2005b:33021) 
[Ma] H. Matsumura, Commutative algebra, second edition, Benjamin, 1980. MR575344 (82i:13003)

[MR] I. Mirković, D. Rumynin, Centers of reduced enveloping algebras, Math. Z. 231 (1999), 123-132. MR 1696760 (2000i:17032)

[R] S. Riche, Koszul duality and modular representations of semi-simple Lie algebras, in preparation.

[Sp] T. A. Springer, Linear algebraic groups, Second edition, Birkhäuser, 1998. MR:1642713(99h:20075)

Université Pierre et Marie Curie, Institut de Mathématiques de Jussieu (UMR 7586 du CNRS), Équipe d'Analyse Algébrique, 175, rue du Chevaleret, 75013 Paris, France

E-mail address: riche@math.jussieu.fr

Massachusetts Institute of Technology, Cambridge, Massachusetts

E-mail address: bezrukav@math.mit.edu 